\title{
An Enhanced Inverse Finite Element Method for Displacement and Stress Monitoring of Multilayered Composite and Sandwich Structures
}

\author{
Adnan Kefal ${ }^{1,2}$, Alexander Tessler ${ }^{3}$, and Erkan Oterkus ${ }^{1}$ \\ ${ }^{1}$ Department of Naval Architecture, Ocean and Marine Engineering, University of \\ Strathclyde, 100 Montrose Street Glasgow G4 OLZ, United Kingdom \\ ${ }^{2}$ Composite Technologies Center of Excellence, Istanbul Technology Development \\ Zone, Sabanci University-Kordsa Global, Pendik, Istanbul, Turkey \\ ${ }^{3}$ Structural Mechanics and Concepts Branch, NASA Langley Research Center, Mail \\ Stop 190, Hampton, Virginia, 23681-2199, USA \\ E-mail addresses: adnan.kefal@strath.ac.uk (A. Kefal), alexander.tessler- \\ 1@nasa.gov (A. Tessler), erkan.oterkus@strath.ac.uk (E. Oterkus)
}

\begin{abstract}
The inverse finite element method (iFEM) is an innovative framework for dynamic tracking of full-field structural displacements and stresses in structures that are instrumented with a network of strain sensors. In this study, an improved iFEM formulation is proposed for displacement and stress monitoring of laminated composite and sandwich plates and shells. The formulation includes the kinematics of Refined Zigzag Theory (RZT) as its baseline. The present iFEM methodology minimizes a weighted-least-squares functional that uses the complete set of strain measures of RZT. The main advantage of the current formulation is that highly accurate through-the-thickness distributions of displacements, strains, and stresses are attainable using an element based on simple $\mathrm{C}^{0}$-continuous displacement interpolation functions. Moreover, a relatively small number of strain gauges is required. A threenode inverse-shell element, named i3-RZT, is developed. Two example problems are examined in detail: (1) a simply supported rectangular laminated composite plate and (2) a wedge structure with a hole near one of the clamped ends. The numerical results demonstrate the superior capability and potential applicability of the i3-RZT/iFEM methodology for performing accurate shape and stress sensing of complex composite structures.
\end{abstract}

Keywords: Shape sensing, stress sensing, multilayered composite structures, sandwich structures, inverse finite element method, refined zigzag theory, strain sensors. 


\section{Introduction}

Structural health monitoring (SHM) is a multidisciplinary technology that provides a conclusive real-time information regarding global and/or local structural state of a structure utilizing onboard sensing systems. The main objective of SHM is to monitor structural behavior and potentially identify damage and failure conditions. Application of SHM serves to increase human and environmental safety as well as reduce maintenance cost. Thus, SHM systems are essential technologies for many types of aeronautical, naval, and civil structures [1].

Over the last several decades, composite and sandwich material systems have been used extensively as primary structures in many different engineering applications, such as ship and offshore structures, civil and military aircraft, and wind turbines [2-4]. Such composite materials are appealing because they have superior tensile strength and resistance to compression (as a result of its fibrous nature), lighter weight, higher operating temperatures, greater stiffness, and higher reliability. Although composite structures offer numerous advantages, their load carrying capabilities can diminish due to various types of failures, such as delamination [5], fibre/matrix cracking [6], and face/core debonding [7], leading to severe reduction in their strength and stuructural integrity. Inspection of composite parts is generally cumbersome and expensive [8]. Thus, monitoring structural integrity by the way of SHM may result in improvement in safety and reduction in cost.

Shape sensing is a promising technology for the design of smart structures such as a morphing wing [9] where actuation and control systems often require real-time reconstruction of the deformations. Hence, a viable shape-sensing algorithm is necessary for the development of novel smart structures made of composite materials. Furthermore, since most of the composite and sandwich structures are built up layer by layer, they are inherently suitable for embedded optical-fiber networks, e.g., fiber Bragg grating (FBG) sensors. Such systems permit a large amount of strain data to be collected under operational conditions. As an example, Lee et al. [10] embedded FBG sensors inside a subscale composite wing to measure dynamic strains of the wing during real-time wind tunnel testing. Then, Dawood et al. [11] achieved significant 
technological improvements to embed FBG sensors within sandwich structures during their manufacturing process.

A key technology of the SHM process is real-time reconstruction of a structure's threedimensional displacement and stress fields using a network of in situ strain sensors and measured strains, which is commonly referred to as "shape and stress sensing" or “displacement and stress monitoring”. A well-suited algorithm for performing shape and stress sensing of a structure should have the following characteristics: (1) it has to be general enough to take complicated structural topologies and boundary conditions into account; (2) powerful, steady, and precise under a wide range of loading conditions, material systems, and inherent errors in the strain measurements; and finally, (3) sufficiently quick for real-time applications [12].

Tikhonov and Arsenin [13] introduced a regularization term that guarantees a confident smoothness degree to solve this inverse problem (shape sensing), and most of the inverse methods use similar type of Tikhonov's regularization [14-17]. Many shape-sensing studies have been performed to solve the problem of beam bending [1821]. In addition to the studies concerning the shape sensing of beam-type structures, the real-time monitoring of plate structures has been considered by several authors [22-25]. Most of the inverse methods mentioned above do not take into account the complexity of boundary conditions and structural topology. They also require satisfactorily precise loading information that is difficult to obtain in real time conditions outside the laboratory environment. Hence, they are not generally suited for use in on-board SHM algorithms.

The inverse finite element method (iFEM) is a state-of-the-art methodology originally introduced by Tessler and Spangler [26, 27] for real-time reconstruction of full-field structural displacements in plate and shell structures that are instrumented by strain sensors. The general mathematical concept of the iFEM methodology uses a leastsquares variational principle that minimizes the sum of squared errors between the analytical and experimental values of strain measures. As opposed to other developed SHM methods, the iFEM methodology is a revolutionary shape- and stress-sensing algorithm and possesses the aforementioned characteristics required for a powerful SHM algorithm. The main advantage of the iFEM algorithm is that static and dynamic 
behavior of any structure can be obtained without prior knowledge of loading. Since the first publication of the iFEM algorithm in [26, 27], various theoretical studies including a three-node inverse shell element [28], a four-node quadrilateral inverseshell element [29], and an efficient inverse frame element [12, 30-32] have been devoted to expanding the horizon of the iFEM methodology in the literature. In addition, the iFEM framework was applied to not only SHM of future aerospace vehicles in [33-35, 56], but also shape and stress sensing of marine structures in [3638]. Recently, a U.S. patent (US 8,515,675 B2) was obtained for a system that performs shape sensing of a downhole structure by using the iFEM methodology [39]. All investigations have so far demonstrated that the iFEM methodology is a superior shape- and stress-sensing algorithm.

Most of the iFEM-based shape-sensing case studies described in the previous two paragraphs adopted first-order shear deformation theory (FSDT) [40, 41]. Even though commonly known as a precise theory, FSDT may result in slightly insufficient estimates when used for relatively thick composite and/or sandwich plates. For such plates, an accurate formulation is required that takes into consideration the discrete nature of individual laminae as well as the varying stiffness properties of the core. Tessler et al. [42, 43] developed such a formulation and called it the Refined Zigzag Theory (RZT). Recently, Cerracchio et al. [44, 45] improved the original iFEM formulation [26, 27] by adding the kinematic assumptions of the RZT. This recent formulation aimed to deal with displacement and stress monitoring of multilayered composite and sandwich structures that possesses a high degree of anisotropy and heterogeneity. Although their formulation performed well for sandwich plates, the proposed variational statement does not involve contributions of average (first) transverse-shear strain measures and accommodates only the second transverse-shear strain measures of RZT in addition to the membrane, bending, and zigzag contributions. Moreover, they have developed a three-node inverse-plate element, called iRZT3, which can only be used for plate structures. Furthermore, the authors utilized a least-squares functional without the weighting coefficients, thus iRZT3 element cannot be used to analyze problems that involve relatively few strain gauges (i.e., sparse in situ strain data). 
In the current study, an enhanced iFEM formulation is introduced to address the aforementioned shortcomings of the RZT-based iFEM formulation [44, 45]. The leastsquares functional of the improved iFEM formulation is defined using the complete set of strain measures consistent with the RZT plate theory. These strain measures involve membrane strain measures, bending curvatures, zigzag strain measures, and full (first and second) transverse-shear strain measures of the RZT. The present iFEM methodology is applicable for the analysis of thin and moderately thick plate and shell structures manufactured using composite materials. To increase the practical usefulness of the present iFEM methodology, weighting constants that were originally introduced by Tessler et al. [46] are utilized to define the least-squares functional of the current iFEM formulation. The improved iFEM formulation is well-suited for $\mathrm{C}^{0}$ continuous displacement interpolation functions, i.e., standard polynomial-based functions, thus a robust and computationally efficient three-node inverse-shell element, i3-RZT, is developed for performing accurate shape and stress sensing of complex shell structures.

In the remainder of the paper, the theoretical foundation of the current formulation and its quantitative assessment are detailed. In section 2, the kinematics of the RZT plate theory are briefly reviewed and the strain field is properly rewritten in order to define the strain measures to be used in the iFEM weighted-least-squares functional. Then, the computation of experimentally measured (in situ) section strains is described by introducing a computational tool that can be used to obtain the continuous form of the in situ section strains and to calculate experimental transverse-shear strain measures. Afterwards, the variational statement of the present iFEM methodology is introduced for both discrete and continuous forms of the in situ section strains. In section 3, the numerical implementation of the i3-RZT element is described based on the improved iFEM formulation. In section 4, three different simply supported rectangular laminates, each of which has a different laminate stacking sequence (uniaxial, crossply and angle-ply), are analyzed as a benchmark problem. Then, the applicability of the improved iFEM formulation to more complex composite/sandwich structures is demonstrated by analyzing a wedge structure with a hole near one of the clamped ends. Finally, several conclusions emphasizing the benefits of the improved iFEM methodology are highlighted in section 5. 


\section{The Enhanced iFEM Formulation for Composite Plate and Shell Structures}

\subsection{The inverse problem}

Consider a plate (or laminate) with thickness of $2 h$ that is consisted of $N$ perfectly bonded orthotropic layers (or laminae) as depicted in Figure 1. The laminate is oriented with respect to an orthogonal Cartesian coordinate system where the symbol identifies the through-the-thickness coordinate with $z=0$ referring to as the reference plane (or mid-plane) of the plate. The symbols $\left(x_{1}, x_{2}\right) \in A$ represent the in-plane coordinates, where $A$ denotes the area of the mid-plane (refer to Figure 1).

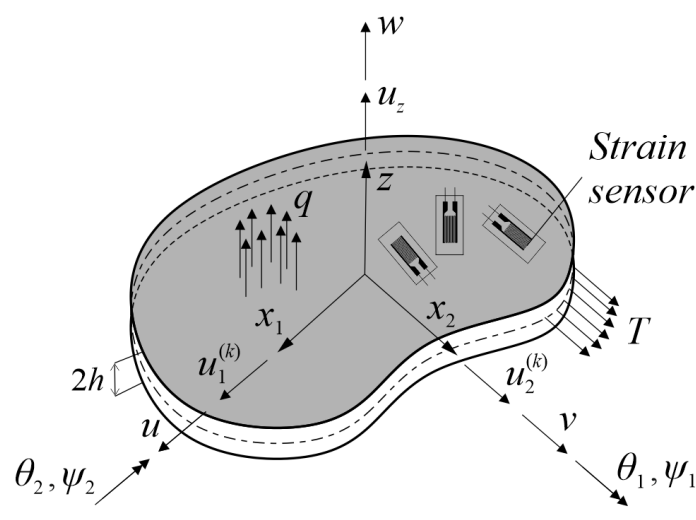

(a)

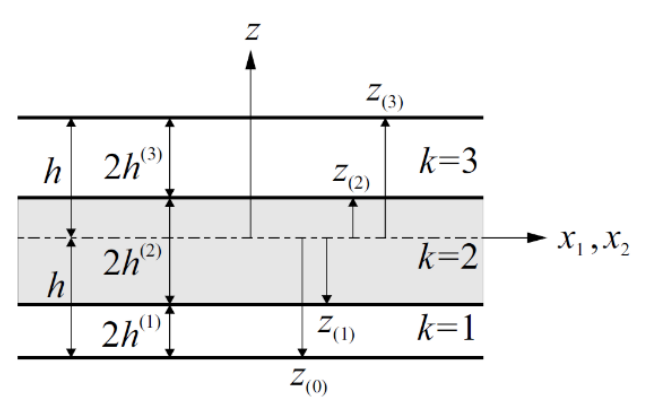

(b)

Figure 1. (a) RZT-based iFEM plate notation; (b) Layer notation for a three-layer laminate.

In remainder of this paper, if not otherwise specified, the superscript $(k)$ is used to indicate the $k$-th lamina, whereas the subscript $(k)$ defines the interface between the $k$ th and ( $k+1)$-th laminae. As shown in Figure 1(b), the $k$-th lamina thickness is therefore defined in the range $z \in\left[z_{(k-1)}, z_{(k)}\right](k=1-N)$. The laminate is constrained against the rigid body motion and subjected to external loads including the planar and throughthe-thickness direction components ( $q$ and $T$ ). The strain sensors are mounted at discrete locations on top and bottom surfaces of the laminate and also embedded inside the laminate (e.g., $j$-th interface located at $z_{(j)}$ ), supplying real-time strain measurements. Herein the inverse problem is shape and stress sensing of the presented 
laminate, which will be solved by an enhanced iFEM methodology that is based on kinematics of the RZT and utilizes only the in situ discrete surface strains and boundary restraints for the solution.

\subsection{Kinematic relations}

According to RZT [43], the orthogonal components of the displacement vector, corresponding to material points of the laminate (refer to Figure 1), can be expressed as

$$
\begin{aligned}
& u_{1}^{(k)}\left(x_{1}, x_{2}, z\right) \equiv u\left(x_{1}, x_{2}\right)+z \theta_{1}\left(x_{1}, x_{2}\right)+\phi_{1}^{(k)}(z) \psi_{1}\left(x_{1}, x_{2}\right) \\
& u_{2}^{(k)}\left(x_{1}, x_{2}, z\right) \equiv v\left(x_{1}, x_{2}\right)+z \theta_{2}\left(x_{1}, x_{2}\right)+\phi_{2}^{(k)}(z) \psi_{2}\left(x_{1}, x_{2}\right) \\
& u_{z}\left(x_{1}, x_{2}, z\right) \equiv w\left(x_{1}, x_{2}\right)
\end{aligned}
$$

where the in-plane displacement components $u_{\alpha}^{(k)} \equiv u_{\alpha}^{(k)}\left(x_{1}, x_{2}, z\right)(\alpha=1,2)$ contain constant, linear, and zigzag variations through-the-thickness coordinate. The zigzag variations are $\mathrm{C}^{0}$-continuous functions with discontinuous thickness-direction derivatives along the lamina interfaces. In Eq. (1c), the transverse displacement $u_{z} \equiv u_{z}\left(x_{1}, x_{2}, z\right)$ is assumed to be constant through the thickness and is independent of constitutive properties of the $k$-th lamina; hence, the superscript $(k)$ does not appear in its definition and the function $w \equiv w\left(x_{1}, x_{2}\right)$ represents the transverse deflection of the laminate.

In Eqs. (1a-b), the constant translations along $x_{1}$ and $x_{2}$ directions are denoted by the functions $u \equiv u\left(x_{1}, x_{2}\right)$ and $v \equiv v\left(x_{1}, x_{2}\right)$, respectively. Moreover, bending rotations around the positive $x_{2}$ and negative $x_{1}$ directions are represented as $\theta_{1} \equiv \theta_{1}\left(x_{1}, x_{2}\right)$ and $\theta_{2} \equiv \theta_{2}\left(x_{1}, x_{2}\right)$, respectively. Furthermore, the functions $\phi_{1}^{(k)} \equiv \phi_{1}^{(k)}(z)$ and $\phi_{2}^{(k)} \equiv \phi_{2}^{(k)}(z) \quad$ denote through-the-thickness piecewise-linear zigzag functions associated with heterogeneous plates. Finally, the functions $\psi_{1} \equiv \psi_{1}\left(x_{1}, x_{2}\right)$ and $\psi_{2} \equiv \psi_{2}\left(x_{1}, x_{2}\right)$ represent the spatial amplitudes of the zigzag displacements, and they are the unknowns in the analysis together with the other five kinematic variables. These kinematic variables can be expressed by a compact vector form as 


$$
\mathbf{u}=\left[\begin{array}{lllllll}
u & v & w & \theta_{1} & \theta_{2} & \psi_{1} & \psi_{2}
\end{array}\right]^{T}
$$

Following the approach proposed in [43], the zigzag functions $\phi_{\alpha}^{(k)}(\alpha=1,2)$ can be defined as

$$
\begin{aligned}
\phi_{1}^{(k)} & \equiv \frac{1}{2}\left(1-\xi^{(k)}\right) u_{(k-1)}+\frac{1}{2}\left(1+\xi^{(k)}\right) u_{(k)} \\
\phi_{2}^{(k)} & \equiv \frac{1}{2}\left(1-\xi^{(k)}\right) v_{(k-1)}+\frac{1}{2}\left(1+\xi^{(k)}\right) v_{(k)}
\end{aligned}
$$

with

$$
\xi^{(k)}=\left[\frac{z-z_{(k-1)}}{h^{(k)}}-1\right] \in[-1,+1] \quad(k=1-N)
$$

where the first lamina beginning at $z_{(0)}=-h$, the last $(N$-th) lamina ending at $z_{(N)}=+h$, and the $k$-th lamina ending at $z_{(k)}=z_{(k-1)}+2 h^{(k)}$ where $2 h^{(k)}$ denotes the thickness of the $k$-th lamina. Evaluating Eqs. (3a-b) at the lamina interfaces gives rise to the definitions of the interfacial displacements

$$
\begin{array}{ll}
u_{(k-1)}=\phi_{1}^{(k)}\left(\xi^{(k)}=-1\right), & u_{(k)}=\phi_{1}^{(k)}\left(\xi^{(k)}=+1\right) \\
v_{(k-1)}=\phi_{2}^{(k)}\left(\xi^{(k)}=-1\right), & v_{(k)}=\phi_{2}^{(k)}\left(\xi^{(k)}=+1\right) \quad(k=1-N)
\end{array}
$$

where the interfacial displacements at the bottom and top plate surfaces vanish identically; that is,

$$
u_{(0)}=u_{(N)}=v_{(0)}=v_{(N)}=0
$$

According to Tessler and co-workers [43], the $u_{(k)}$ and $v_{(k)}$ interfacial values of the zigzag functions are expressed in terms of piecewise constant slope functions $\beta_{\alpha}^{(k)}(\alpha=1,2 ; k=1-N)$ as

$$
\left\{\begin{array}{l}
u_{(k)} \\
v_{(k)}
\end{array}\right\}=2 h^{(k)}\left\{\begin{array}{l}
\beta_{1}^{(k)} \\
\beta_{2}^{(k)}
\end{array}\right\}+\left\{\begin{array}{l}
u_{(k-1)} \\
v_{(k-1)}
\end{array}\right\}(k=1-N)
$$


where the $\beta_{\alpha}^{(k)}(\alpha=1,2)$ slope of the zigzag functions, namely derivatives of zigzag functions with respect to the through-the-thickness coordinate $z$, can be explicitly defined for the $k$-th layer as

$$
\left\{\begin{array}{c}
\beta_{1}^{(k)} \\
\beta_{2}^{(k)}
\end{array}\right\}=\left\{\begin{array}{l}
G_{1} / Q_{11}^{(k)}-1 \\
G_{2} / Q_{22}^{(k)}-1
\end{array}\right\} \quad(k=1-N)
$$

with

$$
\left\{\begin{array}{l}
G_{1} \\
G_{2}
\end{array}\right\}=\left\{\begin{array}{l}
\left(\frac{1}{h} \sum_{i=1}^{N} \frac{h^{(i)}}{Q_{11}^{(i)}}\right)^{-1} \\
\left(\frac{1}{h} \sum_{i=1}^{N} \frac{h^{(i)}}{Q_{22}^{(i)}}\right)^{-1}
\end{array}\right\}
$$

where $G_{1}$ and $G_{2}$ are the weighted-average transverse-shear stiffness coefficients of their respective lamina-level coefficients, $Q_{11}^{(k)}$ and $Q_{22}^{(k)}(k=1-N)$.

According to the strain-displacement relationship of the linear elasticity theory, the inplane strain components can be defined as

$$
\begin{aligned}
& \varepsilon_{11}^{(k)}=u_{1,1}^{(k)}=e_{1}+z \kappa_{4}+\mu_{7}^{(k)} \\
& \varepsilon_{22}^{(k)}=u_{2,2}^{(k)}=e_{2}+z \kappa_{5}+\mu_{8}^{(k)} \\
& \gamma_{12}^{(k)}=u_{1,2}^{(k)}+u_{2,1}^{(k)}=e_{3}+z \kappa_{6}+\mu_{9}^{(k)}
\end{aligned}
$$

where, henceforth, ()$_{, \alpha} \equiv \frac{\partial()}{\partial x_{\alpha}}$ denotes a partial derivative with respect to in-plane coordinate $x_{\alpha}(\alpha=1,2)$. In Eqs. (6a-c), the symbols $e_{\alpha}(\alpha=1-3), \kappa_{\alpha}(\alpha=4-6)$, and $\mu_{\alpha}^{(k)}(\alpha=7-9)$ denote the membrane strain measures, bending curvatures, and zigzag strain measures. The explicit forms of these terms can be defined in terms of the compact form of kinematic variables $\mathbf{u}$ as

$$
\mathbf{e}(\mathbf{u}) \equiv\left\{\begin{array}{c}
e_{1} \\
e_{2} \\
e_{3}
\end{array}\right\}=\left\{\begin{array}{c}
u_{, 1} \\
v_{, 2} \\
u_{, 2}+v_{, 1}
\end{array}\right\}
$$




$$
\begin{aligned}
& \mathbf{\kappa}(\mathbf{u}) \equiv\left\{\begin{array}{l}
\kappa_{4} \\
\kappa_{5} \\
\kappa_{6}
\end{array}\right\}=\left\{\begin{array}{c}
\theta_{1,1} \\
\theta_{2,2} \\
\theta_{1,2}+\theta_{2,1}
\end{array}\right\} \\
& \boldsymbol{\mu}^{(k)}(\mathbf{u}) \equiv\left\{\begin{array}{l}
\mu_{7}^{(k)} \\
\mu_{8}^{(k)} \\
\mu_{9}^{(k)}
\end{array}\right\}=\left\{\begin{array}{c}
\phi_{1}^{(k)} \mu_{7} \\
\phi_{2}^{(k)} \mu_{8} \\
\phi_{1}^{(k)} \mu_{9}+\phi_{2}^{(k)} \mu_{10}
\end{array}\right\}
\end{aligned}
$$

with

$\left[\begin{array}{llll}\mu_{7} & \mu_{8} & \mu_{9} & \mu_{10}\end{array}\right]=\left[\begin{array}{llll}\psi_{1,1} & \psi_{2,2} & \psi_{1,2} & \psi_{2,1}\end{array}\right]$

where $\mu_{\alpha}(\alpha=7-10)$ represents the zigzag curvatures. In order to define the zigzag strain measures $\boldsymbol{\mu}^{(k)}(\mathbf{u})$, the zigzag functions $\phi_{\alpha}^{(k)}(\alpha=1,2)$ and the zigzag curvatures $\mu_{\alpha}(\alpha=7-10)$ are coupled in Eq. (7c).

The transverse-shear strain components can be defined as

$\gamma_{1 z}^{(k)}=u_{1, z}^{(k)}+u_{z, 1}=\left(1+\beta_{1}^{(k)}\right) \gamma_{10}-\beta_{1}^{(k)} \eta_{12}$

$\gamma_{2 z}^{(k)}=u_{2, z}^{(k)}+u_{z, 2}=\left(1+\beta_{2}^{(k)}\right) \gamma_{11}-\beta_{2}^{(k)} \eta_{13}$

where ()$_{, z} \equiv \frac{\partial()}{\partial z}$ denotes a partial derivative with respect to through-the-thickness coordinate $z$. In Eqs. (8a-b), the symbols $\gamma_{\alpha}(\alpha=10,11)$ and $\eta_{\alpha}(\alpha=12,13)$ denote the average (first) and second transverse-shear strain measures of the RZT, respectively. The explicit form of these terms can be described in terms of the kinematic variables $\mathbf{u}$ as

$$
\begin{gathered}
\boldsymbol{\gamma}(\mathbf{u}) \equiv\left\{\begin{array}{l}
\gamma_{10} \\
\gamma_{11}
\end{array}\right\}=\left\{\begin{array}{l}
w_{, 1}+\theta_{1} \\
w_{, 2}+\theta_{2}
\end{array}\right\} \\
\boldsymbol{\eta}(\mathbf{u}) \equiv\left\{\begin{array}{l}
\eta_{12} \\
\eta_{13}
\end{array}\right\}=\left\{\begin{array}{l}
\gamma_{10}-\psi_{1} \\
\gamma_{11}-\psi_{2}
\end{array}\right\}
\end{gathered}
$$

Integrating Eq. (8) across the laminate thickness and normalizing the result by the total laminate thickness reveal that 
$\left\{\begin{array}{l}\gamma_{10} \\ \gamma_{11}\end{array}\right\}=\frac{1}{2 h} \int_{-h}^{h}\left\{\begin{array}{l}\gamma_{1 z}^{(k)} \\ \gamma_{2 z}^{(k)}\end{array}\right\} d z$

Thus, the first transverse-shear strain measures $\gamma_{\alpha}(\alpha=10,11)$ of the RZT coincides with the shear angles of FSDT. This indicates that the zigzag rotations $\psi_{\alpha}(\alpha=1,2)$ has no contribution to the average transverse-shear strains.

\subsection{Computation of experimental section strains}

The computation of the in situ section strains is vital for performing an accurate RZTbased iFEM analysis. Conventional strain rosettes and embedded FBG sensors can be used to collect a large amount of on-board strain data. To compute in situ section strains, as depicted in Figure 2, at least three different in situ strain rosettes $\left(\boldsymbol{\varepsilon}_{i}^{+}, \boldsymbol{\varepsilon}_{i}^{-}, \boldsymbol{\varepsilon}_{i}^{j}\right)$ must be placed along the thickness direction of each particular location $\left(\mathbf{x}_{i}, z\right)(i=1-n)$ where $\mathbf{x}_{i} \equiv\left(x_{1}, x_{2}\right)_{i}$ and $z \in[-h,+h]$ are located within the laminate. Note that the symbol ' $n$ ' stands for the number of discrete locations at which experimental strain measures are calculated using the surface strain sensors.

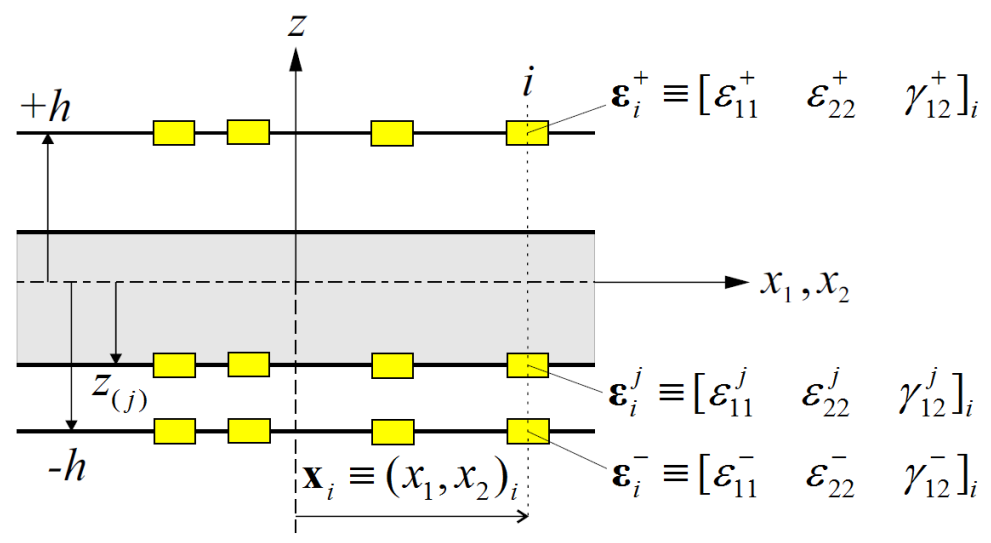

Figure 2. Strain rosettes and experimental surface strain measurements.

The exact locations of these sensors and their surface strain measurements (readings) are defined as

$$
\begin{aligned}
& \boldsymbol{\varepsilon}_{i}^{+}\left(\mathbf{x}_{i}, z_{i}=+h\right)=\left[\begin{array}{lll}
\varepsilon_{11}^{+} & \varepsilon_{22}^{+} & \gamma_{12}^{+}
\end{array}\right]_{i} \\
& \boldsymbol{\varepsilon}_{i}^{-}\left(\mathbf{x}_{i}, z_{i}=-h\right)=\left[\begin{array}{lll}
\varepsilon_{11}^{-} & \varepsilon_{22}^{-} & \gamma_{12}^{-}
\end{array}\right]_{i} \\
& \boldsymbol{\varepsilon}_{i}^{j}\left(\mathbf{x}_{i}, z_{i}=z_{(j)}\right)=\left[\begin{array}{lll}
\varepsilon_{11}^{j} & \varepsilon_{22}^{j} & \gamma_{12}^{j}
\end{array}\right]_{i}
\end{aligned}
$$


where the surface strain readings with the superscripts '+', '-', and ' $j$ ' refer to as the strain rosettes located on the top surface, bottom surface and $j$-th interface of the laminate, respectively. In Eq. (11), normal strain measurements (along $x_{1}$ and $x_{2}$ directions) and shear strain measurement (in $x_{1} x_{2}$ plane) are identified by subscripts (11), (22), and (12), respectively.

The zigzag contributions to the in-plane strains vanish at the top and bottom surfaces. Therefore, experimentally measured membrane strains and bending curvatures can be determined using the same relations of the original iFEM plate formulation [26, 27]. These in situ membrane strains $\mathbf{E}_{i}$ and bending curvatures $\mathbf{K}_{i}$ can be computed at a particular discrete location $\mathbf{x}_{i}$ as

$$
\begin{gathered}
\mathbf{E}_{i} \equiv\left\{\begin{array}{l}
\mathrm{E}_{1} \\
\mathrm{E}_{2} \\
\mathrm{E}_{3}
\end{array}\right\}_{i}=\frac{1}{2}\left\{\begin{array}{l}
\varepsilon_{11}^{+}+\varepsilon_{11}^{-} \\
\varepsilon_{22}^{+}+\varepsilon_{22}^{-} \\
\gamma_{12}^{+}+\gamma_{12}^{-}
\end{array}\right\}_{i}(i=1-n) \\
\mathbf{K}_{i} \equiv\left\{\begin{array}{l}
\mathrm{K}_{4} \\
\mathrm{~K}_{5} \\
\mathrm{~K}_{6}
\end{array}\right\}_{i}=\frac{1}{2 h}\left\{\begin{array}{l}
\varepsilon_{11}^{+}-\varepsilon_{11}^{-} \\
\varepsilon_{22}^{+}-\varepsilon_{22}^{-} \\
\gamma_{12}^{+}-\gamma_{12}^{-}
\end{array}\right\}_{i}(i=1-n)
\end{gathered}
$$

where in situ section strains, $\mathbf{E}_{i}$ and $\mathbf{K}_{i}$, correspond to their analytic counterparts, $\mathbf{e}(\mathbf{u})$ and $\boldsymbol{\kappa}(\mathbf{u})$, given by Eqs. (7a-b), respectively. Substituting $j$-th interface strain readings $\left(\varepsilon_{11}^{j}, \varepsilon_{22}^{j}, \gamma_{12}^{j}\right)_{i}$ and in situ section strains $\mathbf{E}_{i}$ and $\mathbf{K}_{i}$ into Eq. (6), the in situ zigzag strain measures $\mathbf{M}_{i}^{j}$ can be computed at a particular discrete location $\left(\mathbf{x}_{i}, z_{i}=z_{(j)}\right)$ as $[44,45]$

$$
\mathbf{M}_{i}^{j} \equiv\left\{\begin{array}{l}
\mathbf{M}_{7}^{j} \\
\mathbf{M}_{8}^{j} \\
\mathbf{M}_{9}^{j}
\end{array}\right\}_{i}=\left\{\begin{array}{l}
\varepsilon_{11}^{j}-\mathrm{E}_{1}-z_{(j)} \mathrm{K}_{4} \\
\varepsilon_{22}^{j}-\mathrm{E}_{2}-z_{(j)} \mathrm{K}_{5} \\
\gamma_{12}^{j}-\mathrm{E}_{3}-z_{(j)} \mathrm{K}_{6}
\end{array}\right\}_{i}(i=1-n)
$$

The in situ section strains $\mathbf{M}_{i}^{j}$ are evaluated at the $j$-th interface only; therefore, their analytic counterpart $\boldsymbol{\mu}^{(k)}(\mathbf{u})$ given by Eq. (7c) must also be computed at exactly the 
same locations $\left(\mathbf{x}_{i}, z_{i}=z_{(j)}\right)$. Moreover, the in situ transverse-shear strain measures can be represented by a compact vector form as

$\mathbf{G}_{i}=\left[\begin{array}{llll}\Gamma_{10} & \Gamma_{11} & \mathrm{H}_{12} & \mathrm{H}_{13}\end{array}\right]_{i}(i=1-n)$

where $\Gamma_{\alpha i}(\alpha=10,11)$ and $\mathrm{H}_{\alpha i}(\alpha=12,13)$ denote discrete first and second transverseshear strain measures that correspond to their analytic counterparts, $\gamma(\mathbf{u})$ and $\boldsymbol{\eta}(\mathbf{u})$, given by Eqs. (9a-b), respectively. In Eqs. (12-14), the uppercase Greek letters are used to indicate the existence of experimental error in the strain measurements.

The surface strain readings obtained from the in situ strain rosettes $\left(\boldsymbol{\varepsilon}_{i}^{+}, \boldsymbol{\varepsilon}_{i}^{-}, \boldsymbol{\varepsilon}_{i}^{j}\right)$ cannot be used straightaway to compute the $\mathbf{G}_{i}$. However, deformation of thin shells exhibits a much smaller transverse-shear strains than in-plane strains. Therefore, the iFEM analysis of thin shells can be safely performed by omitting the $\mathbf{G}_{i}$ contributions. Instead, in deformation of thick shells, a considerable amount of the transverse deflection is caused by transverse-shear stresses, hence it is necessary to compute the $\mathbf{G}_{i}$ for obtaining accurate deformed shapes of the shell.

Experimental strain data can be processed analytically by using curve fitting or smoothing techniques [47]. For example, a smoothing procedure, developed by Tessler et al. [48, 49], called smoothing element analysis (SEA), can be utilized to smooth the discrete in situ strain measures, $\mathbf{E}_{i}, \mathbf{K}_{i}$, and $\mathbf{M}_{i}^{j}$. In this regard, the nine independent section strain measures, $\mathbf{E}, \mathbf{K}$, and $\mathbf{M}^{j}$, can be obtained as $\mathrm{C}^{1}$-continuous polynomial functions (i.e., having $\mathrm{C}^{0}$-continuous first-order derivatives) that are defined everywhere in the mid-plane of the laminate. Note that, hereafter, the ' $i$ ' subscript is removed to differentiate these continuous quantities from the discrete ones.

The main advantage of the SEA is that it enables the first-order derivatives of in situ membrane strains, bending curvatures, and zigzag curvatures to be accurately computed and subsequently used to obtain the in situ transverse-shear strain measures, G. The computational tool depicted in Figure 3 schematically describes all the steps required to calculate the $\mathbf{G}$. 


\section{Experimental section strains}

1.1. Obtain surface strain measurements $\left(\boldsymbol{\varepsilon}_{i}^{+}, \boldsymbol{\varepsilon}_{i}^{-}, \boldsymbol{\varepsilon}_{i}^{j}\right)$

1.2. Compute discrete in-situ strain measures $\left(\mathbf{E}_{i}, \mathbf{K}_{i}, \mathbf{M}_{i}^{j}\right)$

II. SEA

2.1. Map $\left(\mathbf{E}_{i}, \mathbf{K}_{i}, \mathbf{M}_{i}^{j}\right)$ on to a coarse SEA mesh

2.2. Perform SEA for each strain measure

2.3. Recover accurate $C^{1}$-continuous $\left(\mathbf{E}, \mathbf{K}, \mathbf{M}^{j}\right)$ strain measures

\section{III. iFEM}

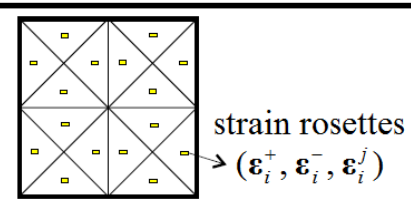

3.1. Perform a preliminary iFEM analysis using $\left(\mathbf{E}, \mathbf{K}, \mathbf{M}^{j}\right)$

3.2. Compute discrete zigzag shear curvatures $\mathrm{M}_{\alpha i}(\alpha=9,10)$

IV. SEA

4.1. Map $\mathrm{M}_{\alpha i}(\alpha=9,10)$ onto a coarse SEA mesh

4.2. Perform SEA for each curvature

4.3. Recover accurate $C^{1}$-continuous $\mathrm{M}_{\alpha}(\alpha=9,10)$ curvatures

\section{Transverse-shear strain measures}

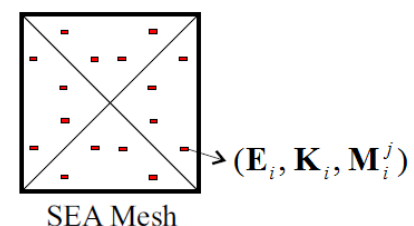

5.1. Find first order derivatives of $\mathbf{E}, \mathbf{K}$, and $\mathrm{M}_{\alpha}(\alpha=7-10)$

5.2. Solve equilibrium equations of RZT plate theory

5.3 Obtain accurate $\mathrm{C}^{0}$-continuous $\mathbf{G}$ transverse-shear strain measures

\section{VI. iFEM}

6.1. Perform a second iFEM analysis using $\left(\mathbf{E}, \mathbf{K}, \mathbf{M}^{j}, \mathbf{G}\right)$

6.2. Reconstruct the kinematic variables $\mathbf{u}$

6.3. Calculate stress components using constitutive equations

Figure 3. The iFEM computational tool to calculate all in situ section strains.

The computational method involves solving the four equilibrium equations for the transverse-shear stress resultants. For RZT, these equilibrium equations are explicitly given in [46] as

$$
\begin{aligned}
& M_{1,1}+M_{12,2}-Q_{1}=0 \\
& M_{12,1}+M_{2,2}-Q_{2}=0 \\
& M_{1,1}^{\phi}+M_{12,2}^{\phi}-Q_{1}^{\phi}=0 \\
& M_{21,1}^{\phi}+M_{2,2}^{\phi}-Q_{2}^{\phi}=0
\end{aligned}
$$


with

$\mathbf{M}_{b}=\left[\begin{array}{lllllll}M_{1} & M_{2} & M_{12} & M_{1}^{\phi} & M_{2}^{\phi} & M_{12}^{\phi} & M_{21}^{\phi}\end{array}\right]^{T}$

and

$\mathbf{Q}_{s}=\left[\begin{array}{llll}Q_{1} & Q_{2} & Q_{1}^{\phi} & Q_{2}^{\phi}\end{array}\right]^{T}$

representing the bending and transverse-shear stress resultants, respectively. These stress resultants can be explicitly defined utilizing the constitutive relations of the RZT as

$\mathbf{M}_{b}=\mathbf{A} \mathbf{E}+\mathbf{D}\left\{\begin{array}{l}\mathbf{K} \\ \mathbf{M}\end{array}\right\}$

$\mathbf{Q}_{s}=\mathbf{H G}$

where

$\mathbf{M}=\left[\begin{array}{llll}\mathbf{M}_{7} & \mathbf{M}_{8} & \mathbf{M}_{9} & \mathbf{M}_{10}\end{array}\right]^{T}$

denotes the vector containing continuous form of the in situ zigzag curvatures that correspond to their analytic counterparts, $\mu_{\alpha}(\alpha=7-10)$, given by Eq. (7d). The constitutive stiffness coefficients given in Eqs. (16a-b) can be calculated as

$$
\mathbf{A}=\int_{-h}^{+h} \mathbf{B}_{\phi}^{T} \mathbf{C} d z, \mathbf{D}=\int_{-h}^{+h} \mathbf{B}_{\phi}^{T} \mathbf{C} \mathbf{B}_{\phi} d z, \text { and } \mathbf{H}=\int_{-h}^{+h} \mathbf{B}_{\beta}^{T} \mathbf{Q} \mathbf{B}_{\gamma} d z
$$

with

$$
\begin{aligned}
\mathbf{B}_{\phi} & =\left[\begin{array}{ccccccc}
z & 0 & 0 & \phi_{1}^{(k)} & 0 & 0 & 0 \\
0 & z & 0 & 0 & \phi_{2}^{(k)} & 0 & 0 \\
0 & 0 & z & 0 & 0 & \phi_{1}^{(k)} & \phi_{2}^{(k)}
\end{array}\right], \mathbf{B}_{\beta}=\left[\begin{array}{cccc}
1 & 0 & \beta_{1}^{(k)} & 0 \\
0 & 1 & 0 & \beta_{2}^{(k)}
\end{array}\right], \\
\mathbf{B}_{\gamma} & =\left[\begin{array}{cccc}
1+\beta_{1}^{(k)} & 0 & -\beta_{1}^{(k)} & 0 \\
0 & 1+\beta_{2}^{(k)} & 0 & -\beta_{2}^{(k)}
\end{array}\right]
\end{aligned}
$$

and 
$\mathbf{C}=\left[\begin{array}{lll}C_{11} & C_{12} & C_{16} \\ C_{12} & C_{22} & C_{26} \\ C_{16} & C_{26} & C_{66}\end{array}\right]^{(k)}, \mathbf{Q}=\left[\begin{array}{ll}Q_{11} & Q_{12} \\ Q_{12} & Q_{22}\end{array}\right]^{(k)}$

where the matrices $\mathbf{C}$ and $\mathbf{Q}$ contain the in-plane and transverse-shear elastic stiffness coefficients for the $k$-th layer, respectively.

Substituting Eqs. (16a-b) into the equilibrium equations, Eqs. (15a-d), yields the transverse-shear strain measures, $\mathbf{G}$, as

$$
\mathbf{G}=\mathbf{H}^{-1}\left(\mathbf{I}_{1} \mathbf{M}_{b, 1}+\mathbf{I}_{2} \mathbf{M}_{b, 2}\right)
$$

with

$$
\mathbf{M}_{b, \alpha}=\mathbf{A} \mathbf{E}_{, \alpha}+\mathbf{D}\left\{\begin{array}{l}
\mathbf{K} \\
\mathbf{M}
\end{array}\right\}_{, \alpha} \quad(\alpha=1,2)
$$

and

$$
\mathbf{I}_{1}=\left[\begin{array}{lllllll}
1 & 0 & 0 & 0 & 0 & 0 & 0 \\
0 & 0 & 1 & 0 & 0 & 0 & 0 \\
0 & 0 & 0 & 1 & 0 & 0 & 0 \\
0 & 0 & 0 & 0 & 0 & 0 & 1
\end{array}\right], \mathbf{I}_{2}=\left[\begin{array}{lllllll}
0 & 0 & 1 & 0 & 0 & 0 & 0 \\
0 & 1 & 0 & 0 & 0 & 0 & 0 \\
0 & 0 & 0 & 0 & 0 & 1 & 0 \\
0 & 0 & 0 & 0 & 1 & 0 & 0
\end{array}\right]
$$

The $\mathrm{C}^{0}$-continuous first-order derivatives of $\mathbf{E}$ and $\mathbf{K}$ can be readily obtained to calculate Eqs. (18a-c) through the stage II. SEA shown in Figure 3. The $\mathrm{C}^{1}$-continuous form of the $\mathbf{M}$ has to be also well-defined to calculate the $\mathbf{M}_{, \alpha}(\alpha=1,2)$ terms in Eqs. (18a-c). In fact, the continuous form of $\mathrm{M}_{\alpha}(\alpha=7,8)$ can be simply obtained from the continuous form of zigzag strain measures, $\mathrm{M}_{\alpha}^{j}(\alpha=7,8)$, as

$$
\left[\begin{array}{ll}
\mathrm{M}_{7} & \mathrm{M}_{8}
\end{array}\right]=\left[\begin{array}{ll}
\frac{\mathrm{M}_{7}^{j}}{\phi_{1}^{(k)}\left(z_{(j)}\right)} & \frac{\mathrm{M}_{8}^{j}}{\phi_{2}^{(k)}\left(z_{(j)}\right)}
\end{array}\right]
$$

where $\phi_{\alpha}^{(k)}\left(z_{(j)}\right)(\alpha=1,2)$ represents the zigzag function evaluated at the $j$-th interface, $\left(z_{i}=z_{(j)}\right)$. On the other hand, as only three strain rosettes are located through the thickness of each discrete location, $\mathbf{x}_{i}$, neither the discrete form, $\mathrm{M}_{\alpha i}(\alpha=9,10)$, nor the continuous form, $\mathrm{M}_{\alpha}(\alpha=9,10)$, of the remaining zigzag curvatures can be 
directly computed from the experimentally measured surface strains. However, a highly accurate estimate of $\mathrm{M}_{\alpha i}(\alpha=9,10)$ can be made through a preliminary iFEM analysis (i.e., the stage III. iFEM in Figure 3) using the continuous in situ strain measures ( $\mathbf{E}, \mathbf{K}, \mathbf{M}^{j}$ ) and omitting the contributions of the transverse-shear strain measures, G. Although this preliminary iFEM analysis may not provide an accurate enough solution for the deflection, it can provide promising solutions for bending and zigzag rotations even for thick laminates. Therefore, the main aim is to compute only $\mathrm{M}_{\alpha i}(\alpha=9,10)$ by taking relevant derivatives of zigzag rotations predicted through the preliminary iFEM analysis.

During the stage IV. SEA (refer to Figure 3), the discrete quantities $\mathrm{M}_{\alpha i}(\alpha=9,10)$ can be mapped on the smooth functions and the $\mathrm{C}^{1}$-continuous quantities; i.e., $\mathrm{M}_{\alpha}(\alpha=9,10)$, can be obtained by performing an additional SEA. Thus, the firstorder derivatives of $\mathbf{E}, \mathbf{K}$, and $\mathbf{M}$ can finally be computed and subsequently inserted in Eqs. (18a-c) to obtain the transverse-shear strain measures, G. As described in Figure 3, the overall outcome of this computational procedure will provide the continuous form of all experimental section strains, e.g., $\boldsymbol{\varepsilon}=\left[\begin{array}{llll}\mathbf{E} & \mathbf{K} & \mathbf{M}^{j} & \mathbf{G}\end{array}\right]$. Note that in order to obtain an accurate estimate of transverse-shear strain measures, the present iFEM methodology requires knowledge of all mechanical properties of the material lay-up, which is not the case for the iFEM-RZT formulation presented in [45].

\subsection{The weighted-least-squares functional}

Accounting for the membrane, bending, zigzag, and transverse-shear deformations of the RZT and adopting the iFEM methodology [26, 27, and 46] as general basis, a weighted-least-squares functional, $\Phi(\mathbf{u})$, can be defined as

$\Phi(\mathbf{u})=\sum_{\alpha=1}^{13} w_{\alpha} \varphi_{\alpha}$

where $w_{\alpha}(\alpha=1-13)$ are positive valued weighting coefficients associated with the individual section strains and the functional $\varphi_{\alpha}(\alpha=1-13)$ is the least-squares functional of in situ section strains ( $\boldsymbol{\varepsilon}$ or $\boldsymbol{\varepsilon}_{i}$ ) and kinematic variables $\mathbf{u}$. If the discrete 
experimental strain measures, $\boldsymbol{\varepsilon}_{i}$, are directly used in iFEM analysis, the least-squares functional becomes $\varphi_{\alpha}=\varphi_{\alpha}\left(\mathbf{u}, \boldsymbol{\varepsilon}_{i}\right)(\alpha=1-13)$ and can be defined by the normalized Euclidean norms given as

$$
\begin{aligned}
& \varphi_{\alpha} \equiv \frac{1}{n} \sum_{i=1}^{n}\left[e_{\alpha}(\mathbf{u})_{i}-\mathrm{E}_{\alpha i}\right]^{2} \quad(\alpha=1,2,3) \\
& \varphi_{\alpha} \equiv \frac{(2 h)^{2}}{n} \sum_{i=1}^{n}\left[\kappa_{\alpha}(\mathbf{u})_{i}-\mathrm{K}_{\alpha i}\right]^{2} \quad(\alpha=4,5,6) \\
& \varphi_{\alpha} \equiv \frac{1}{n} \sum_{i=1}^{n}\left[\mu_{\alpha}^{(k)}(\mathbf{u})_{i}-\mathrm{M}_{\alpha i}^{j}\right]^{2} \quad(\alpha=7,8,9) \\
& \varphi_{\alpha} \equiv \frac{1}{n} \sum_{i=1}^{n}\left[\gamma_{\alpha}(\mathbf{u})_{i}-\Gamma_{\alpha i}\right]^{2} \quad(\alpha=10,11) \\
& \varphi_{\alpha} \equiv \frac{1}{n} \sum_{i=1}^{n}\left[\eta_{\alpha}(\mathbf{u})_{i}-\mathrm{H}_{\alpha i}\right]^{2} \quad(\alpha=12,13)
\end{aligned}
$$

where $1<k<N$ and $j=k$ or $(k-1)$. Instead, if the computational tool (as described in previous section) is used to perform SEA such that the continuous experimental strain measures, $\boldsymbol{\varepsilon}$, become available for $\mathrm{iFEM}$ analysis, the least-squares functional becomes $\varphi_{\alpha}=\varphi_{\alpha}(\mathbf{u}, \boldsymbol{\varepsilon})(\alpha=1-13)$ and can be expressed in terms of the dimensionless $\mathrm{L}_{2}$ squared norms given as

$$
\begin{aligned}
& \varphi_{\alpha} \equiv \frac{1}{A} \int_{A}\left[e_{\alpha}(\mathbf{u})-\mathrm{E}_{\alpha}\right]^{2} d A \quad(\alpha=1,2,3) \\
& \varphi_{\alpha} \equiv \frac{(2 h)^{2}}{A} \int_{A}\left[\kappa_{\alpha}(\mathbf{u})-\mathrm{K}_{\alpha}\right]^{2} d A \quad(\alpha=4,5,6) \\
& \varphi_{\alpha} \equiv \frac{1}{A} \int_{A}\left[\mu_{\alpha}^{(k)}(\mathbf{u})-\mathrm{M}_{\alpha}^{j}\right]^{2} d A \quad(\alpha=7,8,9) \\
& \varphi_{\alpha} \equiv \frac{1}{A} \int_{A}\left[\gamma_{\alpha}(\mathbf{u})-\Gamma_{\alpha}\right]^{2} d A \quad(\alpha=10,11) \\
& \varphi_{\alpha} \equiv \frac{1}{A} \int_{A}\left[\eta_{\alpha}(\mathbf{u})-\mathrm{H}_{\alpha}\right]^{2} d A \quad(\alpha=12,13)
\end{aligned}
$$


The proper usage of the weighting constants $w_{\alpha}(\alpha=1-13)$ in Eq. (20) is crucial for those applications involving few locations of strain sensors. The weighting constants are set as $w_{\alpha}=1(\alpha=1-13)$ for Eq. (21) or (22), if every analytic section strain has a comparable experimental value ( $\boldsymbol{\varepsilon}$ or $\boldsymbol{\varepsilon}_{i}$ ). In the case of lacking an experimental strain component, the corresponding weighting coefficient is adjusted to a small number, e.g., $\lambda=10^{-5}$, and Eq. (21) or (22) take on the reduced form defined by the $\mathrm{L}_{2}$ squared norms

$$
\begin{aligned}
& \varphi_{\alpha} \equiv \frac{1}{A} \int_{A}\left[e_{\alpha}(\mathbf{u})\right]^{2} d A \quad(\alpha=1,2,3) \\
& \varphi_{\alpha} \equiv \frac{(2 h)^{2}}{A} \int_{A}\left[\kappa_{\alpha}(\mathbf{u})\right]^{2} d A \quad(\alpha=4,5,6) \\
& \varphi_{\alpha} \equiv \frac{1}{A} \int_{A}\left[\mu_{\alpha}^{(k)}(\mathbf{u})\right]^{2} d A \quad(\alpha=7,8,9) \\
& \varphi_{\alpha} \equiv \frac{1}{A} \int_{A}\left[\gamma_{\alpha}(\mathbf{u})\right]^{2} d A \quad(\alpha=10,11) \\
& \varphi_{\alpha} \equiv \frac{1}{A} \int_{A}\left[\eta_{\alpha}(\mathbf{u})\right]^{2} d A \quad(\alpha=12,13)
\end{aligned}
$$

Furthermore, the iFEM methodology allows the use of "strain-less" inverse elements that have none of the experimental section strain measures. Every squared norm given in Eqs. (23a-e) has to be multiplied by the small weighting constants $w_{\alpha}=\lambda(\alpha=1-13)$ for a strain-less element. Hence, an iFEM model can have very sparse in situ strain-sensor data, and yet the compulsory interpolation connectivity can still be preserved between the inverse elements that have strain data.

It is important to note that the weighted-least-squares functional, $\Phi(\mathbf{u})$, given by Eq. (20) can be simply transformed to the original iFEM formulation [28] based on the FSDT by setting the weighting coefficients associated with the zigzag strain measures and second transverse-shear strain measures to zero. Moreover, a similar transformation of the present iFEM formulation can be made for the iFEM-RZT formulation proposed in [45] by simply setting the weighting coefficients 
corresponding to the first transverse-shear strain measures to zero in Eq. (20). These aspects of the $\Phi(\mathbf{u})$ functional demonstrate the generality of the present enhanced iFEM formulation.

\section{A Three-Node Triangular Inverse-Shell Element Formulation based on RZT}

A three-node triangular inverse-shell element, named "i3-RZT", is developed on the basis of an improved iFEM algorithm. The inverse-element formulation is derived using the Tessler-Dong interdependent interpolation concept $[50,51]$. The key concept originates from the RZT beam-frame formulation, from which constraint equations are devised and imposed to each edge of an unconstrained triangular element. The unconstrained element is a six-node triangular element with seven displacement degrees of freedom (DOF) on the corner nodes and three displacement DOF on the mid-side nodes. After the application of relevant constant shear edge constraint conditions, the displacement DOF on the mid-nodes are condensed out into the corner nodes and anisoparametric shape functions [52] become available for the i3-RZT element. Finally, the i3-RZT inverse-element has nine displacement DOF per node (only corner nodes) including drilling rotations $\left(\theta_{z i}\right)$ and artificial zigzag rotations $\left(\psi_{z i}\right)$, as shown in Figure 4.

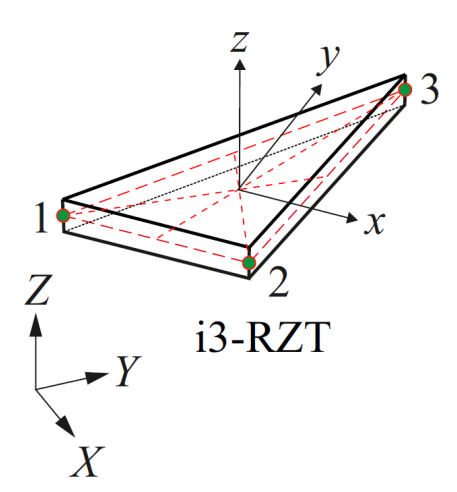

(a)

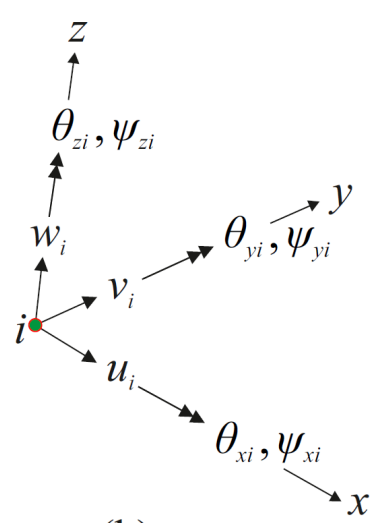

(b)

Figure 4. (a) Three-node triangular inverse-shell element, i3-RZT, shown within global and local coordinate systems; (b) Nodal DOF in local coordinate system. 
Due to the presence of drilling rotations, the i3-RZT element has two beneficial aspects: (1) Singular solutions can be basically avoided when modelling complicated shell structures; and (2) the i3-RZT element has fewer propensity against shear locking for membrane problems. Furthermore, it is much easier to implement the i3-RZT element than the unconstrained element because each single node has the same number of displacement DOF. An orthogonal coordinate system $(x, y, z)$ with its origin $(0,0,0)$ located at the centroid of the mid-plane triangle is defined as an element (local) coordinate system. In the following formulation, these local coordinates $(x, y, z)$ are related with the laminate (plate) coordinates $\left(x_{1}, x_{2}, z\right)$ used to define the kinematic relations of RZT in Section 2. Thus, the coordinates $(x, y) \equiv\left(x_{1}, x_{2}\right)$ are the in-plane coordinates and $z \in[-h,+h]$ defines the thickness coordinate. It is necessary to assemble element equations into a global system of equations. For this purpose, appropriate transformation matrices $\mathbf{T}^{e}$ that defines the local-to-global transformations can be readily constructed by utilizing the global coordinates $(X, Y, Z)$ of the element nodes. The basic steps to assign the local coordinate system at the centroid of mid-plane triangular and construct the transformation matrix $\mathbf{T}^{e}$ are summarized in Appendix A.

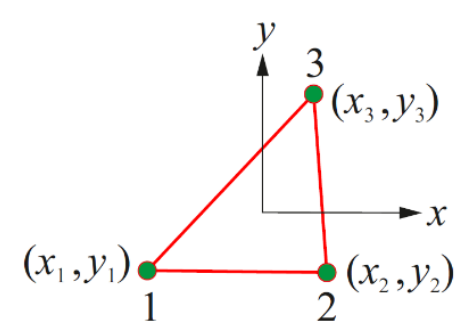

(a)

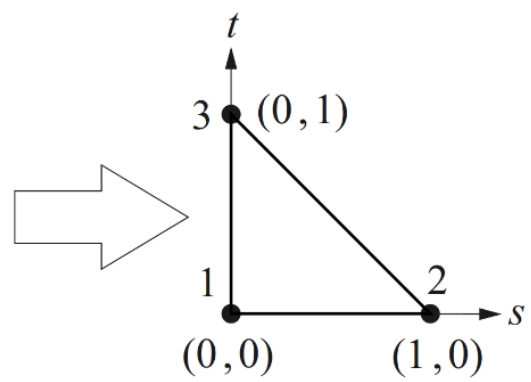

(b)

Figure 5. (a) Mid-plane $(x, y)$-reference surface and nodal coordinates of i3-RZT element; (b) Parent element in isoparametric coordinates.

The $(x, y)$ reference plane of the i3-RZT element can be uniquely defined in terms of bilinear mapping functions as

$$
\begin{aligned}
& x(s, t)=(1-s-t) x_{1}+s x_{2}+t x_{3} \\
& y(s, t)=(1-s-t) y_{1}+s y_{2}+t y_{3}
\end{aligned}
$$


where $s$ and $t$ are dimensionless isoparametric coordinates and $\left(x_{i}, y_{i}\right)(i=1-3)$ are the local nodal coordinates of the element, as illustrated in Figure 5. This definition is necessary for numerical Gauss integration of any functional on the surface of the element, $A^{e}$.

The nodal DOF, consisting of positive $x$ translations $u_{i}$, positive $y$ translations $v_{i}$, and positive counter clockwise drilling rotations $\theta_{z i}$, define the $u$ and $v$ mid-plane membrane displacements by

$$
\begin{aligned}
& u=\sum_{i=1}^{3}\left(N_{i} u_{i}+L_{i} \theta_{z i}\right) \\
& v=\sum_{i=1}^{3}\left(N_{i} v_{i}+M_{i} \theta_{z i}\right)
\end{aligned}
$$

where $N_{i}$ is the linear area-parametric coordinates of the triangle and the interpolation functions $L_{i}$ and $M_{i}$ are the anisoparametric shape functions that define the interaction between the hierarchical drilling rotation DOF and the membrane displacements of the element.

Besides, the transverse deflection $w$, two bending rotations $\theta_{\alpha}(\alpha=1,2)$, and two zigzag rotations $\psi_{\alpha}(\alpha=1,2)$ are defined by the nodal DOF of positive $z$ translation $w_{i}$ and positive counter clockwise rotations around the $x$ - and $y$-axes, $\theta_{x i}, \psi_{x i}$ and $\theta_{y i}, \psi_{y i}$. These kinematic variables are interpolated as

$$
\begin{aligned}
& w=\sum_{i=1}^{3}\left[N_{i} w_{i}-L_{i}\left(\theta_{x i}-\psi_{x i}\right)-M_{i}\left(\theta_{y i}-\psi_{y i}\right)\right] \\
& \theta_{1}=\sum_{i=1}^{3} N_{i} \theta_{y i} \\
& \theta_{2}=-\sum_{i=1}^{3} N_{i} \theta_{x i} \\
& \psi_{1}=\sum_{i=1}^{3} N_{i} \psi_{y i}
\end{aligned}
$$


$\psi_{2}=-\sum_{i=1}^{3} N_{i} \psi_{x i}$

where area-parametric coordinates, $N_{i}$, interpolate bending and zigzag rotations and the anisoparametric shape functions, $L_{i}$ and $M_{i}$, combine $z$ translation, bending, and zigzag rotation DOF altogether in the interpolation of the transverse deflection, $w$. In fact, these shape functions were originally developed by Tessler and Hughes [52] for a three-node plate element, MIN3 (Mindlin-type, three-nodes), and were used by many authors e.g., Versino et al. [53], Cerracchio et al. [44, 45]. The explicit forms can be defined as

$$
\left.\begin{array}{l}
N_{i}=\frac{b_{i} x+a_{i} y+c_{i}}{2 A_{e}} \\
M_{i}=\frac{N_{i}}{2}\left(a_{k} N_{j}-a_{j} N_{k}\right) \\
L_{i}=\frac{N_{i}}{2}\left(b_{k} N_{j}-b_{j} N_{k}\right)
\end{array}\right\}(i=1,2,3 ; j=2,3,1 ; k=3,1,2)
$$

with

$$
\left.\begin{array}{l}
a_{i}=x_{k}-x_{j} \\
b_{i}=y_{j}-y_{k} \\
c_{i}=x_{j} y_{k}-x_{k} y_{j}
\end{array}\right\}(i=1,2,3 ; j=2,3,1 ; k=3,1,2)
$$

Taking the relevant partial derivatives of Eqs. (25-26), then substituting these derivatives into Eqs. (7a-c) and (9a-b), gives rise to explicit definitions of membrane strain measures, bending curvatures, zigzag strain measures, and first and second transverse-shear strain measures in terms of the element nodal displacement vector, $\mathbf{u}^{e}$, as

$$
\begin{aligned}
& \mathbf{e}\left(\mathbf{u}^{e}\right)=\left[\begin{array}{lll}
\mathbf{B}_{1} \mathbf{u}^{e} & \mathbf{B}_{2} \mathbf{u}^{e} & \mathbf{B}_{3} \mathbf{u}^{e}
\end{array}\right]^{T} \\
& \boldsymbol{\kappa}\left(\mathbf{u}^{e}\right)=\left[\begin{array}{lll}
\mathbf{B}_{4} \mathbf{u}^{e} & \mathbf{B}_{5} \mathbf{u}^{e} & \mathbf{B}_{6} \mathbf{u}^{e}
\end{array}\right]^{T} \\
& \boldsymbol{\mu}^{(k)}\left(\mathbf{u}^{e}\right)=\left[\begin{array}{lll}
\phi_{1}^{(k)} \mathbf{B}_{7} \mathbf{u}^{e} & \phi_{2}^{(k)} \mathbf{B}_{8} \mathbf{u}^{e} & \mathbf{H}_{\phi}^{(k)} \mathbf{B}_{9} \mathbf{u}^{e}
\end{array}\right]^{T} \\
& \boldsymbol{\gamma}\left(\mathbf{u}^{e}\right)=\left[\begin{array}{lll}
\mathbf{B}_{10} \mathbf{u}^{e} & \mathbf{B}_{11} \mathbf{u}^{e}
\end{array}\right]^{T}
\end{aligned}
$$


$\boldsymbol{\eta}\left(\mathbf{u}^{e}\right)=\left[\begin{array}{ll}\mathbf{B}_{12} \mathbf{u}^{e} & \mathbf{B}_{13} \mathbf{u}^{e}\end{array}\right]^{T}$

with

$\mathbf{H}_{\phi}^{(k)}=\left[\phi_{1}^{(k)} \quad \phi_{2}^{(k)}\right]$

$\mathbf{u}^{e}=\left[\begin{array}{lll}\mathbf{u}_{1}^{e} & \mathbf{u}_{2}^{e} & \mathbf{u}_{3}^{e}\end{array}\right]^{T}$

$\mathbf{u}_{i}^{e}=\left[\begin{array}{lllllllll}u_{i} & v_{i} & w_{i} & \theta_{x i} & \theta_{y i} & \theta_{z i} & \psi_{x i} & \psi_{y i} & \psi_{z i}\end{array}\right]^{T} \quad(i=1-3)$

and where the matrices $\mathbf{B}_{\alpha}(\alpha=1-13)$ contain derivatives of the shape functions (refer to Appendix B).

Firstly, the continuous section strains $\boldsymbol{\varepsilon}$ can be obtained using the computational tool described in Section 2.3. Secondly, the analytic section strains can be calculated as given in Eqs. (28a-e). Thirdly, substituting these experimental and analytical section strains into the weighted-least-squares functional, given by Eqs. (20) and (22a-e), give rise to

$\Phi_{e}\left(\mathbf{u}^{e}\right)=\sum_{\alpha=1}^{13} w_{\alpha} \varphi_{\alpha}\left(\mathbf{u}^{e}, \boldsymbol{\varepsilon}\right)$

where the functional $\Phi_{e}\left(\mathbf{u}^{e}\right)$ is defined for an individual i3-RZT element. Finally, minimizing this functional, $\Phi_{e}\left(\mathbf{u}^{e}\right)$, with respect to the nodal displacement DOF, $\mathbf{u}^{e}$, reveals that

$\frac{\partial \Phi_{e}\left(\mathbf{u}^{e}\right)}{\partial \mathbf{u}^{e}}=\Gamma^{e} \mathbf{u}^{e}-\boldsymbol{\varepsilon}^{e}=0 \Rightarrow \Gamma^{e} \mathbf{u}^{e}=\boldsymbol{\varepsilon}^{e}$

where $\boldsymbol{\Gamma}^{e}$ is the element left-hand-side matrix; $\boldsymbol{\varepsilon}^{e}$ is the element right-hand-side vector, which is a function of the experimental strain values; and $\mathbf{u}^{e}$ is the nodal displacement vector of the element.

The $\Gamma^{e}$ matrix combines the contribution of every analytic section strain component and its corresponding weighting constant $w_{\alpha}(\alpha=1-13)$ and is given by

$\boldsymbol{\Gamma}^{e}=\sum_{\alpha=1}^{13} w_{\alpha} \mathbf{k}_{\alpha}^{e}$ 
where $\mathbf{k}_{\alpha}^{e}(\alpha=1-13)$ matrices denote the contribution of each analytic section strain component and can be explicitly written in terms of the $\mathbf{B}_{\alpha}(\alpha=1-13)$ matrices as

$$
\begin{aligned}
& \mathbf{k}_{\alpha}^{e}=\frac{1}{A_{e}} \int_{A_{e}} \mathbf{B}_{\alpha}^{T} \mathbf{B}_{\alpha} d x d y \quad(\alpha=1-3,10-13) \\
& \mathbf{k}_{\alpha}^{e}=\frac{(2 h)^{2}}{A_{e}} \int_{A_{e}} \mathbf{B}_{\alpha}^{T} \mathbf{B}_{\alpha} d x d y \quad(\alpha=4-6) \\
& \mathbf{k}_{7}^{e}=\frac{\left(\phi_{1}^{(k)}\right)^{2}}{A_{e}} \int_{A_{e}} \mathbf{B}_{7}^{T} \mathbf{B}_{7} d x d y \\
& \mathbf{k}_{8}^{e}=\frac{\left(\phi_{2}^{(k)}\right)^{2}}{A_{e}} \int_{A_{e}} \mathbf{B}_{8}^{T} \mathbf{B}_{8} d x d y \\
& \mathbf{k}_{9}^{e}=\frac{1}{A_{e}} \int_{A_{e}} \mathbf{B}_{9}^{T}\left(\mathbf{H}_{\phi}^{(k)}\right)^{T} \mathbf{H}_{\phi} \mathbf{B}_{9} d x d y
\end{aligned}
$$

Once the left hand side matrix $\boldsymbol{\Gamma}^{e}$ is constructed using the Eqs. (31a-f), an artificial contribution matrix $\boldsymbol{\Gamma}_{\psi z}$ of the artificial zigzag rotations $\psi_{z i}(i=1-3)$ must be added to the $\Gamma^{e}$ matrix to avoid singular solutions. The procedure of constructing the matrix $\Gamma_{\psi z}$ is described in Appendix C.

The $\varepsilon^{e}$ vector couple the contribution of every experimental section strain component and its corresponding weighting constant $w_{\alpha}(\alpha=1-13)$ and is given by

$$
\boldsymbol{\varepsilon}^{e}=\sum_{\alpha=1}^{13} w_{\alpha} \mathbf{f}_{\alpha}^{e}
$$

where $\mathbf{f}_{\alpha}^{e}(\alpha=1-13)$ vectors denote the contribution of each experimental section strain component and can be explicitly written in terms of the measured section-strain values as

$$
\begin{aligned}
& \mathbf{f}_{\alpha}^{e}=\frac{1}{A_{e}} \int_{A_{e}} \mathbf{B}_{\alpha}^{T} \mathrm{E}_{\alpha} d x d y \quad(\alpha=1-3) \\
& \mathbf{f}_{\alpha}^{e}=\frac{(2 h)^{2}}{A_{e}} \int_{A_{e}} \mathbf{B}_{\alpha}^{T} \mathrm{~K}_{\alpha} d x d y \quad(\alpha=4-6)
\end{aligned}
$$




$$
\begin{aligned}
\mathbf{f}_{7}^{e} & =\frac{\phi_{1}^{(k)}}{A_{e}} \int_{A_{e}} \mathbf{B}_{7}^{T} \mathbf{M}_{7}^{j} d x d y \\
\mathbf{f}_{8}^{e} & =\frac{\phi_{2}^{(k)}}{A_{e}} \int_{A_{e}} \mathbf{B}_{8}^{T} \mathbf{M}_{8}^{j} d x d y \\
\mathbf{f}_{9}^{e} & =\frac{1}{A_{e}} \int_{A_{e}} \mathbf{B}_{9}^{T}\left(\mathbf{H}_{\phi}^{(k)}\right)^{T} \mathbf{M}_{9}^{j} d x d y \\
\mathbf{f}_{\alpha}^{e} & =\frac{1}{A_{e}} \int_{A_{e}} \mathbf{B}_{\alpha}^{T} \Gamma_{\alpha} d x d y \quad(\alpha=10,11) \\
\mathbf{f}_{\alpha}^{e} & =\frac{1}{A_{e}} \int_{A_{e}} \mathbf{B}_{\alpha}^{T} \mathrm{H}_{\alpha} d x d y \quad(\alpha=12,13)
\end{aligned}
$$

After the local matrix equations are defined for individual inverse elements, they can be assembled into the global matrix equation of the discretized structure as

$$
\mathbf{A} \mathbf{U}=\mathbf{Q}
$$

with

$$
\begin{aligned}
& \mathbf{A}=\Omega_{e=1}^{n e l}\left[\left(\mathbf{T}^{e}\right)^{T} \boldsymbol{\Gamma}^{e} \mathbf{T}^{e}\right] \\
& \mathbf{U}=\Omega_{e=1}^{n e l}\left[\left(\mathbf{T}^{e}\right)^{T} \mathbf{u}^{e}\right] \\
& \mathbf{Q}=\Omega_{e=1}^{n e l}\left[\left(\mathbf{T}^{e}\right)^{T} \boldsymbol{\varepsilon}^{e}\right]
\end{aligned}
$$

where the global left-hand-side matrix $\mathbf{A}$ is symmetric and independent of the in situ strain data, the global displacement vector $\mathbf{U}$ contains the global displacement DOF of all the nodes, and the global right-hand-side vector $\mathbf{Q}$ is a function of the experimental strain measurements. In Eqs. (33b-d), the parameter nel denotes the total number of the inverse elements and the symbol $\Omega$ represents assembly of the element matrices/vectors into global matrices/vectors.

In fact, the matrix $\mathbf{A}$ is singular (non-invertible) because it contains the rigid body mode of the discretized structure. Once the problem-specific constraint boundary conditions are prescribed, the final matrix equations can be reduced from Eq. (33a) as 
$\mathbf{A}_{R} \mathbf{U}_{R}=\mathbf{Q}_{R}$

where $\mathbf{A}_{R}$ is a positive definite matrix, i.e., always non-singular (invertible). The Eq. (34) can be solved very quickly because the matrix $\mathbf{A}_{R}$ doesn't change for a specified distribution of strain sensors, and its inverse has to be computed only once during the shape-sensing analysis. However, the vector $\mathbf{Q}_{R}$ is dependent on the experimentally measured surface strains, therefore it must be updated at each strain-data acquisition increment. Finally, the matrix-vector multiplication $\mathbf{A}_{R}^{-1} \mathbf{Q}_{R}$ gives rise to the unknown displacement DOF vector $\mathbf{U}_{R}$, which provides the total deformation of the structure at any real time. One can obtain the full-field strains and displacements throughout the structure by using the calculated displacement values. Furthermore, the constitutive relationship between stress and strain (i.e., the generalized Hooke's law for the $k$-th orthotropic lamina) can allow prediction of stress distribution everywhere within the laminate. Finally, these stress distributions can be utilized with a suitable failure criterion for composite materials, allowing the identification of the damage and/or failure locations for the SHM process.

\section{Numerical Examples}

A simply supported rectangular plate (laminate) with three different lamina stacking sequences (uniaxial, cross-ply and angle-ply) has been originally analyzed in [43] based on the analytical solution of the RZT plate theory. The authors obtained superior displacement and stress results in comparison to other solutions such as 3D elasticity theory [54], FSDT [40, 41], and the theory of Di Sciuva [55]. In Section 4.1, this problem is revisited to validate the accuracy of the enhanced iFEM formulation. After the validation case, the applicability of the present iFEM formulation to more complex composite/sandwich structures is demonstrated by analyzing a wedge structure with a hole near one of the clamped ends (refer to Section 4.2). The detailed distributions of the displacements and stresses are examined for both example problems.

\subsection{Simply supported rectangular laminates}

As depicted in Figure 6, the plate has a length of $\mathrm{a}=1 \mathrm{~m}$, height of $\mathrm{b}=1 \mathrm{~m}$, and uniform thickness of $2 \mathrm{~h}=0.2 \mathrm{~m}$. The plate is subjected to a sinusoidal varying 
transverse pressure, $q(\mathrm{X}, \mathrm{Y})=q_{0} \sin (\pi \mathrm{X} / \mathrm{a}) \sin (\pi \mathrm{Y} / \mathrm{b})$, where the pressure magnitude is $q_{0}=-1 \mathrm{MPa}$. As presented in Figure 6, the kinematic variables are defined as follows: $\mathrm{U}, \mathrm{V}$, and $\mathrm{W}$ represent the translations along the coordinate directions $\mathrm{X}, \mathrm{Y}$, and $\mathrm{Z}$, respectively; $\theta_{\mathrm{X}}, \theta_{\mathrm{Y}}$ and $\psi_{\mathrm{X}}, \psi_{\mathrm{Y}}$ represent bending and zigzag rotations around the positive $\mathrm{X}$ and $\mathrm{Y}$ directions, respectively.

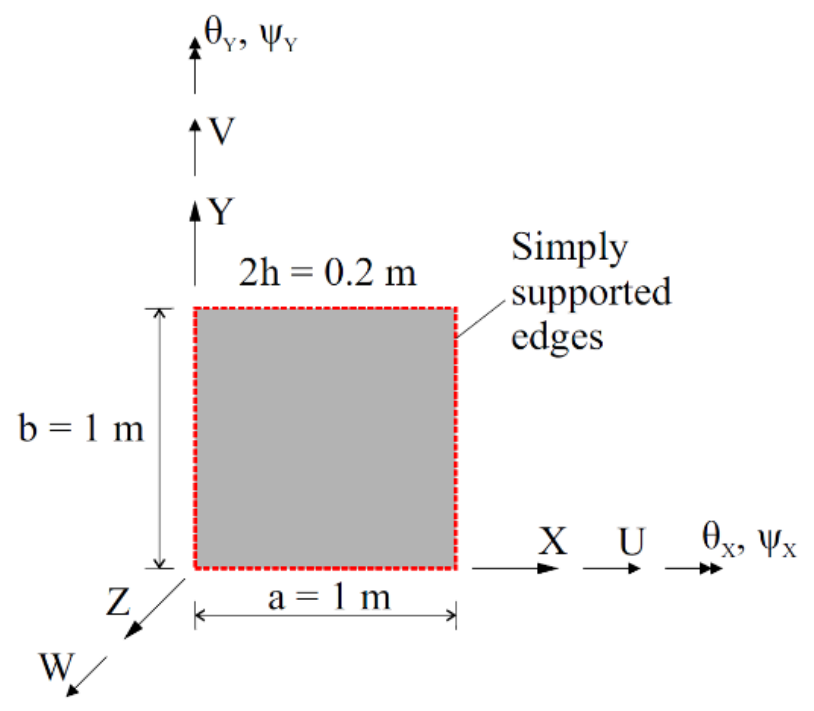

Figure 6. Simply supported plate subjected to sinusoidal varying pressure.

The four edges of the plate are simply supported and the following kinematic boundary conditions satisfy the simply supported boundary condition of the plate: For cross-ply and uniaxial laminates, the kinematic boundary conditions along $\mathrm{X}=0$ and $\mathrm{X}=\mathrm{a}$ are $\mathrm{V}=\mathrm{W}=\theta_{\mathrm{X}}=\psi_{\mathrm{X}}=0$

and along $\mathrm{Y}=0$ and $\mathrm{Y}=\mathrm{b}$ are

$\mathrm{U}=\mathrm{W}=\theta_{\mathrm{Y}}=\psi_{\mathrm{Y}}=0$

For anti-symmetric angle-ply laminates, the kinematic boundary conditions along $\mathrm{X}=0$ and $\mathrm{X}=\mathrm{a}$ are

$\mathrm{U}=\mathrm{W}=\theta_{\mathrm{X}}=\psi_{\mathrm{X}}=0$

and along $\mathrm{Y}=0$ and $\mathrm{Y}=\mathrm{b}$ are

$\mathrm{V}=\mathrm{W}=\theta_{\mathrm{Y}}=\psi_{\mathrm{Y}}=0$ 
Three different laminates (I, II, III) are considered for representing relatively thick laminated composite and sandwich plates with a span-to-thickness ratio of $\mathrm{a} / 2 \mathrm{~h}=\mathrm{b} / 2 \mathrm{~h}=5$. Laminate $\mathrm{I}$ is a two-layer, cross-ply carbon-epoxy laminate. Laminate II is a three-layer sandwich laminate with uniaxial carbon-epoxy face sheets and a thick, closed cell polyvinyl chloride (PVC) core, where PVC is represented as an isotropic material. Laminate III is a five-layer, angle-ply sandwich laminate with carbon-epoxy face sheets and a thick PVC core. The mechanical material properties and the stacking sequences of the laminates are listed in Tables 1-2, respectively.

Table 1. Mechanical properties of orthotropic and isotropic materials.

\begin{tabular}{llll}
\hline Lamina material & $\begin{array}{l}\text { Young's } \\
\text { modulus [GPa] }\end{array}$ & Poisson's ratio & $\begin{array}{l}\text { Shear modulus } \\
{[\mathrm{GPa}]}\end{array}$ \\
\hline \multirow{4}{*}{$\begin{array}{l}\text { Carbon-epoxy } \\
\text { C unidirectional }\end{array}$} & $E_{1}^{(k)}=157.9$ & $v_{12}^{(k)}=0.32$ & $G_{12}^{(k)}=5.930$ \\
& $E_{2}^{(k)}=9.584$ & $v_{13}^{(k)}=0.32$ & $G_{13}^{(k)}=5.930$ \\
composite & $E_{3}^{(k)}=9.584$ & $v_{23}^{(k)}=0.49$ & $G_{23}^{(k)}=3.227$ \\
$\mathrm{P} \quad$ PVC core & $E^{(k)}=0.104$ & $v^{(k)}=0.3$ & $G^{(k)}=0.04$ \\
\hline
\end{tabular}

Table 2. Laminate stacking sequences in the positive $\mathrm{Z}$ direction.

\begin{tabular}{lllll}
\hline \multicolumn{2}{l}{ Laminate } & $\begin{array}{l}\text { Normalized lamina } \\
\text { thickness, } \mathrm{h}^{(k)} / \mathrm{h}\end{array}$ & $\begin{array}{l}\text { Lamina } \\
\text { materials }\end{array}$ & $\begin{array}{l}\text { Lamina } \\
\text { orientation }{ }^{\circ}{ }^{\circ}\end{array}$ \\
\hline I $\quad$ Cross-ply composite & $(0.5 / 0.5)$ & $(\mathrm{C} / \mathrm{C})$ & $(0 / 90)$ \\
II $\quad$ Uniaxial sandwich & $(0.1 / 0.8 / 0.1)$ & $(\mathrm{C} / \mathrm{P} / \mathrm{C})$ & $(0 / 0 / 0)$ \\
& & $(0.05 / 0.05 / 0.8 / 0.05 /$ & $(\mathrm{C} / \mathrm{C} / \mathrm{P} / \mathrm{C} / \mathrm{C})$ & $(30 /-45 / 0 / 45 /-$ \\
III Angle-ply sandwich & $0.05)$ & & $30)$ \\
\hline
\end{tabular}

To establish an accurate reference solution, a convergence study was performed using direct RZT-based FEM analyses utilizing an in-house FEM code. The most refined mesh consisted of 10000 uniformly distributed triangular elements that possessed 35707 DOF. For each laminate (I, II, III), comparisons of the normalized central deflection are listed in Table 3, where the normalization factor of $10^{2} D_{11} / q_{0} \mathrm{a}^{4}$ is used with $D_{11}$ denoting the bending stiffness coefficient. These results demonstrate that the 
high-fidelity RZT-based FEM analyses predict plate displacements that are comparably accurate to those of the RZT analytical solutions [43]. Therefore, the highfidelity FEM deflections and rotations are used to compute the simulated strain-sensor strains.

Table 3. Normalized central deflection, $\overline{\mathrm{w}}=\left(10^{2} D_{11} / q_{0} \mathrm{a}^{4}\right) \mathrm{W}(0.5 \mathrm{a}, 0.5 \mathrm{~b})$.

\begin{tabular}{llll}
\hline Laminate & $\begin{array}{l}\text { Normalization Factor } \\
\left(10^{2} D_{11} / q_{0} \mathrm{a}^{4}\right)\end{array}$ & $\begin{array}{l}\text { RZT Analytic }(\overline{\mathrm{w}}) \\
{[43]}\end{array}$ & FEM $(\overline{\mathrm{w}})$ \\
\hline I & 5617.72 & 1.219 & 1.219 \\
II & 5173.04 & 29.785 & 29.775 \\
III & 2448.38 & 14.105 & 14.101 \\
\hline
\end{tabular}

Table 4. Maximum deflections and von Mises stresses of the plate.

\begin{tabular}{lll}
\hline Laminate & $\mathrm{W}_{\max }^{\mathrm{FEM}}[\mathrm{m}]$ & $\sigma_{\mathrm{v}, \max }^{\mathrm{FEM}}[\mathrm{Pa}]$ \\
\hline I & $-2.169 \times 10^{-4}$ & $1.306 \times 10^{7}$ \\
II & $-5.756 \times 10^{-3}$ & $1.397 \times 10^{6}$ \\
III & $-5.759 \times 10^{-3}$ & $2.587 \times 10^{7}$ \\
\hline
\end{tabular}

Table 5. Maximum bending and zigzag rotations of the plate

\begin{tabular}{lllll}
\hline Laminate & $\theta_{\mathrm{X}, \max }^{\mathrm{FEM}}[\mathrm{rad}]$ & $\theta_{\mathrm{Y}, \max }^{\mathrm{FEM}}[\mathrm{rad}]$ & $\psi_{\mathrm{X}, \max }^{\mathrm{FEM}}[\mathrm{rad}]$ & $\psi_{\mathrm{Y}, \max }^{\mathrm{FEM}}[\mathrm{rad}]$ \\
\hline I & $5.128 \times 10^{-4}$ & $5.128 \times 10^{-4}$ & $5.520 \times 10^{-5}$ & $5.520 \times 10^{-5}$ \\
II & $4.009 \times 10^{-3}$ & $1.913 \times 10^{-3}$ & $1.415 \times 10^{-2}$ & $1.607 \times 10^{-2}$ \\
III & $2.732 \times 10^{-3}$ & $1.571 \times 10^{-3}$ & $1.538 \times 10^{-2}$ & $1.652 \times 10^{-2}$ \\
\hline
\end{tabular}

Moreover, for each laminate (I, II, III), maximum deflection $\mathrm{W}_{\max }^{\mathrm{FEM}}$, von Mises stress $\sigma_{\mathrm{v}, \max }^{\mathrm{FEM}}$, bending rotations $\theta_{\mathrm{X}, \max }^{\mathrm{FEM}}$ and $\theta_{\mathrm{Y}, \max }^{\mathrm{FEM}}$, and zigzag rotations $\psi_{\mathrm{X}, \max }^{\mathrm{FEM}}$ and $\psi_{\mathrm{Y}, \max }^{\mathrm{FEM}}$ obtained from the high-fidelity FEM solutions are listed in Tables 4-5, respectively. To assess the accuracy of the displacement, rotation, and stress responses, it would be convenient to use these reference values as normalization factors. 
In the present iFEM analysis, the strain rosettes are regularly distributed, and each strain rosette configuration pertains to a discretization with the same number of element subdivisions along the plate edges, $\mathrm{n}^{\mathrm{e}}$. Through the thickness coordinate, three strain rosettes are located at the centroid of each element; one on the top surface, one on the bottom surface, and one on the nearest interface to the bottom surface of the laminate. Figure 7 demonstrates an example of iFEM discretization, $n^{\mathrm{e}}=4$, for which the total number of strain rosettes is $64 \times 3$.

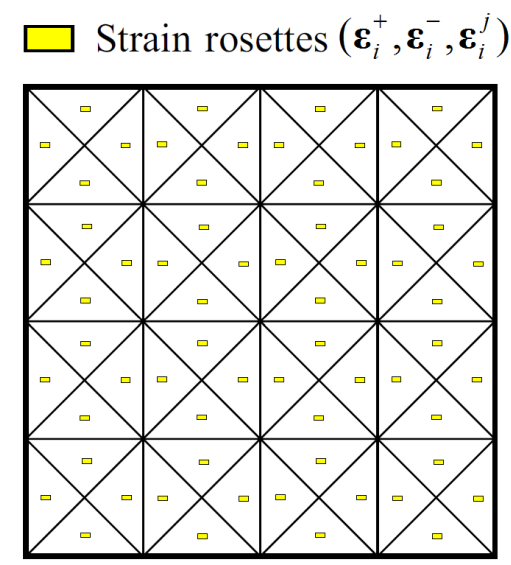

Figure 7. Strain rosette configuration of the simply supported plate for discretization

$$
\mathrm{n}^{\mathrm{e}}=4
$$

Utilizing the computational tool described in Section 2.3, the continuous in situ strain measures $\left(\mathbf{E}, \mathbf{K}, \mathbf{M}^{j}\right.$, and $\mathbf{G}$ ) are calculated for each laminate. Therefore, the continuous form of section strains $\boldsymbol{\varepsilon}$ is used in the following RZT-based iFEM analyses. The weighting constants associated with the membrane, bending, and zigzag strain measures are adjusted as $w_{\alpha}=1(\alpha=1-9)$ for all laminates. Moreover, the weighting constants corresponding to the transverse-shear strain measures are set to small values, $\lambda_{1}=10^{-4}$ and $\lambda_{2}=10^{-8}$. Note that the deformation of uniaxial laminate (I) produces larger first transverse-shear strains, $\gamma(\mathbf{u})$, than the second transverseshear strains, $\boldsymbol{\eta}(\mathbf{u})$. On the other hand, an opposite behavior is observed for the crossand angle-ply laminates (II and III). Therefore, the weighting coefficients associated with first and second transverse-shear strain measures are defined as $w_{10}=w_{11}=\lambda_{1}$ and $w_{12}=w_{13}=\lambda_{2}$ for laminate I, whereas they are set as $w_{10}=w_{11}=\lambda_{2}$ and $w_{12}=w_{13}=\lambda_{1}$ for laminates II and III. Although these weighting coefficients are small, the in situ 
transverse-shear strains play a critical role for deflection predictions of thick laminates. By including these in situ strain data in the $\Phi(\mathbf{u})$ functional, the deflections can be accurately reconstructed based on the match between analytical transverse-shear strain measures and their experimental values, even if their weighting constants are small. This is because the only strain measure that involves the quantities related to the deflection is transverse-shear strain. Small-valued weighting coefficients also enable the match between analytical curvatures (bending and zigzag) and their experimental counterparts to behave as a main contributor to the reconstruction of rotation variables. Hence, a higher accuracy level for bending and zigzag rotations can be maintained.

For each laminate (I, II, III), the deflections, bending and zigzag rotations, and von Mises stresses obtained from both iFEM and FEM analyses are normalized by absolute values of the FEM solutions listed in Tables 4-5. These normalized expressions are given as follows

$\bar{\chi}=\chi /\left|\chi_{\max }^{\mathrm{FEM}}\right|\left(\chi=\mathrm{W}, \theta_{\mathrm{X}}, \theta_{\mathrm{Y}}, \psi_{\mathrm{X}}, \psi_{\mathrm{Y}}, \sigma_{\mathrm{v}}\right)$

In Tables 6-8, the percent difference between iFEM and FEM predictions for the maximum values of the normalized rotations given in Eq. (37) are listed versus the number of element subdivisions $\mathrm{n}^{\mathrm{e}}$ for each laminate (I, II, III), respectively.

Table 6. Percent difference between the iFEM and FEM predictions for maximum values of the rotations of laminate $\mathrm{I}$.

\begin{tabular}{lcccc}
\hline \multirow{2}{*}{$\mathrm{n}^{\mathrm{e}}$} & \multicolumn{4}{c}{ Percent difference } \\
\cline { 2 - 5 } & $\bar{\theta}_{\mathrm{X}}$ & $\bar{\theta}_{\mathrm{Y}}$ & $\bar{\psi}_{\mathrm{X}}$ & $\bar{\Psi}_{\mathrm{Y}}$ \\
\hline 2 & 2.08 & 2.08 & 2.10 & 2.10 \\
4 & 0.70 & 0.70 & 0.93 & 0.93 \\
6 & 0.16 & 0.16 & 0.66 & 0.66 \\
8 & 0.05 & 0.05 & 0.26 & 0.26 \\
10 & 0.04 & 0.04 & 0.28 & 0.28 \\
\hline
\end{tabular}

These results show that the $\mathrm{iFEM} / \mathrm{i3}-\mathrm{RZT}$ predictions for all rotations convergence to the reference solution very quickly, even if only a few strain sensors are used. Note that the same accuracy level can be obtained for $\bar{\theta}_{\mathrm{X}}$ and $\bar{\theta}_{\mathrm{Y}}$ rotations, if the iFEM formulations proposed in [28] or [45] are used in the analyses. Although iMIN3 [28] 
cannot be used to attain the zigzag rotations, the iRZT3 [45] element produces same $\bar{\psi}_{\mathrm{X}}$ and $\bar{\psi}_{\mathrm{Y}}$ rotations as those obtained using i3-RZT element.

Table 7. Percent difference between the iFEM and FEM predictions for maximum values of the rotations of laminate II.

\begin{tabular}{lcccc}
\hline \multirow{2}{*}{$\mathrm{n}^{\mathrm{e}}$} & \multicolumn{4}{c}{ Percent difference } \\
\cline { 2 - 5 } & $\bar{\theta}_{\mathrm{X}}$ & $\bar{\theta}_{\mathrm{Y}}$ & $\bar{\psi}_{\mathrm{X}}$ & $\bar{\psi}_{\mathrm{Y}}$ \\
\hline 2 & 2.26 & 1.67 & 1.92 & 2.13 \\
4 & 0.78 & 0.53 & 0.66 & 0.72 \\
6 & 0.12 & 0.21 & 0.08 & 0.17 \\
8 & 0.09 & 0.02 & 0.06 & 0.06 \\
10 & 0.05 & 0.03 & 0.06 & 0.04 \\
\hline
\end{tabular}

Table 8. Percent difference between the iFEM and FEM predictions for maximum values of the rotations of laminate III.

\begin{tabular}{lllll}
\hline \multirow{2}{*}{$\mathrm{n}^{\mathrm{e}}$} & \multicolumn{4}{c}{ Percent difference } \\
\cline { 2 - 5 } & $\bar{\theta}_{\mathrm{X}}$ & $\bar{\theta}_{\mathrm{Y}}$ & $\bar{\psi}_{\mathrm{X}}$ & $\bar{\psi}_{\mathrm{Y}}$ \\
\hline 2 & 2.00 & 1.52 & 1.74 & 1.90 \\
4 & 0.75 & 0.55 & 0.66 & 0.70 \\
6 & 0.06 & 0.07 & 0.01 & 0.12 \\
8 & 0.11 & 0.05 & 0.09 & 0.08 \\
10 & 0.06 & 0.06 & 0.08 & 0.05 \\
\hline
\end{tabular}

To understand the merits of the enhanced iFEM methodology for composite and sandwich structures, we compare the deflection $(\overline{\mathrm{W}})$ predictions of present i3-RZT element with those of iRZT3 [45] and iMIN3 [28] elements. According to Tables 911, i3-RZT predicts the most convergent results to the reference FEM solutions for all three laminates. Note that the iRZT3 models produce incorrect results for uniaxial laminate (I), whereas it estimates accurate deflections for sandwich laminates (II and III). On the other hand, although the iMIN3 element predicts erroneous deflections for laminates II and III, it provides more accurate results for laminate I as compared to the 
iRZT3. Thus, these results reveal the superior capabilities of the i3-RZT element in comparison to the iRZT3 and iMIN3 elements.

Table 9. Percent difference between iFEM (i3-RZT, iRZT3 [45], and iMIN3 [28]) and FEM predictions for the $\overline{\mathrm{W}}$ displacements of laminate $\mathrm{I}$.

\begin{tabular}{llll}
\hline \multirow{2}{*}{$\mathrm{n}^{\mathrm{e}}$} & \multicolumn{3}{c}{ Percent Difference } \\
\cline { 2 - 4 } & $\mathrm{i}-\mathrm{RZT}$ & $\mathrm{iRZT3}$ & $\mathrm{iMIN3}$ \\
\hline 2 & 1.75 & 31.51 & 23.72 \\
4 & 1.57 & 33.38 & 25.35 \\
6 & 0.28 & 32.92 & 24.85 \\
8 & 0.15 & 32.93 & 24.84 \\
10 & 0.00 & 32.89 & 24.80 \\
\hline
\end{tabular}

Table 10. Percent difference between iFEM (i3-RZT, iRZT3 [45], and iMIN3 [28]) and FEM predictions for the $\overline{\mathrm{W}}$ displacements of laminate II.

\begin{tabular}{llll}
\hline \multirow{2}{*}{$\mathrm{n}^{\mathrm{e}}$} & \multicolumn{3}{c}{ Percent Difference } \\
\cline { 2 - 4 } & $\mathrm{i}$-RZT & $\mathrm{iRZT3}$ & $\mathrm{iMIN3}$ \\
\hline 2 & 1.42 & 1.34 & 83.39 \\
4 & 0.74 & 0.85 & 83.75 \\
6 & 0.08 & 0.19 & 83.64 \\
8 & 0.06 & 0.18 & 83.64 \\
10 & 0.00 & 0.12 & 83.63 \\
\hline
\end{tabular}

Table 11. Percent difference between iFEM (i3-RZT, iRZT3 [45], and iMIN3 [28]) and FEM predictions for the $\overline{\mathrm{W}}$ displacements of laminate III.

\begin{tabular}{llll}
\hline \multirow{2}{*}{$\mathrm{n}^{\mathrm{e}}$} & \multicolumn{3}{c}{ Percent Difference } \\
\cline { 2 - 4 } & i3-RZT & iRZT3 & iMIN3 \\
\hline 2 & 1.25 & 1.25 & 87.97 \\
4 & 0.74 & 0.73 & 88.20 \\
6 & 0.09 & 0.09 & 88.13 \\
8 & 0.06 & 0.06 & 88.12 \\
10 & 0.01 & 0.00 & 88.12 \\
\hline
\end{tabular}


In addition, for each simply supported square laminate (I, II, III), through-thethickness distributions of the in-plane displacements, normal and transverse-shear stresses are plotted in Figures 8-17, respectively, where the following normalization was used

$$
\begin{aligned}
& \overline{\mathrm{U}}(0,0.5 \mathrm{~b}, \mathrm{Z})=\left(10^{3} D_{11} / q_{0} \mathrm{a}^{4}\right) \mathrm{U}(0,0.5 \mathrm{~b}, \mathrm{Z}) \\
& \bar{\sigma}_{\mathrm{XX}}(0.55 \mathrm{a}, 0.6 \mathrm{~b}, \mathrm{Z})=\left(4 \mathrm{~h}^{2} / q_{0} \mathrm{a}^{2}\right) \sigma_{\mathrm{XX}}(0.55 \mathrm{a}, 0.6 \mathrm{~b}, \mathrm{Z}) \\
& \bar{\tau}_{\mathrm{XZ}}(0.11 \mathrm{a}, 0.2 \mathrm{~b}, \mathrm{Z})=\left(20 \mathrm{~h} / q_{0} \mathrm{a}^{2}\right) \tau_{\mathrm{XZ}}(0.11 \mathrm{a}, 0.2 \mathrm{~b}, \mathrm{Z})
\end{aligned}
$$

In these figures, the legend "Reference" represents the normalized high-fidelity FEM solutions whereas the legends "i3-RZT", "iRZT3", and "iMIN3" represent the normalized iFEM solutions. Moreover, the legend "i3-RZT (E)" represents the $\bar{\tau}_{\mathrm{XZ}}$ stress results that are obtained by integrating Cauchy's 3D equilibrium equations; therefore, these $\bar{\tau}_{\mathrm{Xz}}$ stress distributions are continuous through the thickness of the laminates (refer to Figures 10, 13, 16, and 17). Furthermore, the through-the-thickness distributions demonstrated in Figures 8-17 are obtained utilizing the iFEM model, $\mathrm{n}^{\mathrm{e}}=4$ (see Figure 7).

For the uniaxial laminate (I), the $\bar{U}$ and $\bar{\sigma}_{\mathrm{XX}}$ distributions are accurately computed by all three inverse elements as depicted in Figures 8 and 9. However, there are major differences in $\bar{\tau}_{\mathrm{XZ}}$ distributions (Figure 10), where i3-RZT (E) is best correlated with the reference solution, i3-RZT produces relatively accurate piecewise constant (average) stresses, and iRZT3 and iMIN3 significantly underestimate the transverseshear stresses.

For sandwich laminates (II and III), although iMIN3 provides accurate in-plane displacements at the top and bottom surfaces of the plate, it can only predict an average $\overline{\mathrm{U}}$ distribution through the thickness as shown in Figures 11 and 14. In contrast, the i3-RZT and iRZT3 predictions for the $\bar{U}$ distributions match very well with their corresponding reference solution and they exhibit a zigzag distribution through the thickness (Figures 11 and 14). In addition, the $\bar{\sigma}_{\mathrm{XX}}$ distributions are estimated 
accurately using both i3-RZT and iRZT3 elements, however the iMIN3 element produces erroneous normal stresses at the face sheets as depicted in Figures 12 and 15. Furthermore, the evaluation of $\bar{\tau}_{\mathrm{xz}}$ stresses in Figures 13, 16, and 17 demonstrates that i3-RZT and iRZT3 elements capture the correct average transverse shear stresses (i.e., evaluated from the constitutive relations) both in the face sheets and core of the sandwich laminates (II and III). Besides, the equilibrium-based method, i3-RZT (E), yields the most accurate solution of the transverse-shear stress distributions that are virtually indistinguishable from their corresponding reference solutions (refer to Figure 17). By contrast, the iMIN3 produces inaccurate evaluation of $\bar{\tau}_{\mathrm{Xz}}$ stresses compared to the reference solution in Figures 13 and 16.

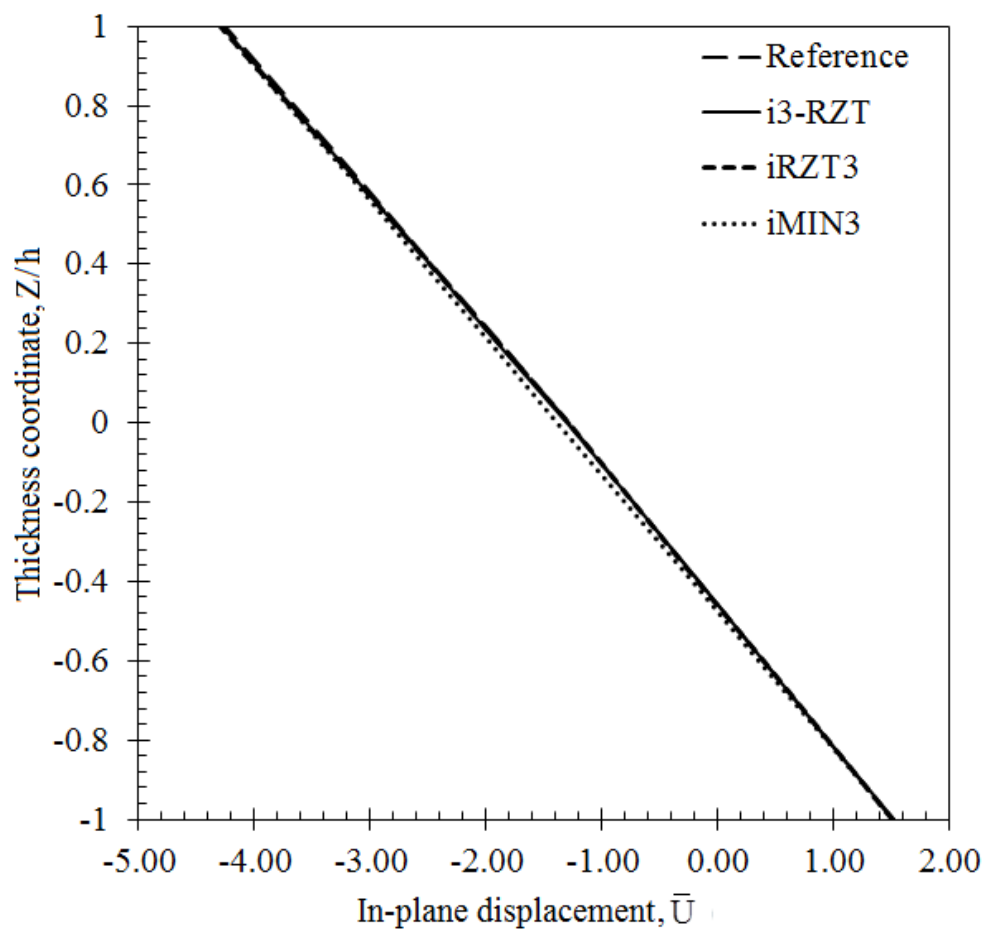

Figure 8 . In-plane displacement $\overline{\mathrm{U}}(0,0.5 \mathrm{~b}, \mathrm{Z})$ variation through the thickness of laminate I. 


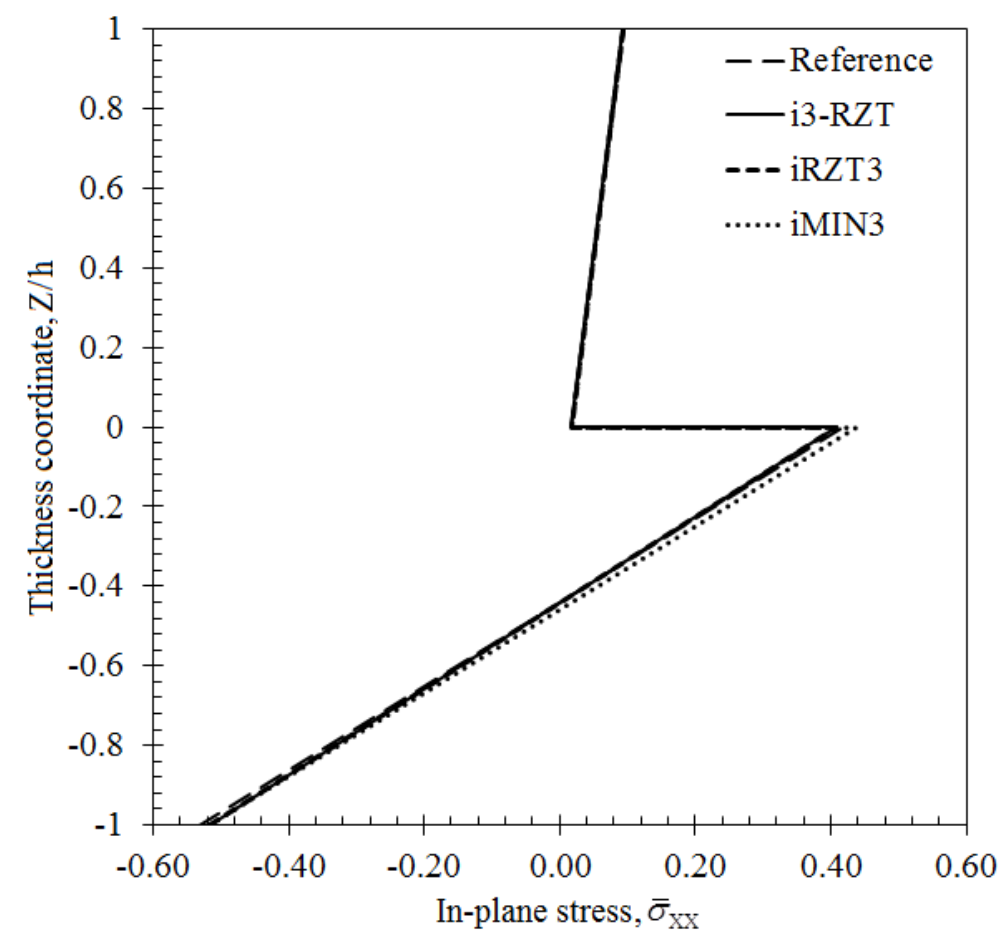

Figure 9. In-plane stress $\bar{\sigma}_{\mathrm{xx}}(0.55 \mathrm{a}, 0.6 \mathrm{~b}, \mathrm{Z})$ variation through the thickness of laminate I.

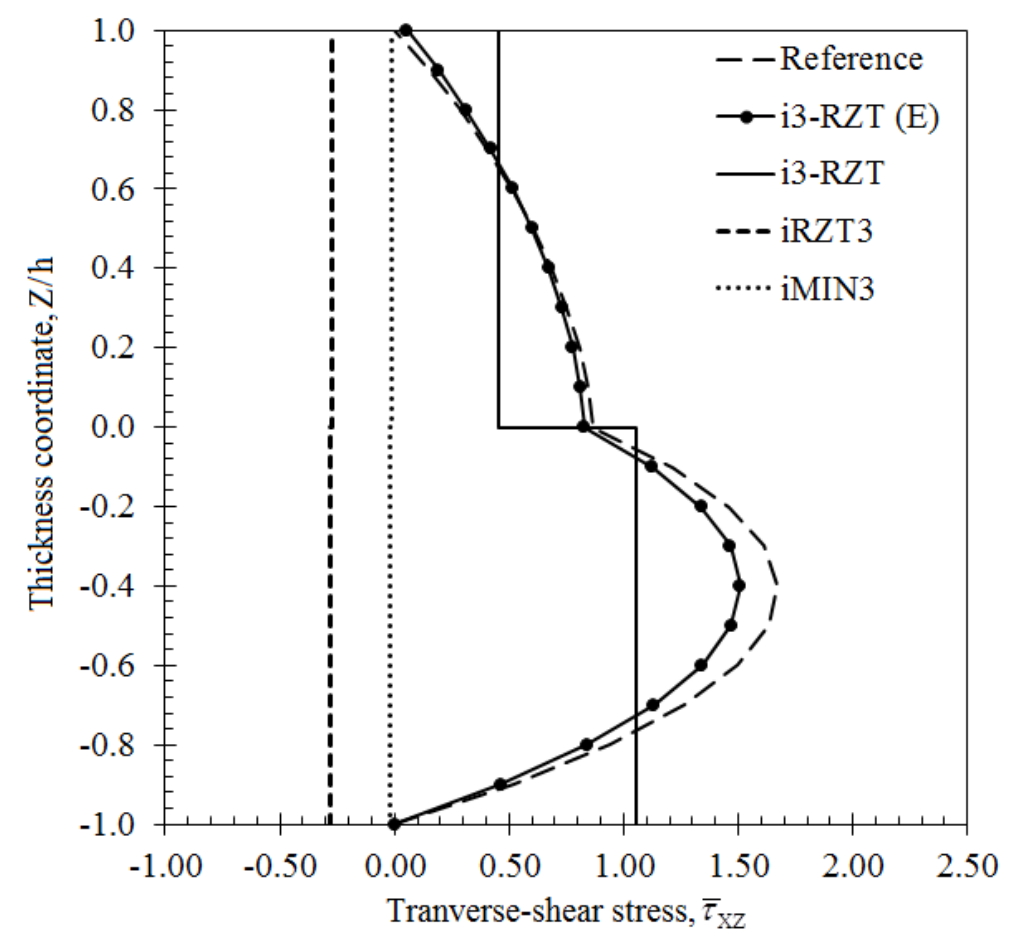

Figure 10. Transverse-shear stress $\bar{\tau}_{\mathrm{XZ}}(0.11 \mathrm{a}, 0.2 \mathrm{~b}, \mathrm{Z})$ variation through the thickness of laminate I. 


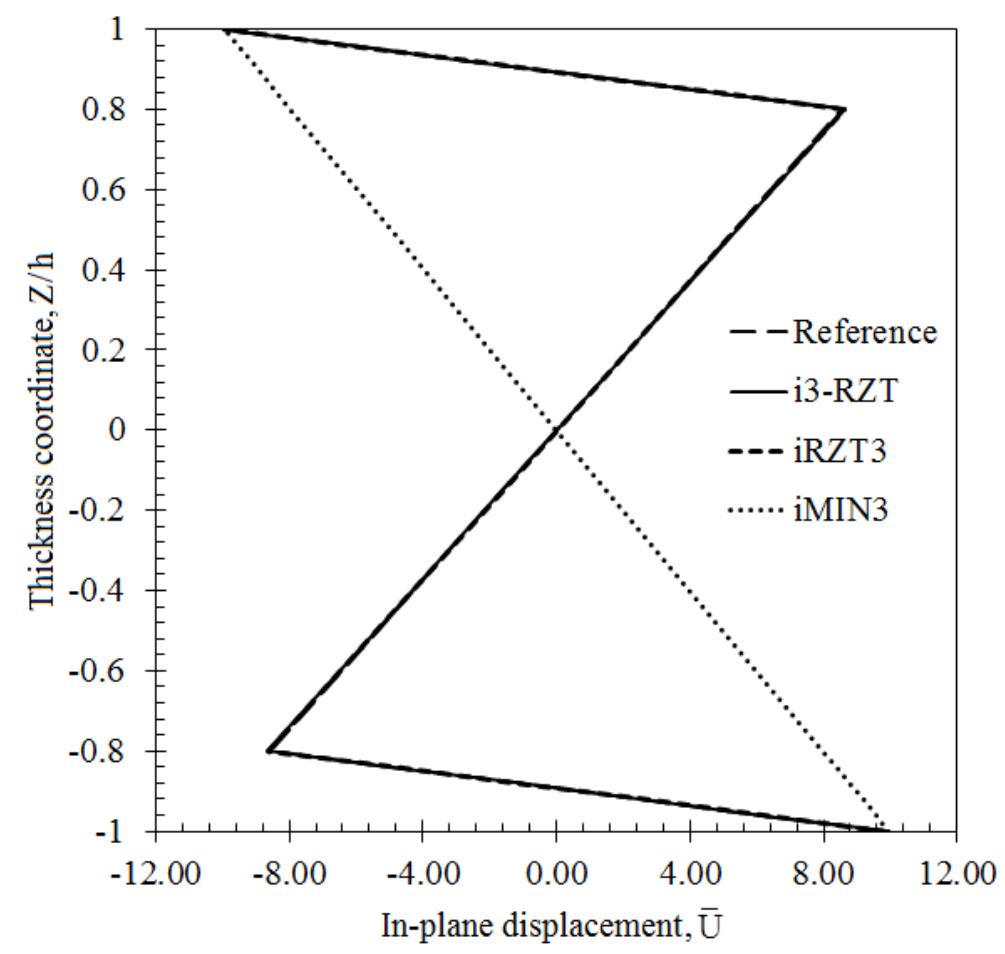

Figure 11. In-plane displacement $\bar{U}(0,0.5 b, Z)$ variation through the thickness of laminate II.

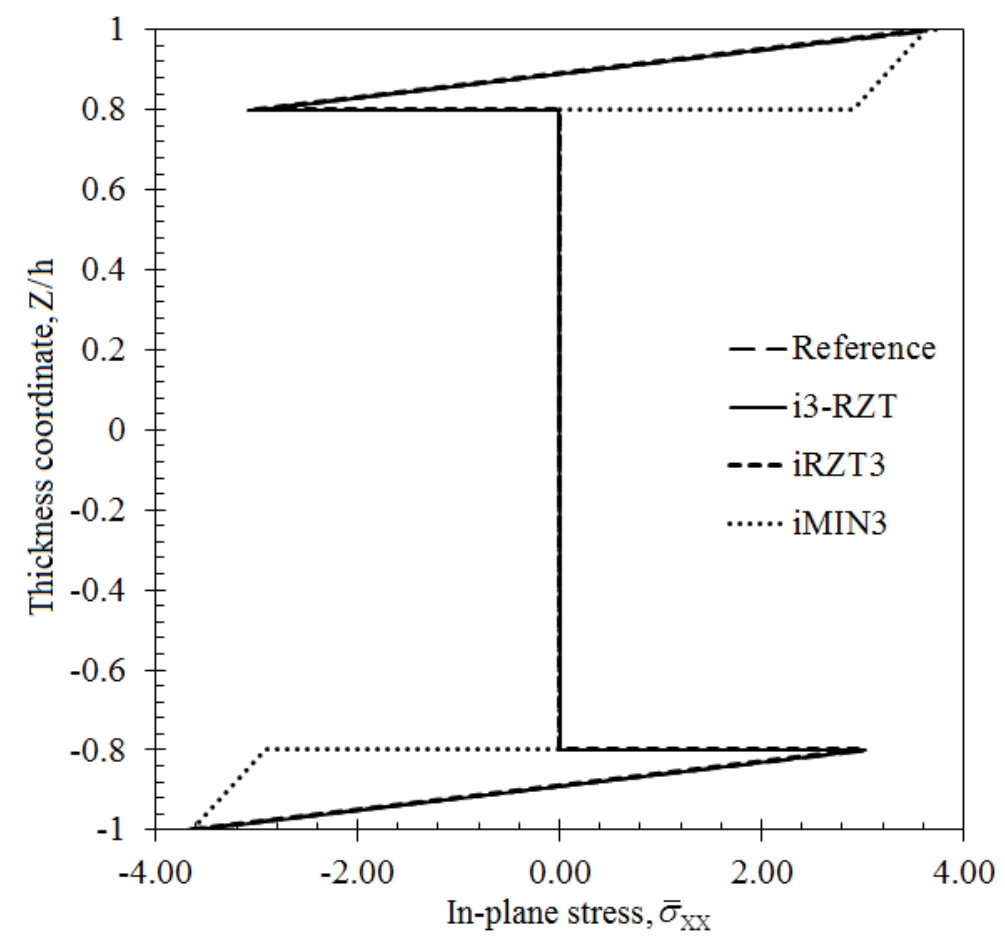

Figure 12. In-plane stress $\bar{\sigma}_{\mathrm{xx}}(0.55 \mathrm{a}, 0.6 \mathrm{~b}, \mathrm{Z})$ variation through the thickness of laminate II. 


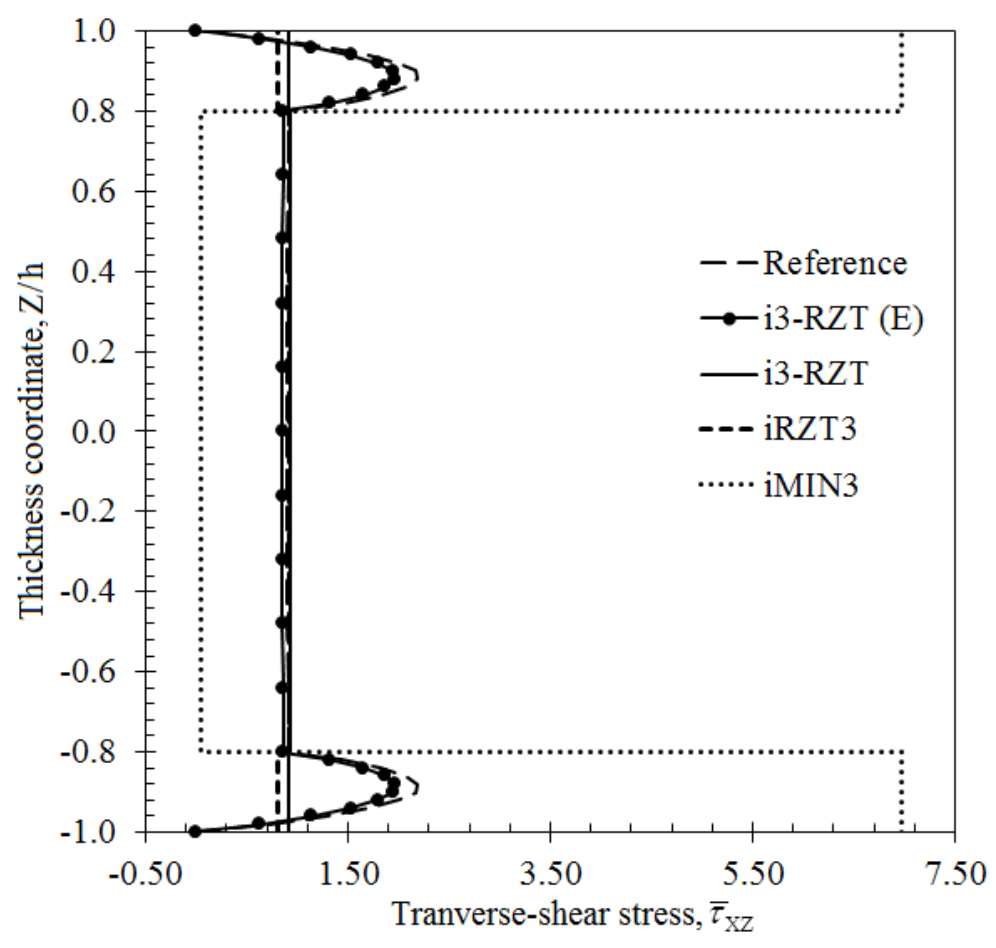

Figure 13. Transverse-shear stress $\bar{\tau}_{\mathrm{xZ}}(0.11 \mathrm{a}, 0.2 \mathrm{~b}, \mathrm{Z})$ variation through the thickness of laminate II.

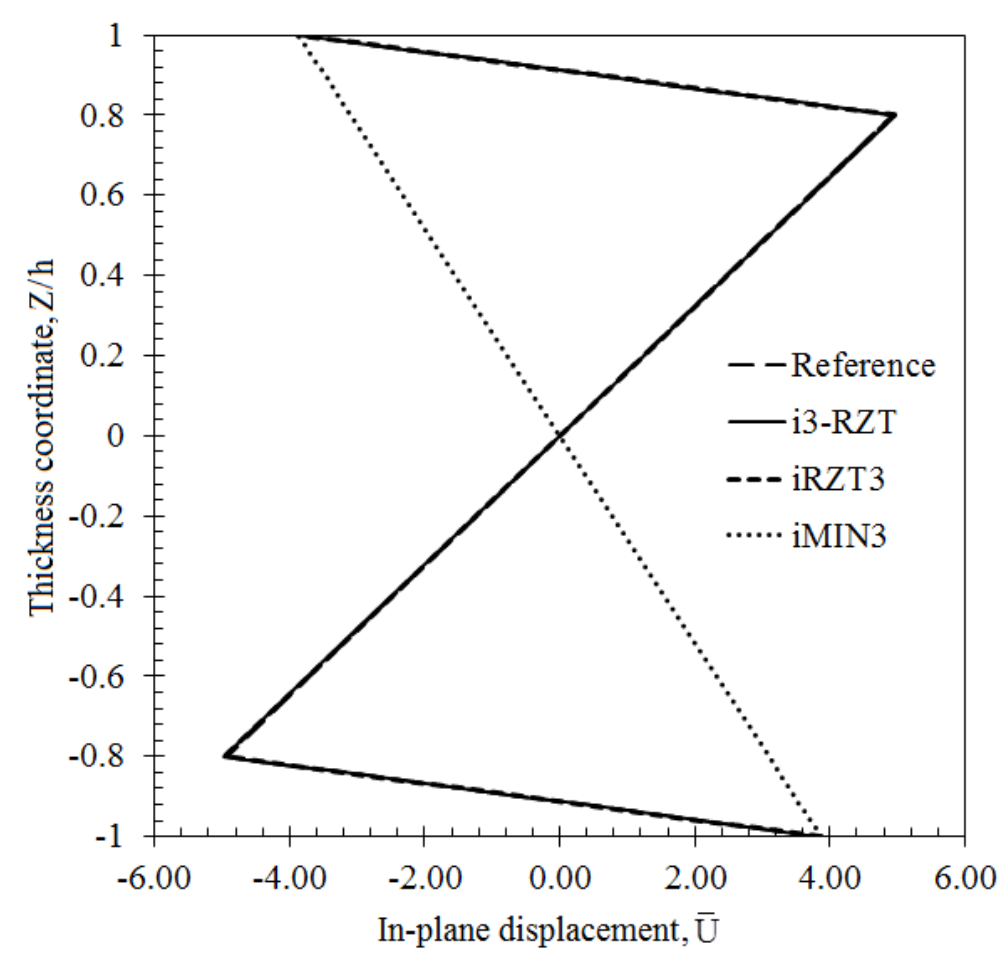

Figure 14. In-plane displacement $\bar{U}(0,0.5 b, Z)$ variation through the thickness of laminate III. 


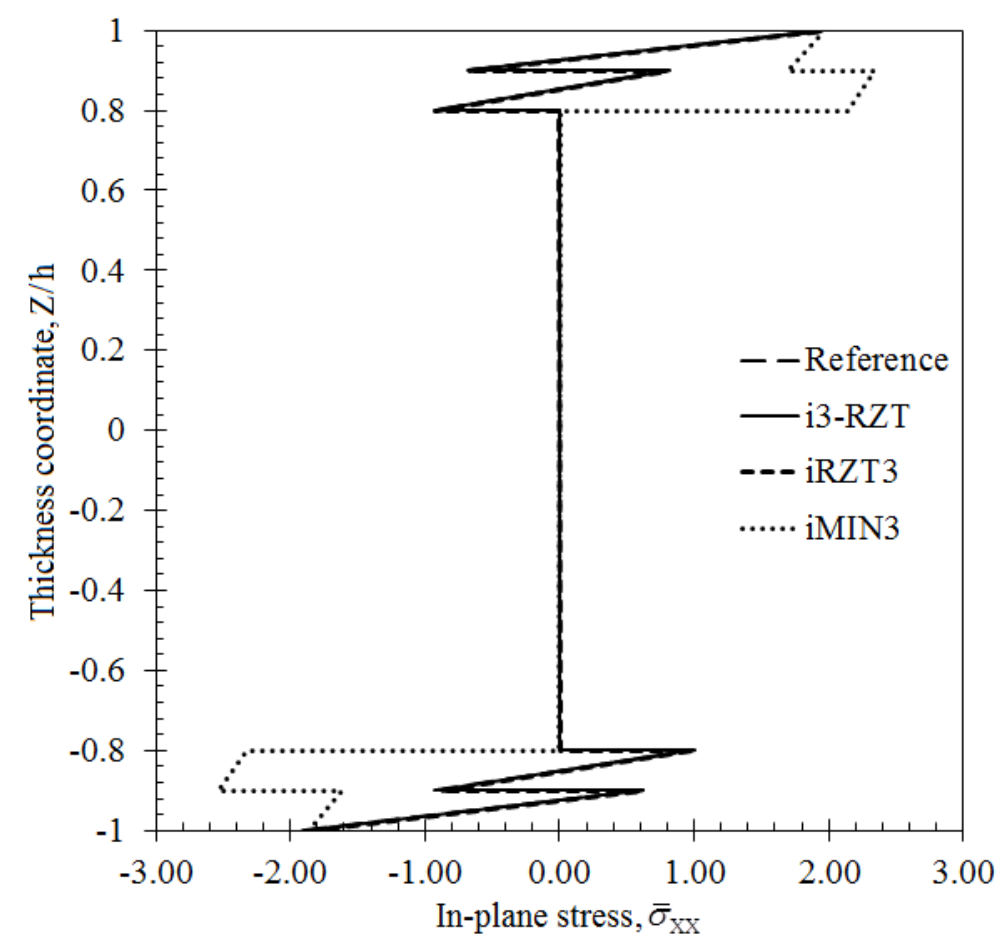

Figure 15. In-plane stress $\bar{\sigma}_{\mathrm{XX}}(0.55 \mathrm{a}, 0.6 \mathrm{~b}, \mathrm{Z})$ variation through the thickness of laminate III.

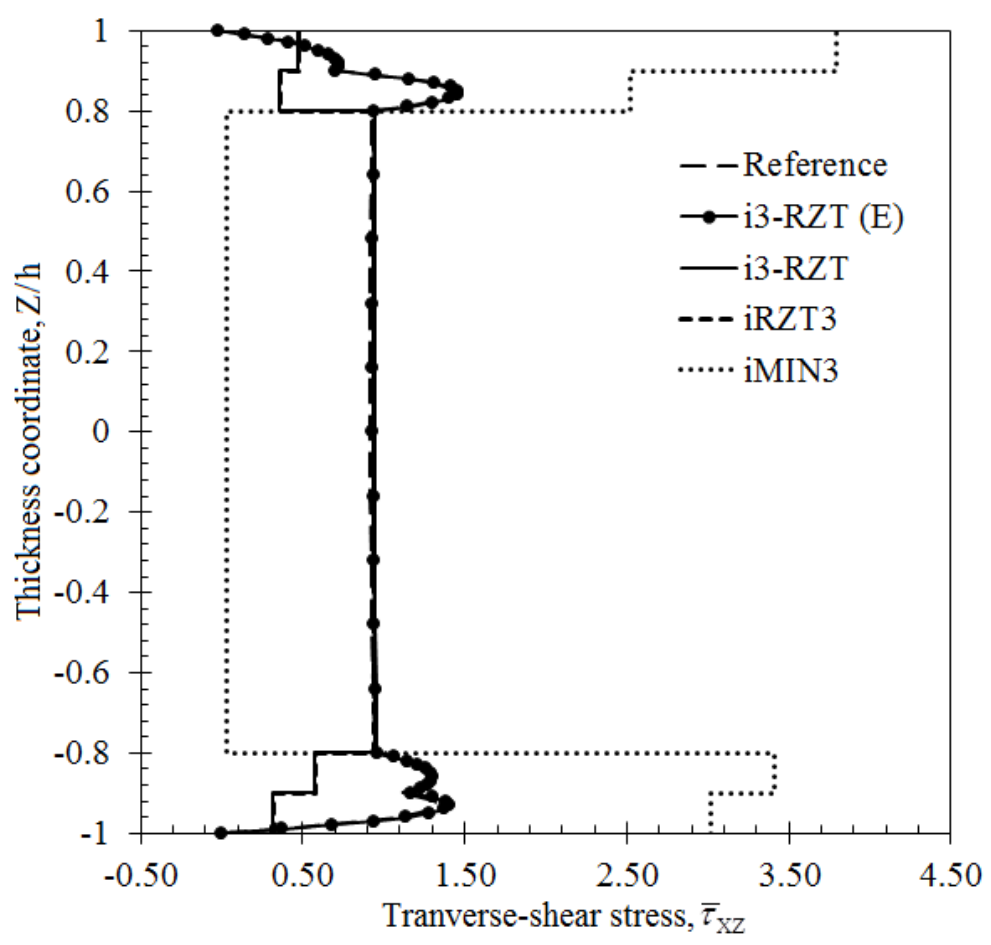

Figure 16. Transverse-shear stress $\bar{\tau}_{\mathrm{xZ}}(0.11 \mathrm{a}, 0.2 \mathrm{~b}, \mathrm{Z})$ through the thickness of laminate III. 


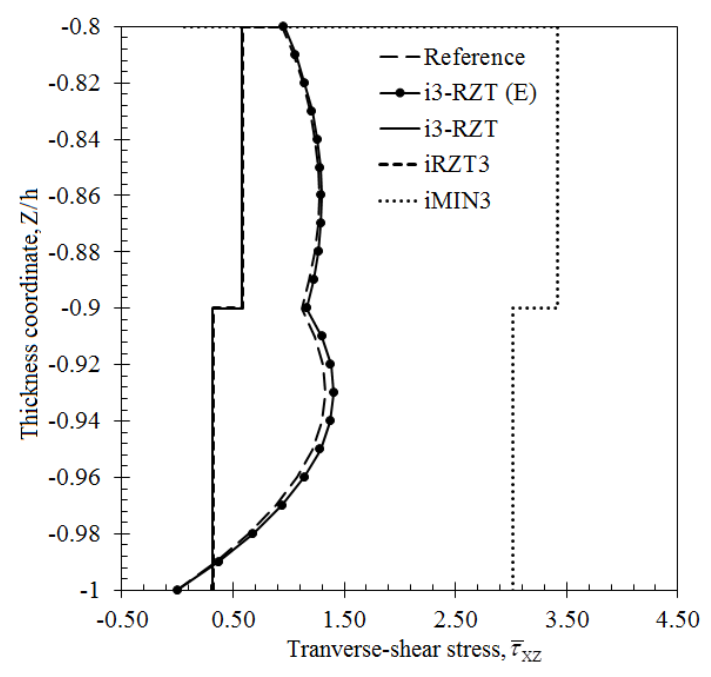

(a)

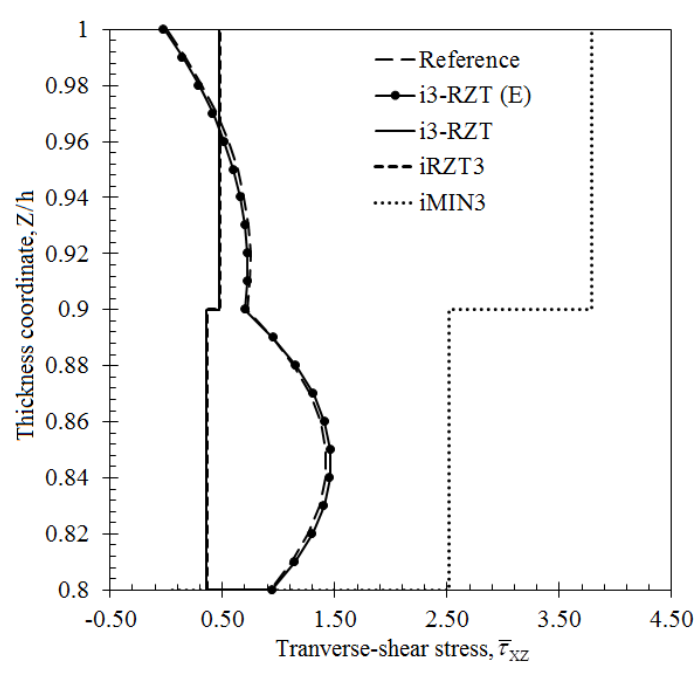

(b)

Figure 17. Zoomed view of Figure 16: (a) Thickness coordinate, $\mathrm{Z} / \mathrm{h} \in[-0.8,-1]$; (b) Thickness coordinate, $\mathrm{Z} / \mathrm{h} \in[0.8,1]$.

Furthermore, in Figures 18-23, contour plots of the $\overline{\mathrm{W}}, \bar{\theta}_{\mathrm{X}}, \bar{\theta}_{\mathrm{Y}}, \bar{\psi}_{\mathrm{X}}, \bar{\psi}_{\mathrm{Y}}$, and $\bar{\sigma}_{\mathrm{v}}$ variables are compared between iFEM/i3-RZT and high-fidelity FEM analyses. For conciseness of the paper, only contour plots of the laminate III (i.e., the most complex laminate in comparison to others) is included. As can be seen from all these figures, both iFEM and FEM contours are graphically indistinguishable, confirming the superior bending predictions of the new i3-RZT element.
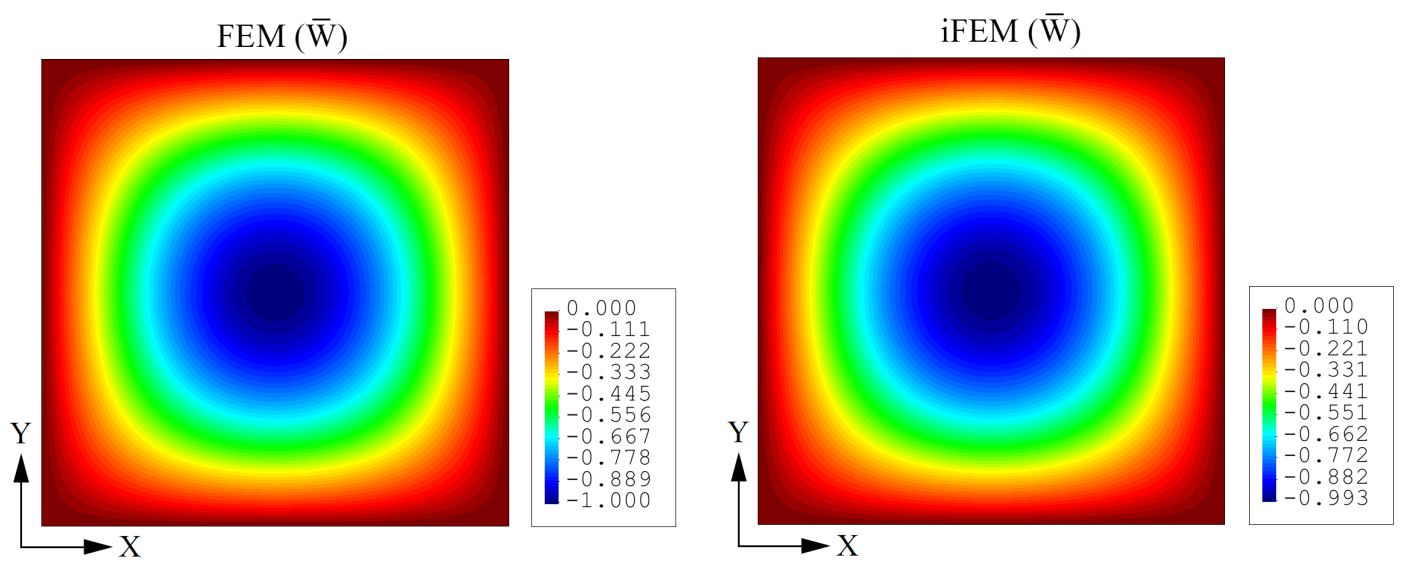

Figure 18. Contour plots of $\overline{\mathrm{W}}$ displacement for laminate III: Comparison between high-fidelity FEM and iFEM $\left(\mathrm{n}^{\mathrm{e}}=4\right)$ analyses. 
$\operatorname{FEM}\left(\bar{\theta}_{\mathrm{X}}\right)$

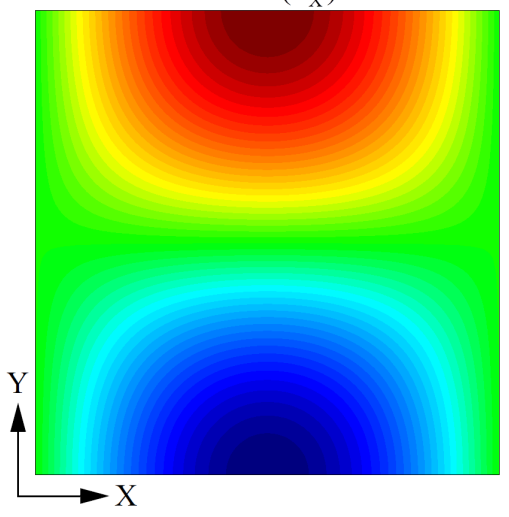

$\operatorname{iFEM}\left(\bar{\theta}_{\mathrm{X}}\right)$

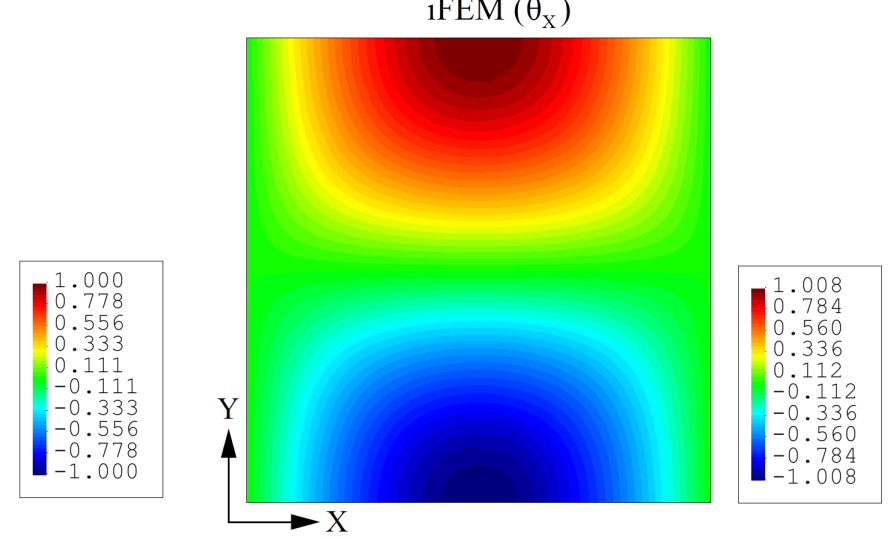

Figure 19. Contour plots of $\bar{\theta}_{\mathrm{X}}$ bending rotation for laminate III: Comparison between high-fidelity FEM and iFEM $\left(\mathrm{n}^{\mathrm{e}}=4\right)$ analyses.
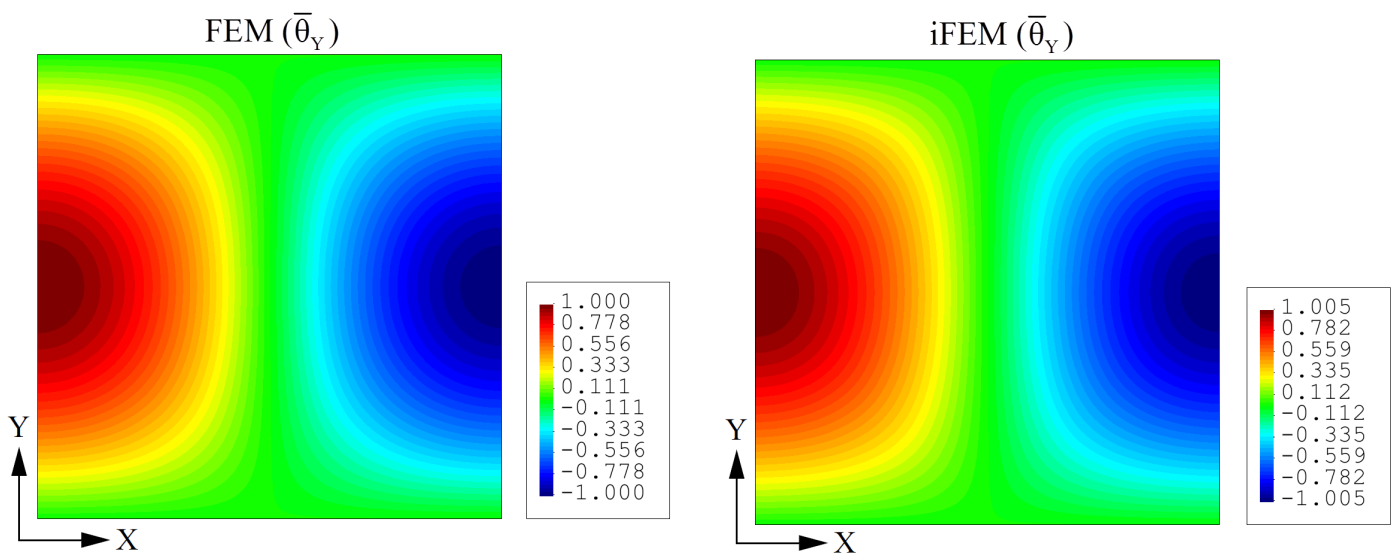

Figure 20. Contour plots of $\bar{\theta}_{\mathrm{Y}}$ bending rotation for laminate III: Comparison between high-fidelity FEM and iFEM $\left(\mathrm{n}^{\mathrm{e}}=4\right)$ analyses.
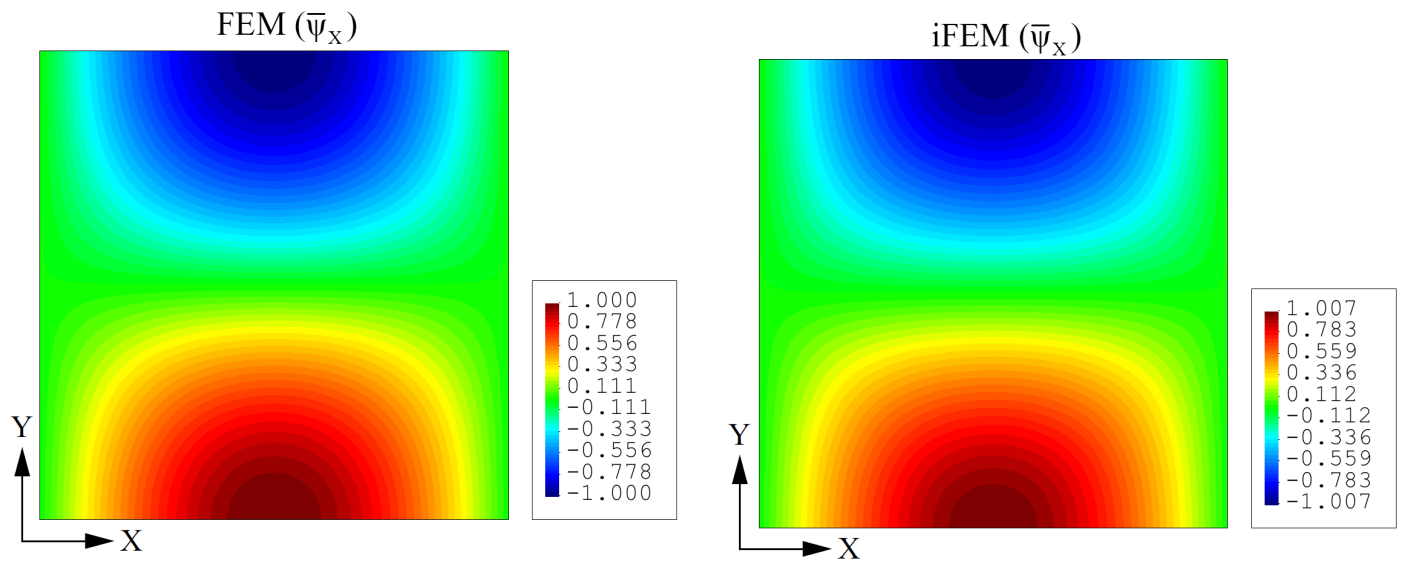

Figure 21. Contour plots of $\bar{\psi}_{\mathrm{X}}$ zigzag rotation for laminate III: Comparison between high-fidelity FEM and iFEM $\left(n^{\mathrm{e}}=4\right)$ analyses. 


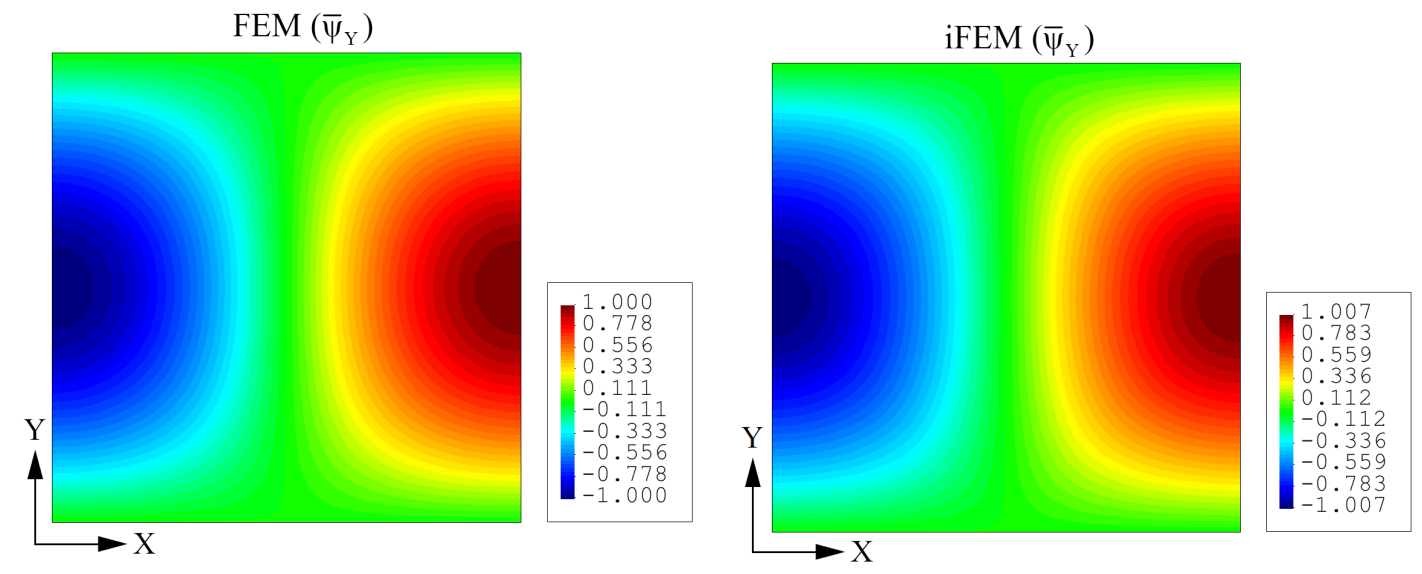

Figure 22. Contour plots of $\bar{\psi}_{\mathrm{Y}}$ zigzag rotation for laminate III: Comparison between high-fidelity FEM and iFEM $\left(\mathrm{n}^{\mathrm{e}}=4\right)$ analyses.
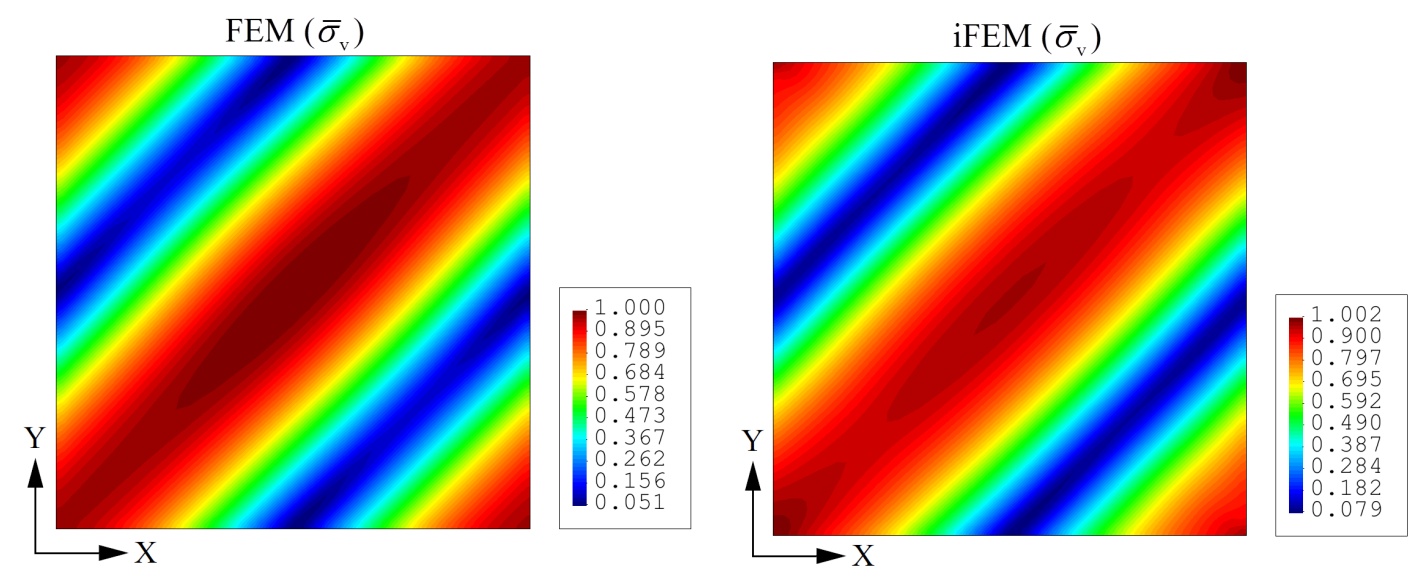

Figure 23. Contour plots of $\bar{\sigma}_{\mathrm{v}}$ von Mises stress at thickness coordinate $\mathrm{Z} / \mathrm{h}=-1$ of laminate III: Comparison between high-fidelity FEM and iFEM $\left(n^{\mathrm{e}}=4\right)$ analyses.

\subsection{A wedge structure with a hole}

A wedge structure with a hole near one of the clamped ends is analyzed to demonstrate the applicability of the improved iFEM formulation to more complex engineering structures. As depicted in Figure 24, the wedge structure has overall length of $\mathrm{L}_{1}=6 \mathrm{~m}$, width of $\mathrm{L}_{2}=2 \mathrm{~m}$, and uniform thickness of $2 \mathrm{~h}=0.2 \mathrm{~m}$. The wedge structure is composed of panels $\mathrm{A}$ and $\mathrm{B}$, each of which has an element group coordinate system, i.e., $\left(\mathrm{X}_{\alpha}, \mathrm{Y}_{\alpha}, \mathrm{Z}_{\alpha}\right)(\alpha=\mathrm{A}, \mathrm{B})$. As presented in Figure 25 , the kinematic variables $\left(\mathrm{U}_{\alpha}, \mathrm{V}_{\alpha}, \mathrm{W}_{\alpha}\right)(\alpha=\mathrm{A}, \mathrm{B})$ represent the translations along the positive coordinate $\left(\mathrm{X}_{\alpha}, \mathrm{Y}_{\alpha}, \mathrm{Z}_{\alpha}\right)(\alpha=\mathrm{A}, \mathrm{B})$ directions, respectively, whereas the 
kinematic variables $\left(\theta_{\mathrm{X} \alpha}, \theta_{\mathrm{Y} \alpha}\right)(\alpha=\mathrm{A}, \mathrm{B})$ and $\left(\psi_{\mathrm{X} \alpha}, \psi_{\mathrm{Y} \alpha}\right)(\alpha=\mathrm{A}, \mathrm{B})$ denote bending and zigzag rotations around the positive $\left(\mathrm{X}_{\alpha}, \mathrm{Y}_{\alpha}\right)(\alpha=\mathrm{A}, \mathrm{B})$ directions, respectively. Both ends of the wedge are clamped, and the clamped boundary conditions along $X_{A}=X_{B}=0 m$ and $X_{A}=X_{B}=6 m$ are specified as

$$
\mathrm{U}_{\alpha}=\mathrm{V}_{\alpha}=\mathrm{W}_{\alpha}=\theta_{\beta \alpha}=\psi_{\beta \alpha}=0 \quad(\alpha=\mathrm{A}, \mathrm{B} ; \beta=\mathrm{X}, \mathrm{Y})
$$

A body force of $\mathrm{g}=100 \mathrm{kN} / \mathrm{m}^{3}$ is applied to the wedge structure along the negative $\mathrm{Z}_{\mathrm{A}}$ direction as shown in Figure 24. A five-layer, cross-ply sandwich laminate with carbon-epoxy face sheets and a thick PVC core is considered to represent moderately thin sandwich plates with a span-to-thickness ratio of $\mathrm{L}_{1} / 2 \mathrm{~h}=30$. The stacking sequences of the wedge laminate are listed in Table 12.

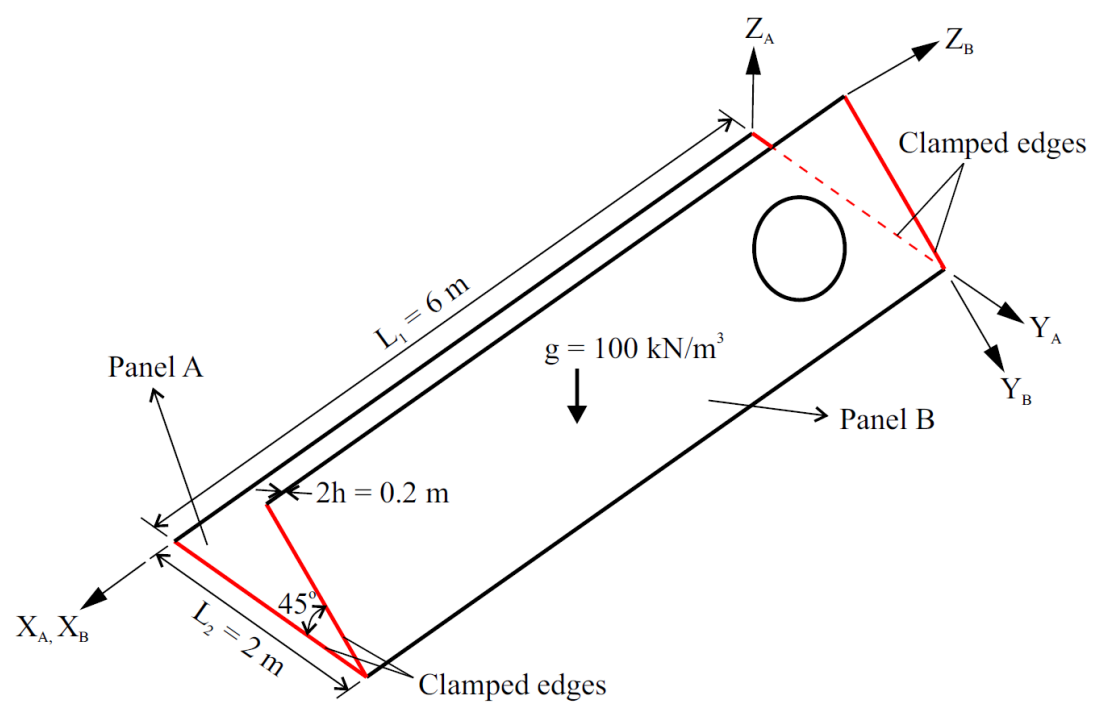

Figure 24. Isometric view of the wedge structure. 


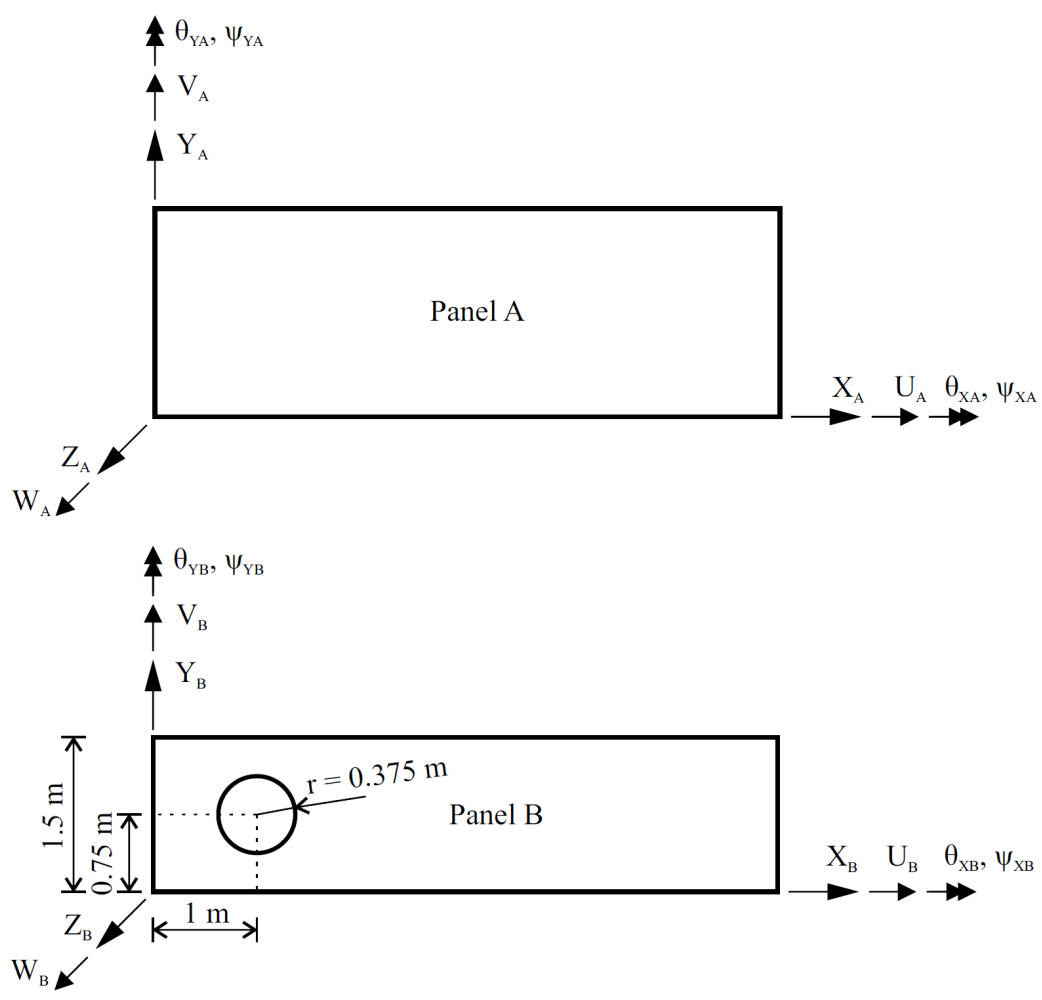

Figure 25. Panels A and B, group coordinate systems, and kinematic variables.

Table 12. Laminate stacking sequence of the wedge structure (in the positive $Z_{A}$ and $\mathrm{Z}_{\mathrm{B}}$ directions).

\begin{tabular}{llll}
\hline Wedge Laminate & $\begin{array}{l}\text { Normalized lamina } \\
\text { thickness, } \mathrm{h}^{(k)} / \mathrm{h}\end{array}$ & $\begin{array}{l}\text { Lamina } \\
\text { materials }\end{array}$ & $\begin{array}{l}\text { Lamina } \\
\text { orientation }\left[{ }^{\circ}\right]\end{array}$ \\
\hline Cross-ply sandwich & $(0.05 / 0.05 / 0.8 / 0.05 / 0.05)$ & $(\mathrm{C} / \mathrm{C} / \mathrm{P} / \mathrm{C} / \mathrm{C})$ & $(0 / 90 / 0 / 90 / 0)$ \\
\hline
\end{tabular}

An RZT-based FEM convergence study was performed to establish an accurate reference solution for this problem. As depicted in Figure 26, the highest fidelity FEM mesh consisted of 18802 randomly distributed triangular elements with an edge size of $\mathrm{e}^{\text {size }}=0.05 \mathrm{~m}$ and 86535 DOF. The FEM deflections and rotations are used to compute the simulated strain-sensor strains. For panels $\mathrm{A}$ and $\mathrm{B}$, the maximum displacements, von Mises stresses, bending and zigzag rotations obtained from FEM analysis are listed in Tables 13-14, respectively. To assess the accuracy of the displacement, rotation, and stress responses, it would be convenient to use these reference values as normalization factors. 


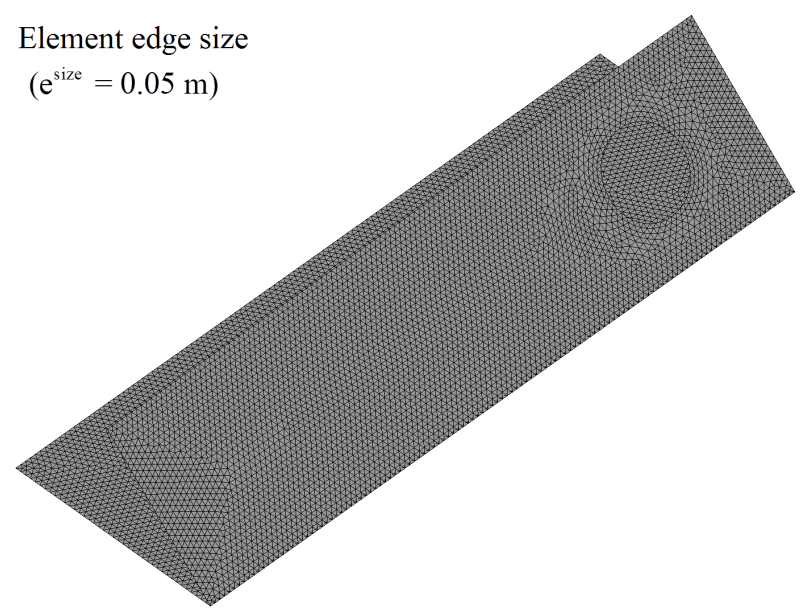

Figure 26. Discretization of the wedge structure using 18802 elements.

Table 13. Maximum displacements and von Mises stresses of the wedge panels.

\begin{tabular}{lllll}
\hline$\alpha$ & $\mathrm{U}_{\alpha, \max }^{\mathrm{FEM}}[\mathrm{m}]$ & $\mathrm{V}_{\alpha, \max }^{\mathrm{FEM}}[\mathrm{m}]$ & $\mathrm{W}_{\alpha, \max }^{\mathrm{FEM}}[\mathrm{m}]$ & $\sigma_{\mathrm{v} \alpha, \max }^{\mathrm{FEM}}[\mathrm{Pa}]$ \\
\hline $\mathrm{A}$ & $-4.353 \times 10^{-5}$ & $-2.059 \times 10^{-4}$ & $-1.389 \times 10^{-2}$ & $9.874 \times 10^{7}$ \\
$\mathrm{~B}$ & $1.101 \times 10^{-4}$ & $8.601 \times 10^{-4}$ & $-1.031 \times 10^{-2}$ & $9.238 \times 10^{7}$ \\
\hline
\end{tabular}

Table 14. Maximum bending and zigzag rotations of the wedge panels.

\begin{tabular}{lllll}
\hline$\alpha$ & $\theta_{\mathrm{X} \alpha, \max }^{\mathrm{FEM}}[\mathrm{rad}]$ & $\theta_{\mathrm{Y} \alpha, \max }^{\mathrm{FEM}}[\mathrm{rad}]$ & $\psi_{\mathrm{X} \alpha, \max }^{\mathrm{FEM}}[\mathrm{rad}]$ & $\psi_{\mathrm{Y} \alpha, \max }^{\mathrm{FEM}}[\mathrm{rad}]$ \\
\hline $\mathrm{A}$ & $6.119 \times 10^{-3}$ & $1.243 \times 10^{-3}$ & $-1.350 \times 10^{-3}$ & $-7.128 \times 10^{-3}$ \\
$\mathrm{~B}$ & $6.084 \times 10^{-3}$ & $1.120 \times 10^{-3}$ & $-2.117 \times 10^{-3}$ & $5.314 \times 10^{-3}$ \\
\hline
\end{tabular}

Five different iFEM analyses of the wedge structure were performed using five different networks of strain rosettes. Each iFEM analysis refers to a case study number; e.g., the first analysis is called "iFEM (Case I)" and the third analysis is called "iFEM (Case III)." Through the thickness coordinate, three strain rosettes are located at the centroid of each element for all iFEM cases; one on the top surface, one on the bottom surface, and one on the nearest interface to the bottom surface of the laminate. In contrast to example presented in Section 4.1, the discrete strain measures were not smoothed a priori and they were directly used as input in iFEM (Cases I-V). Using the absolute values of the reference solutions listed in Tables 13-14, the displacements, 
bending and zigzag rotations, and von Mises stresses obtained from both iFEM and FEM analyses are normalized as

$\bar{\chi}_{\alpha}=\chi_{\alpha} /\left|\chi_{\alpha, \max }^{\mathrm{FEM}}\right|\left(\chi=\mathrm{U}, \mathrm{V}, \mathrm{W}, \theta_{\mathrm{X}}, \theta_{\mathrm{Y}}, \psi_{\mathrm{X}}, \psi_{\mathrm{Y}}, \sigma_{\mathrm{v}}\right)$

where $\alpha=\mathrm{A}$ is for panel A and $\alpha=\mathrm{B}$ is for panel $\mathrm{B}$.

In iFEM (Case I), the i3-RZT discretization is identical to high-fidelity mesh used in the FEM analysis. As presented in Figure 27, the i3-RZT model has 18802 uniformly distributed triangular elements, each of which has three strain rosettes $\left(\boldsymbol{\varepsilon}_{i}^{+}, \boldsymbol{\varepsilon}_{i}^{-}, \boldsymbol{\varepsilon}_{i}^{j}\right)$. In iFEM (Case II), the top-surface, bottom-surface, and $j$-th interface strain rosettes are removed from 13678 i3-RZT elements, and the resulting i3-RZT mesh has only $5124 \times 3$ strain rosettes as shown in Figure 28 .

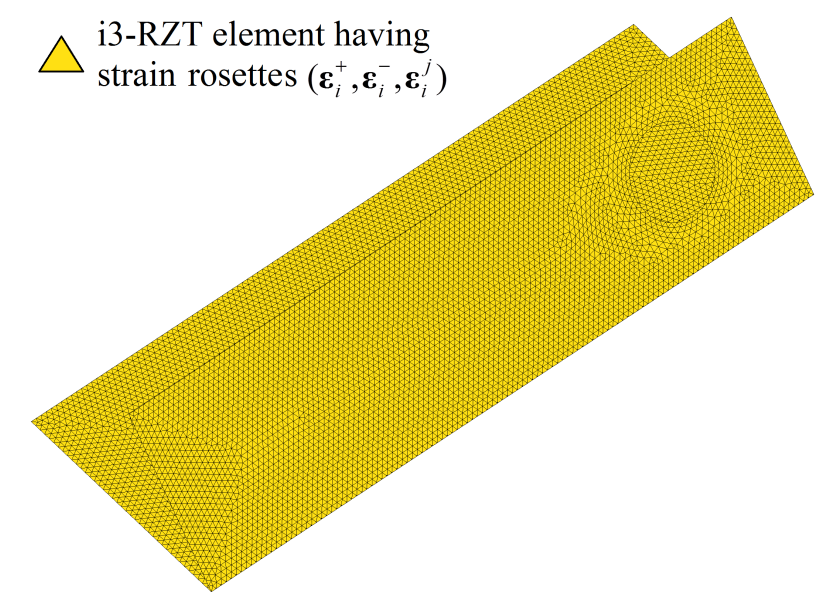

Figure 27. The i3-RZT model used in iFEM (Case I).

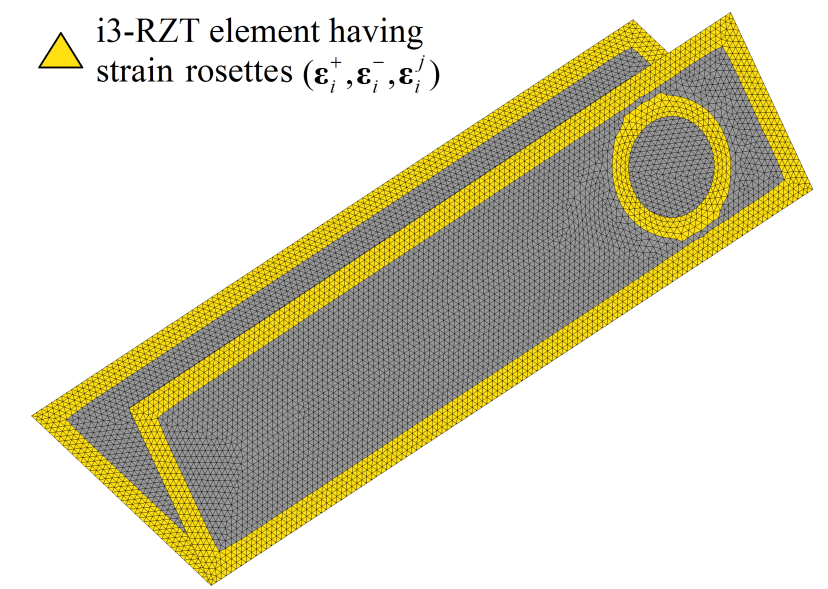

Figure 28. The i3-RZT model used in iFEM (Case II). 


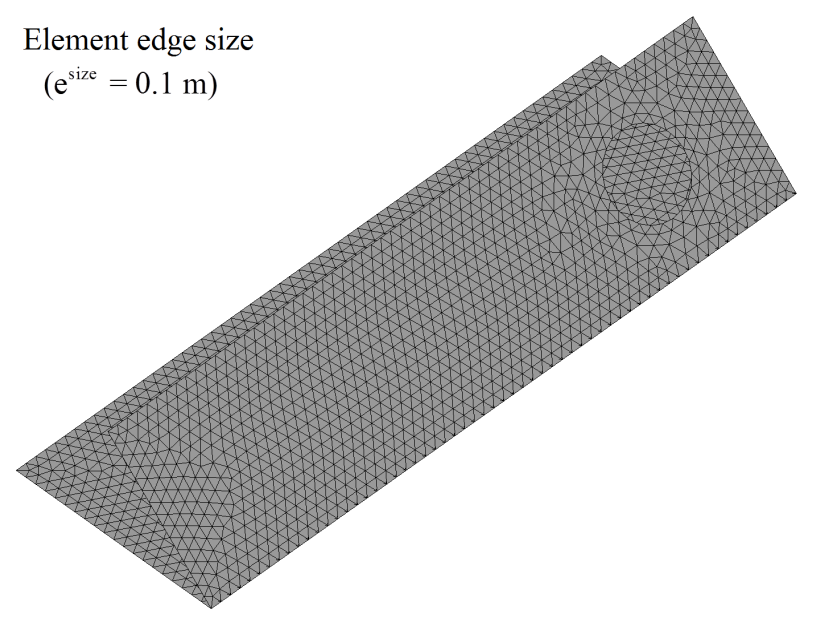

Figure 29. Discretization of the wedge structure using 4644 elements.

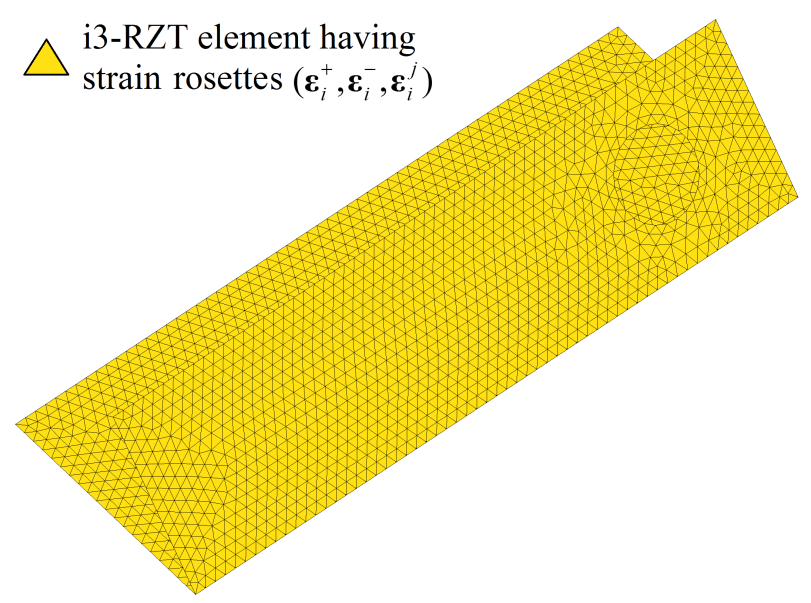

Figure 30. The i3-RZT model used in iFEM (Case III).

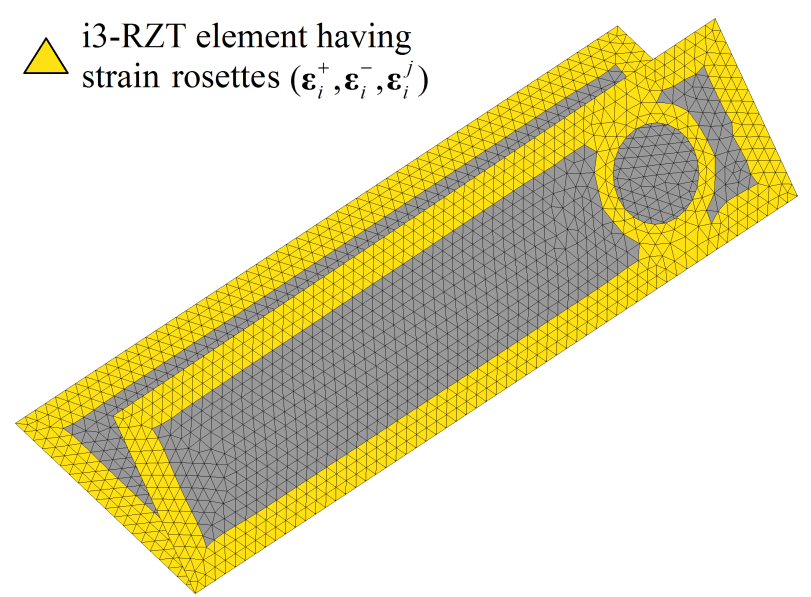

Figure 31. The i3-RZT model used in iFEM (Case IV). 


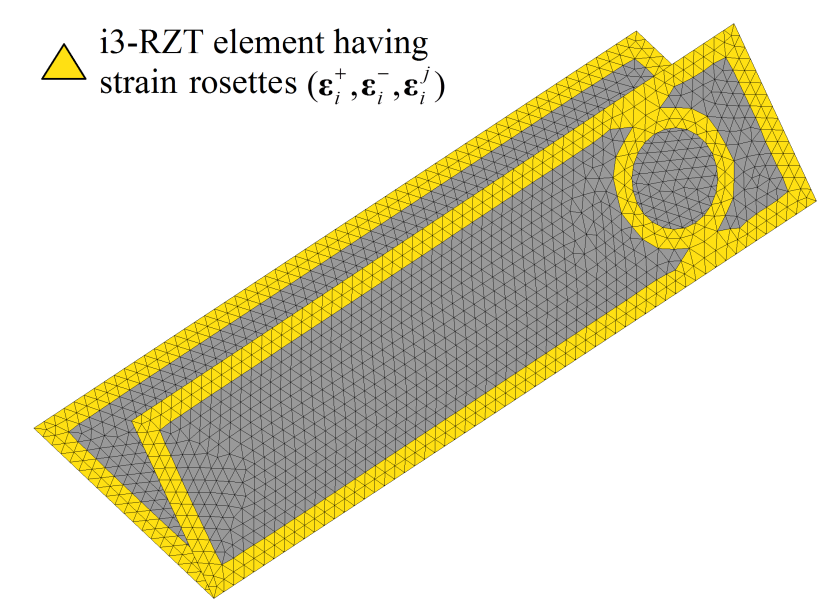

Figure 32. The i3-RZT model used in iFEM (Case V).

A coarser i3-RZT discretization is used in the last three case studies, iFEM (Cases IIIV). As depicted in Figure 29, the coarser i3-RZT discretization consisted of 4644 randomly distributed triangular elements with an edge size of $\mathrm{e}^{\text {size }}=0.1 \mathrm{~m}$ and 21861 DOF. In iFEM (Case III), as presented in Figure 30, the i3-RZT model has 4644 inverse-elements each of which has three strain rosettes $\left(\boldsymbol{\varepsilon}_{i}^{+}, \boldsymbol{\varepsilon}_{i}^{-}, \boldsymbol{\varepsilon}_{i}^{j}\right)$. In iFEM (Case IV), the top-surface, bottom-surface, and $j$-th interface strain rosettes are removed from 2839 i3-RZT elements, and the resulting i3-RZT mesh has only $1825 \times 3$ strain rosettes (refer to Figure 31). In iFEM (Case V), as shown in Figure 32, the i3-RZT model has only 1288 inverse-elements installed with three strain rosettes $\left(\boldsymbol{\varepsilon}_{i}^{+}, \boldsymbol{\varepsilon}_{i}^{-}, \boldsymbol{\varepsilon}_{i}^{j}\right)$, and the remaining 3376 i3-RZT elements has no in situ strain components. For an i3-RZT element that has no in situ strain components, the corresponding weighting coefficients are set to $10^{-5}$. Moreover, the weighting constants corresponding to the transverseshear strain measures are set to a small value as $w_{10}=w_{11}=10^{-8}$ and $w_{12}=w_{13}=10^{-6}$ for iFEM (Cases I-V).

The percent difference between iFEM (Cases I-V) and FEM predictions for the maximum values of normalized expressions given in Eq. (40) are listed for panels A and B in Tables 15-16, respectively. As can be seen from these tables, iFEM (Case I) and FEM predictions are almost the same for all the variables. These results clearly demonstrate the superior accuracy of the $\mathrm{iFEM} / \mathrm{i3}-\mathrm{RZT}$ solutions when a high-fidelity i3-RZT model in which all elements have in situ strain measurements is used. Moreover, the percent differences between iFEM (Case II) and FEM estimates for all 
of the variables are less than $4 \%$ and $2 \%$ for panels $A$ and B, respectively. These results confirm the superior membrane-bending coupled predictions of the i3-RZT element, especially considering a high-fidelity i3-RZT model in which relatively few elements have in situ strain measurements.

In addition, the percent differences between iFEM (Case III) and FEM predictions for displacement and rotation variables are not more than $5.6 \%$ and $10.8 \%$ for panel $\mathrm{A}$ and $1.1 \%$ and $5.9 \%$ for panel $\mathrm{B}$, respectively. These results demonstrate the high accuracy of the iFEM/i3-RZT capability for shape sensing even when relatively coarse discretization is used. Furthermore, iFEM (Case III) solutions for von Mises stresses differ from the FEM solutions by approximately $14 \%$ for panel A and $15.5 \%$ for panel B. Even though these results might be acceptable for some practical applications, they clearly demonstrate that $\mathrm{iFEM} / \mathrm{i3}-\mathrm{RZT}$ formulation needs a higher fidelity mesh to compute high strain gradients to obtain improved stresses.

Table 15. Percent difference between the iFEM (Cases I-V) and FEM predictions for maximum values of the variables (panel A).

\begin{tabular}{lllllllll}
\hline \multirow{2}{*}{ Case } & \multicolumn{8}{c}{ Percent difference } \\
\cline { 2 - 9 } & $\overline{\mathrm{U}}_{\mathrm{A}}$ & $\overline{\mathrm{V}}_{\mathrm{A}}$ & $\overline{\mathrm{W}}_{\mathrm{A}}$ & $\bar{\theta}_{\mathrm{XA}}$ & $\bar{\theta}_{\mathrm{YA}}$ & $\bar{\psi}_{\mathrm{XA}}$ & $\bar{\psi}_{\mathrm{YA}}$ & $\bar{\sigma}_{\mathrm{vA}}$ \\
\hline $\mathrm{I}$ & 0.0 & 0.0 & 0.0 & 0.0 & 0.0 & 0.1 & 0.0 & 0.2 \\
$\mathrm{II}$ & 0.1 & 0.0 & 0.0 & 1.1 & 3.7 & 2.8 & 0.0 & 0.2 \\
$\mathrm{III}$ & 5.6 & 0.8 & 1.0 & 0.8 & 0.8 & 10.8 & 1.7 & 14.0 \\
$\mathrm{IV}$ & 12.4 & 22.5 & 2.3 & 9.0 & 3.3 & 7.6 & 3.8 & 14.6 \\
$\mathrm{~V}$ & 18.1 & 52.2 & 3.8 & 19.4 & 7.7 & 3.2 & 4.7 & 14.5 \\
\hline
\end{tabular}

Table 16. Percent difference between the iFEM (Cases I-V) and FEM predictions for maximum values of the variables (panel B).

\begin{tabular}{lcccccccc}
\hline \multirow{2}{*}{ Case } & \multicolumn{8}{c}{ Percent difference } \\
\cline { 2 - 9 } & $\overline{\mathrm{U}}_{\mathrm{B}}$ & $\overline{\mathrm{V}}_{\mathrm{B}}$ & $\overline{\mathrm{W}}_{\mathrm{B}}$ & $\bar{\theta}_{\mathrm{XB}}$ & $\bar{\theta}_{\mathrm{YB}}$ & $\bar{\psi}_{\mathrm{XB}}$ & $\bar{\psi}_{\mathrm{YB}}$ & $\bar{\sigma}_{\mathrm{vB}}$ \\
\hline $\mathrm{I}$ & 0.0 & 0.0 & 0.0 & 0.0 & 0.0 & 0.0 & 0.0 & 0.2 \\
$\mathrm{II}$ & 0.2 & 0.3 & 0.0 & 1.1 & 0.1 & 1.7 & 0.0 & 0.2 \\
$\mathrm{III}$ & 1.1 & 0.7 & 1.1 & 0.8 & 5.9 & 5.7 & 2.4 & 15.5 \\
$\mathrm{IV}$ & 3.6 & 0.3 & 2.0 & 9.1 & 8.5 & 6.4 & 5.0 & 15.5 \\
$\mathrm{~V}$ & 5.1 & 2.5 & 4.9 & 19.5 & 21.5 & 8.5 & 5.8 & 15.5 \\
\hline
\end{tabular}


As can be seen from Table 13, the maximum deflections, $\mathrm{W}_{\alpha, \max }^{\mathrm{FEM}}(\alpha=\mathrm{A}, \mathrm{B})$, are much greater than the maximum in-plane displacements and/or rotations, thus the deformed shape (total deformation) of the wedge structure is mainly caused by the deflections. Hence, the accuracy of monitoring the deflections $\overline{\mathrm{W}}_{\mathrm{A}}$ and $\overline{\mathrm{W}}_{\mathrm{B}}$ is crucial for monitoring total deformation. The percent differences between iFEM (Case IV) and FEM estimates for $\overline{\mathrm{W}}_{\mathrm{A}}$ and $\overline{\mathrm{W}}_{\mathrm{B}}$ are only $2.3 \%$ and $2.0 \%$, whereas iFEM (Case V) predictions for $\overline{\mathrm{W}}_{\mathrm{A}}$ and $\overline{\mathrm{W}}_{\mathrm{B}}$ differ from the FEM predictions by only $3.8 \%$ and $4.9 \%$, respectively. These results demonstrate that iFEM predictions remain sufficiently accurate even considering a coarse i3-RZT model with the missing strain rosette data in many elements. Besides, these results remarkably prove the superior capability of the i3-RZT element for shape sensing, even though the percent differences between iFEM (Cases IV-V) for in-plane displacement and rotations are relatively higher. Furthermore, the percent differences between iFEM (Cases IV-V) and FEM predictions for von Mises stresses are not more than $14.6 \%$ and $15.5 \%$ for panels A and $\mathrm{B}$, respectively. These percent differences are very similar to the corresponding percent differences obtained for iFEM (Case III). Hence, these results prove that an i3-RZT model in which few elements have strain rosettes can predict similar maximum stresses in comparison to an i3-RZT model in which all elements have strain rosettes. In addition to the percent difference results, contour plots for the normalized expressions given in Eq. (40) are compared between iFEM (Case IV) and high-fidelity FEM analyses in Figures 33-40. Note that, only contour plots of the iFEM (Case IV), i.e., one of the most challenging case study in comparison to others, is included herein for conciseness of the paper. In Figures 35-37 and 39-40, contour plots for iFEM (Case IV) are graphically almost identical to those of FEM. Moreover, the remaining contour plots for iFEM (Cases IV) are generally in good agreement with the contour plots of FEM (refer to Figures 33, 34, and 38). The iFEM predictions remain sufficiently accurate even with the missing strain rosette data in many elements. Thus, these results demonstrate the predictive capability and practical applicability of the enhanced iFEM formulation for shape and stress sensing of complex composite/sandwich structures exhibiting complicated deformed shapes. 

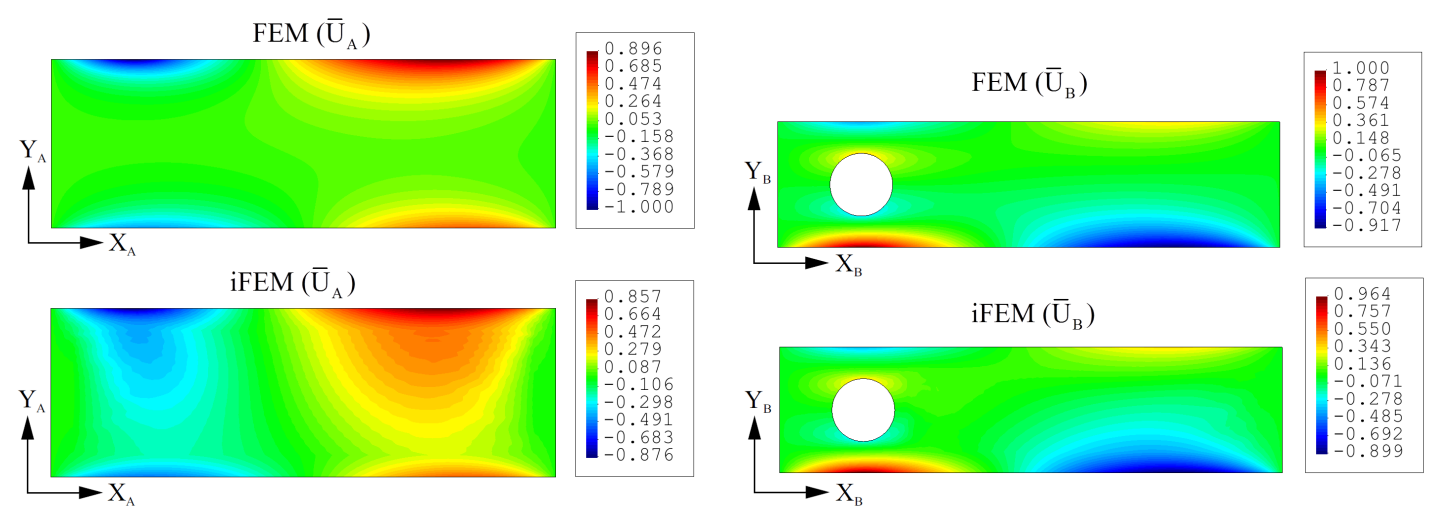

Figure 33. Contour plots of $\overline{\mathrm{U}}_{\mathrm{A}}$ and $\overline{\mathrm{U}}_{\mathrm{B}}$ displacements of the wedge panels $\mathrm{A}$ and $\mathrm{B}$ :

Comparison between high-fidelity FEM and iFEM (Case IV) analyses.
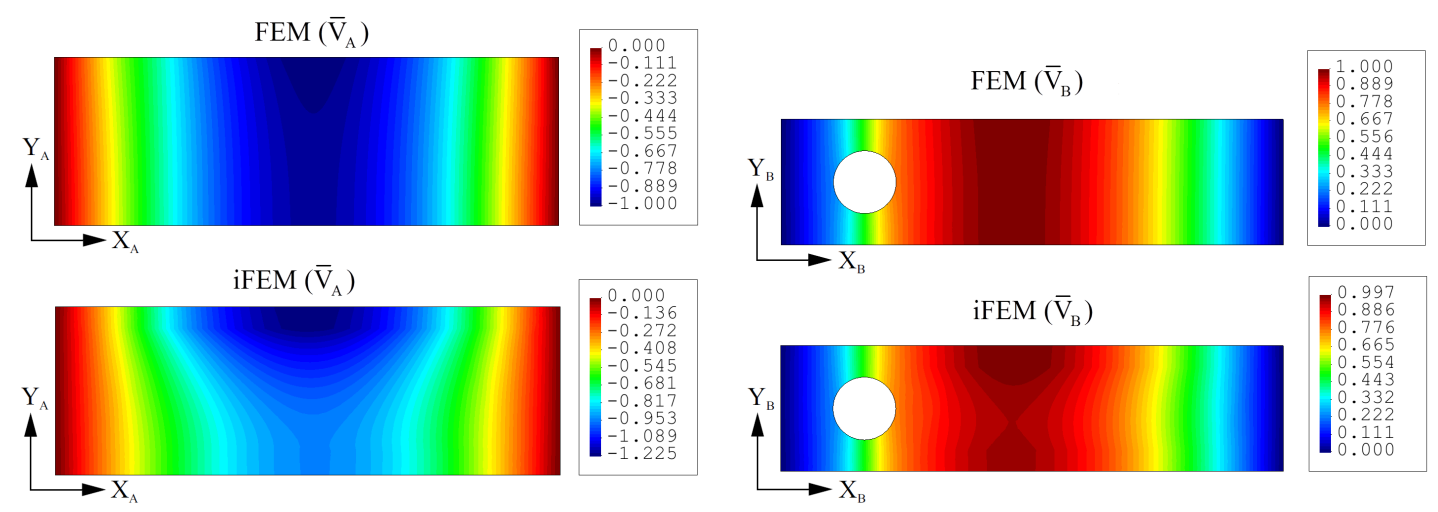

Figure 34. Contour plots of $\overline{\mathrm{V}}_{\mathrm{A}}$ and $\overline{\mathrm{V}}_{\mathrm{B}}$ displacements of the wedge panels $\mathrm{A}$ and B: Comparison between high-fidelity FEM and iFEM (Case IV) analyses.
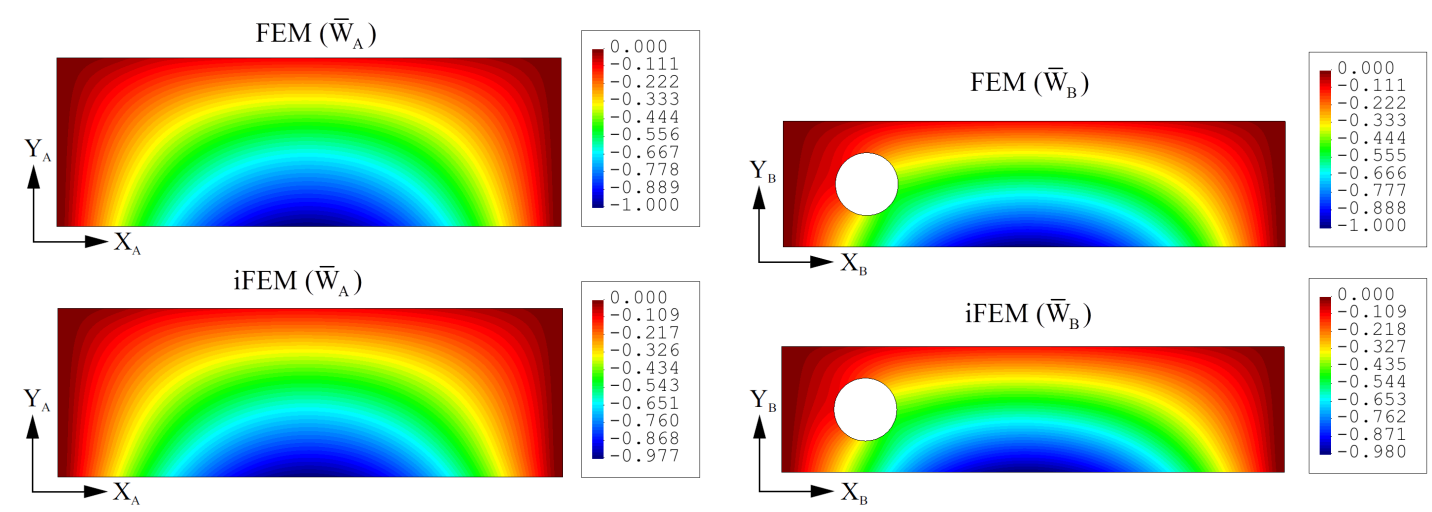

Figure 35. Contour plots of $\overline{\mathrm{W}}_{\mathrm{A}}$ and $\overline{\mathrm{W}}_{\mathrm{B}}$ displacements of the wedge panels $\mathrm{A}$ and $\mathrm{B}$ : Comparison between high-fidelity FEM and iFEM (Case IV) analyses. 

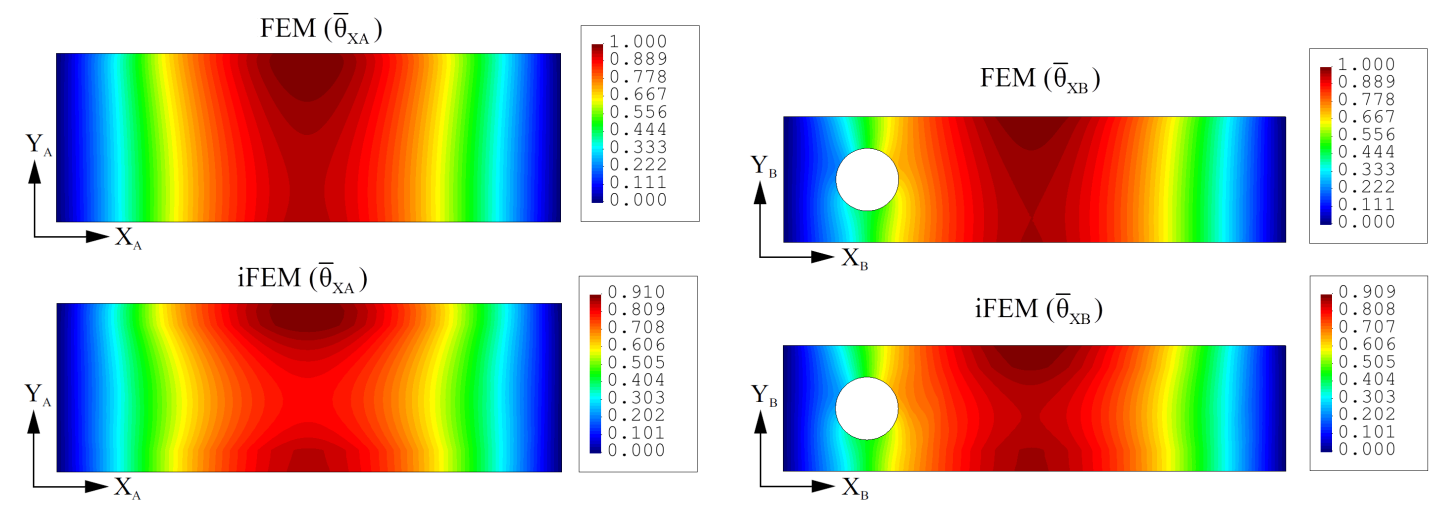

Figure 36. Contour plots of $\bar{\theta}_{\mathrm{XA}}$ and $\bar{\theta}_{\mathrm{XB}}$ bending rotations of the wedge panels $\mathrm{A}$ and B: Comparison between high-fidelity FEM and iFEM (Case IV) analyses.
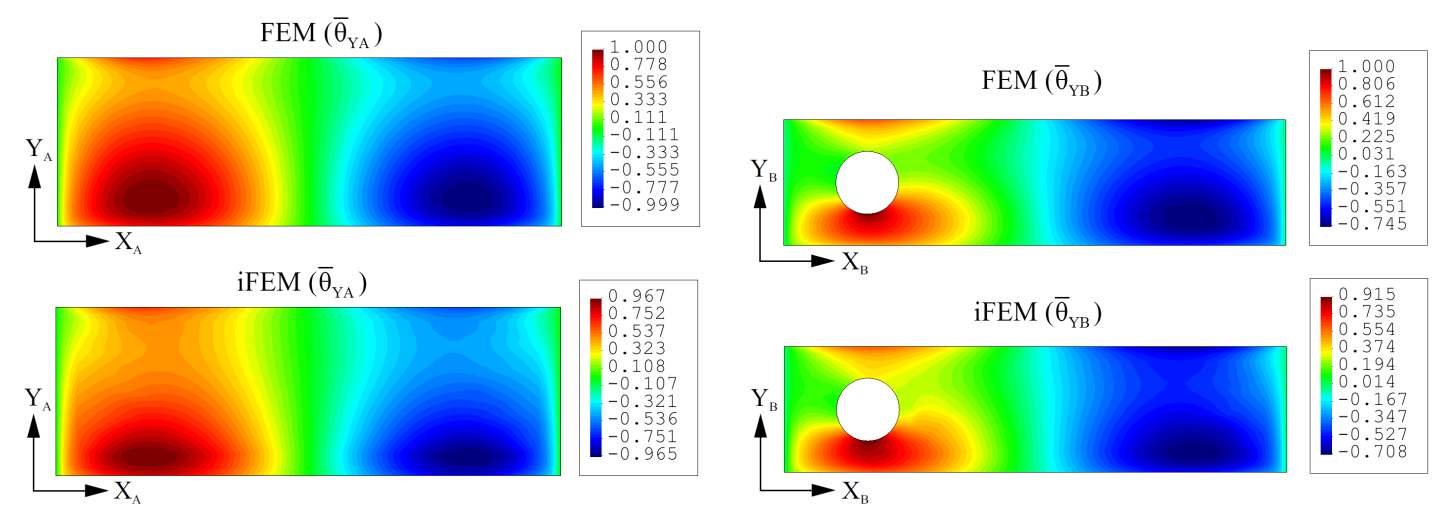

Figure 37. Contour plots of $\bar{\theta}_{\mathrm{YA}}$ and $\bar{\theta}_{\mathrm{YB}}$ bending rotations of the wedge panels $\mathrm{A}$ and B: Comparison between high-fidelity FEM and iFEM (Case IV) analyses.
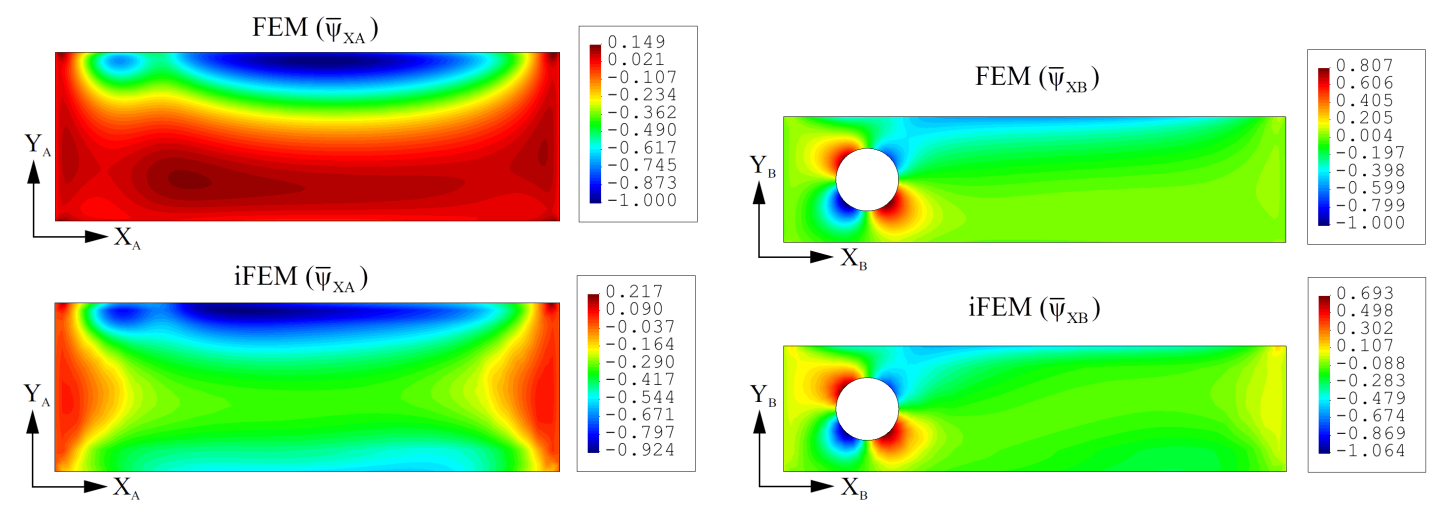

Figure 38. Contour plots of $\bar{\psi}_{\mathrm{XA}}$ and $\bar{\psi}_{\mathrm{XB}}$ zigzag rotations of the wedge panels A and B: Comparison between high-fidelity FEM and iFEM (Case IV) analyses. 

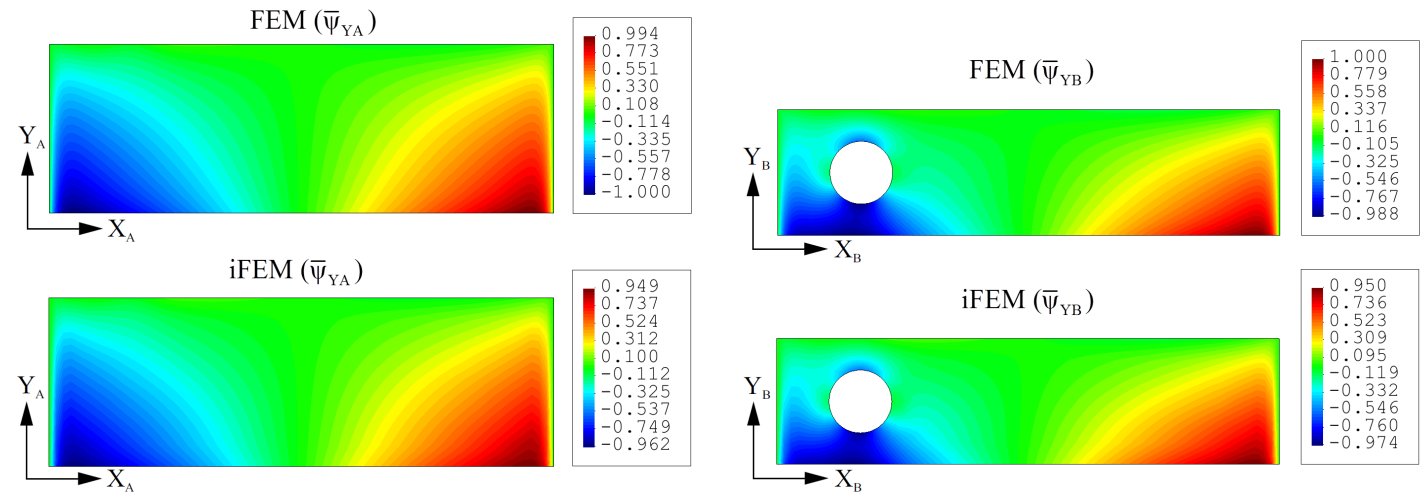

Figure 39. Contour plots of $\bar{\psi}_{\mathrm{YA}}$ and $\bar{\psi}_{\mathrm{YB}}$ zigzag rotations of the wedge panels A and B: Comparison between high-fidelity FEM and iFEM (Case IV) analyses.
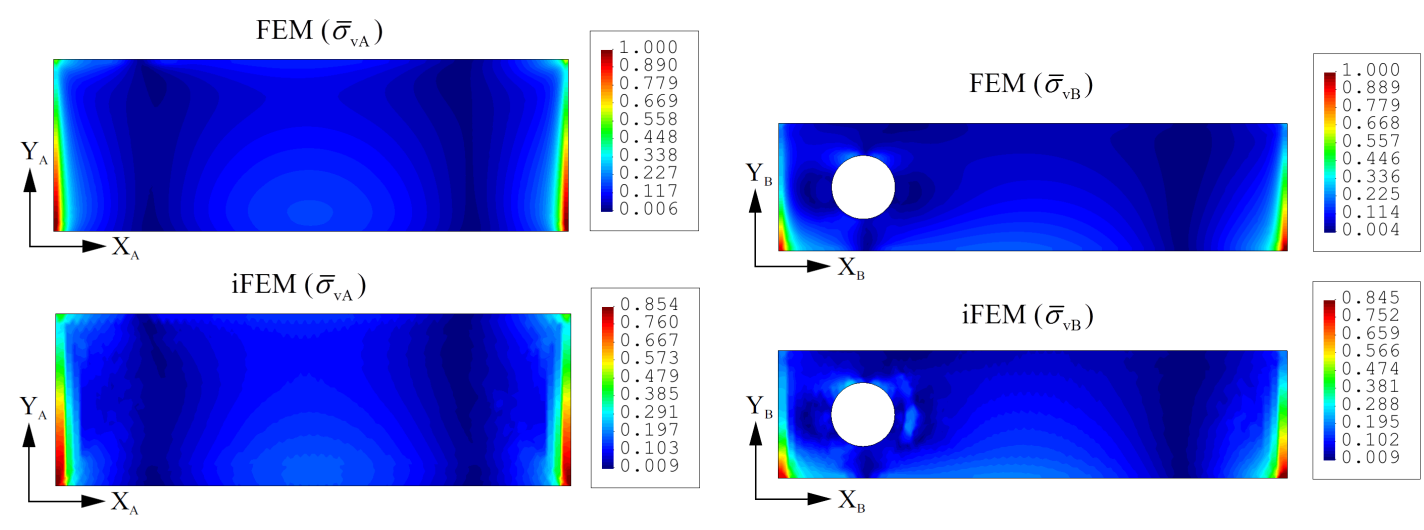

Figure 40. Contour plots of $\bar{\sigma}_{\mathrm{vA}}$ and $\bar{\sigma}_{\mathrm{vB}}$ von Mises stresses at thickness coordinates

$\mathrm{Z}_{\mathrm{A}} / \mathrm{h}=-1$ and $\mathrm{Z}_{\mathrm{B}} / \mathrm{h}=1$ of wedge panels $\mathrm{A}$ and $\mathrm{B}$, respectively: Comparison between high-fidelity FEM and iFEM (Case IV) analyses.

Furthermore, in Figures 41-46, variation of von Mises stress $\bar{\sigma}_{\mathrm{vA}}$ along $\mathrm{L}_{1}$ and $\mathrm{L}_{2}$ at the thickness coordinate $\mathrm{Z}_{\mathrm{A}} / \mathrm{h}=-1$ of wedge panel $\mathrm{A}$ is compared between iFEM (Cases I, III, and V) and reference FEM solutions, respectively. These results prove the following three observations: (1) the improved iFEM formulation predicts von Mises stresses that are comparably accurate to those of the reference solutions, (2) the i3-RZT element formulation requires higher fidelity discretization to calculate high strain gradients to obtain improved stresses, and finally (3) an i3-RZT model with relatively very sparse measured strain data can predict von Mises stresses that are as accurate as those obtained using an i3-RZT model in which all elements have strainsensor data. 


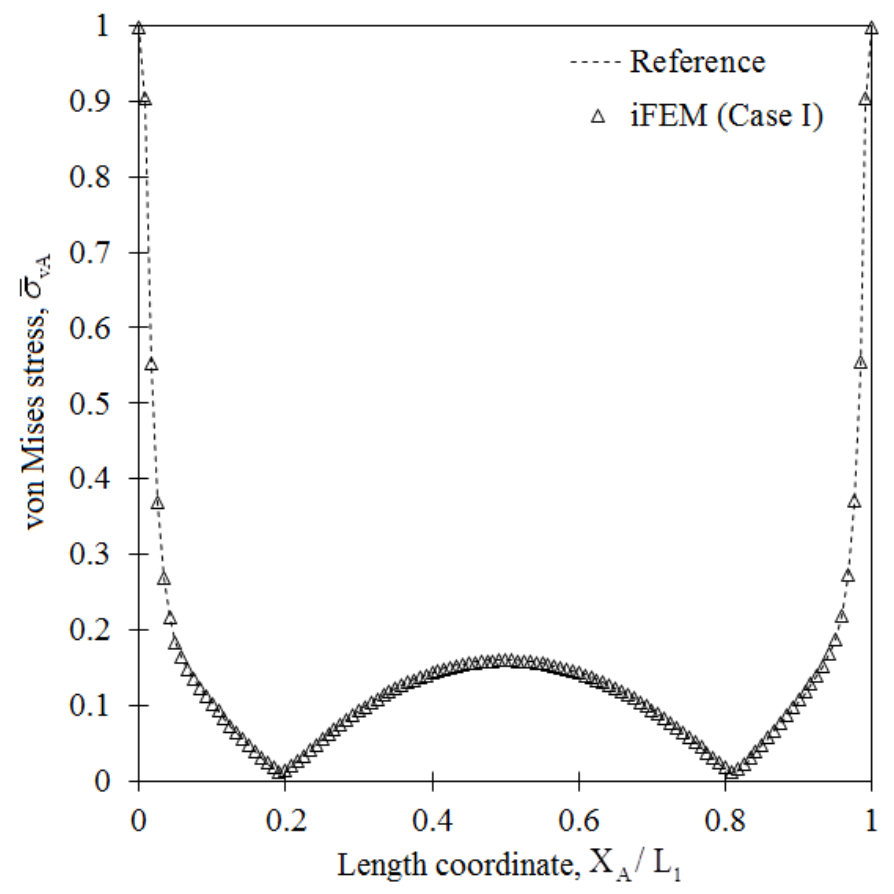

Figure 41. The von Mises stress $\bar{\sigma}_{\mathrm{vA}}$ variation along length $\mathrm{L}_{1}$ at $\mathrm{Z}_{\mathrm{A}} / \mathrm{h}=-1$ :

Comparison between high-fidelity FEM and iFEM (Case I) analyses.

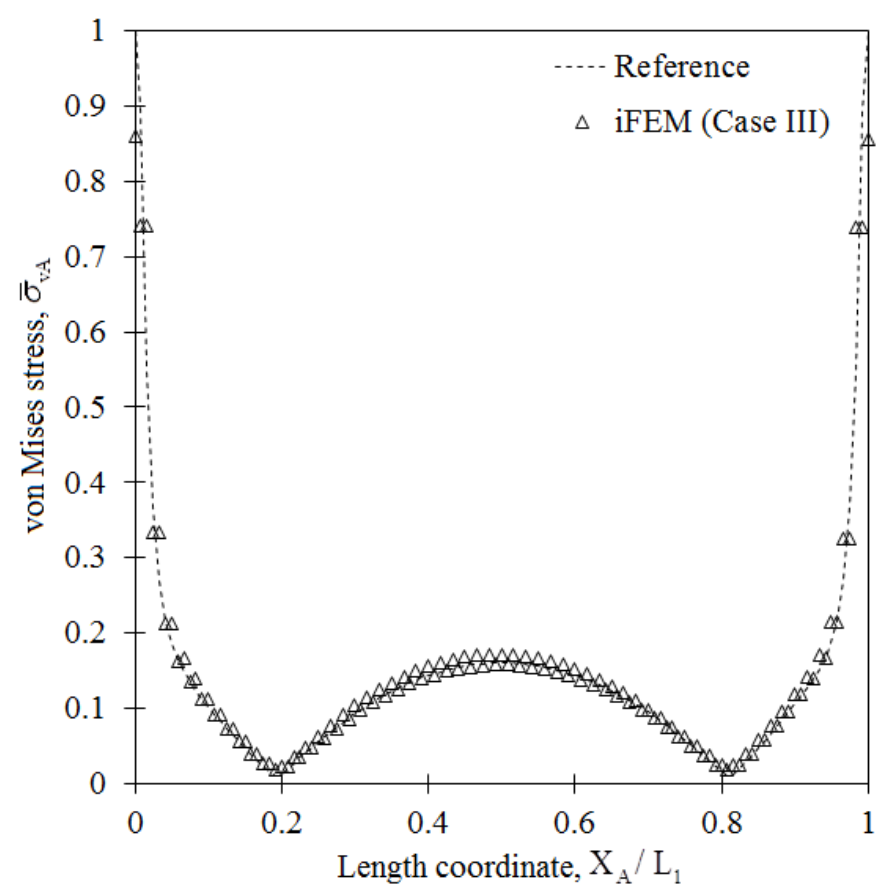

Figure 42. The von Mises stress $\bar{\sigma}_{\mathrm{vA}}$ variation along length $\mathrm{L}_{1}$ at $\mathrm{Z}_{\mathrm{A}} / \mathrm{h}=-1$ :

Comparison between high-fidelity FEM and iFEM (Case III) analyses. 


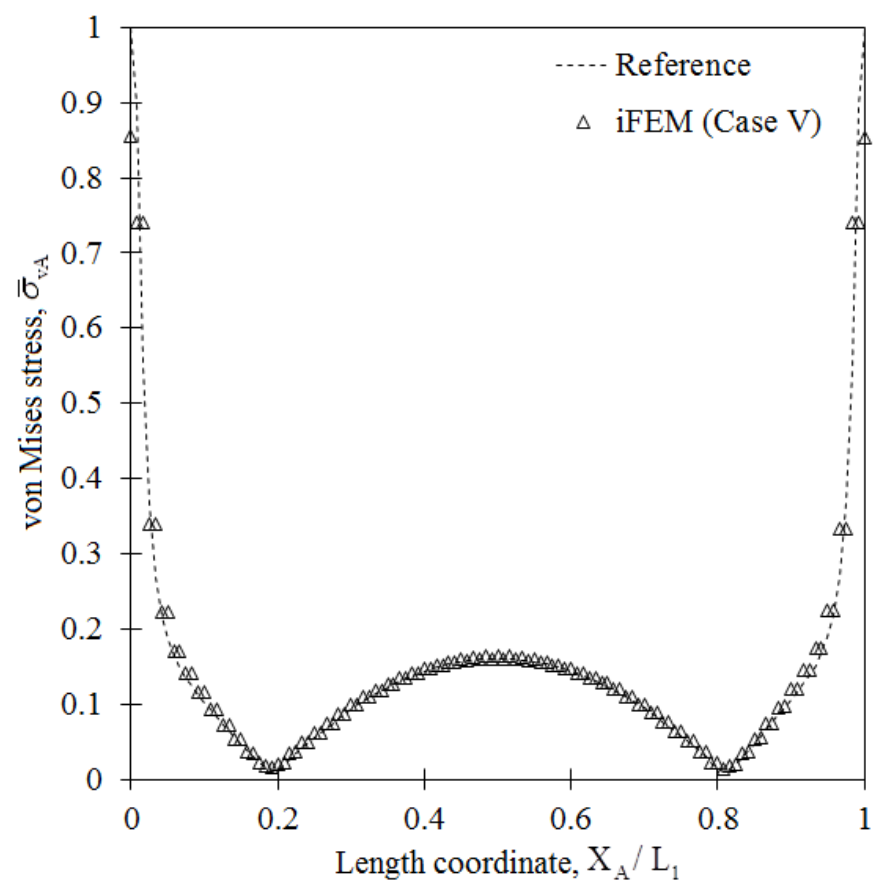

Figure 43. The von Mises stress $\bar{\sigma}_{\mathrm{vA}}$ variation along length $\mathrm{L}_{1}$ at $\mathrm{Z}_{\mathrm{A}} / \mathrm{h}=-1$ :

Comparison between high-fidelity FEM and iFEM (Case V) analyses.

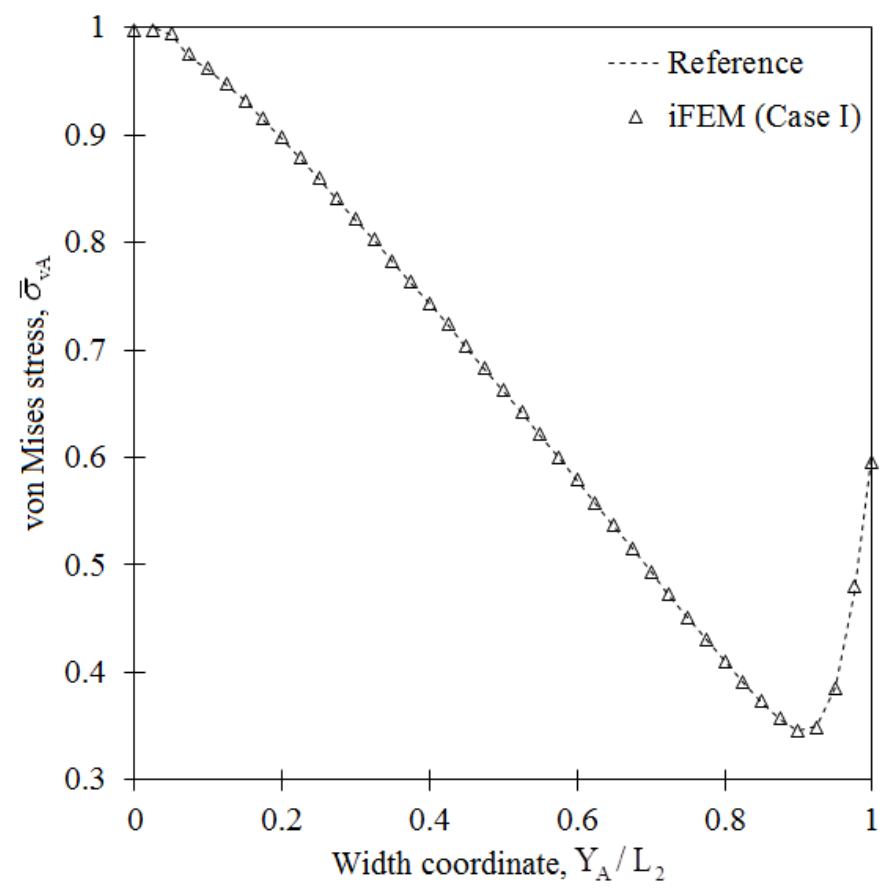

Figure 44. The von Mises stress $\bar{\sigma}_{\mathrm{vA}}$ variation along width $\mathrm{L}_{2}$ at $\mathrm{Z}_{\mathrm{A}} / \mathrm{h}=-1$ :

Comparison between high-fidelity FEM and iFEM (Case I) analyses. 


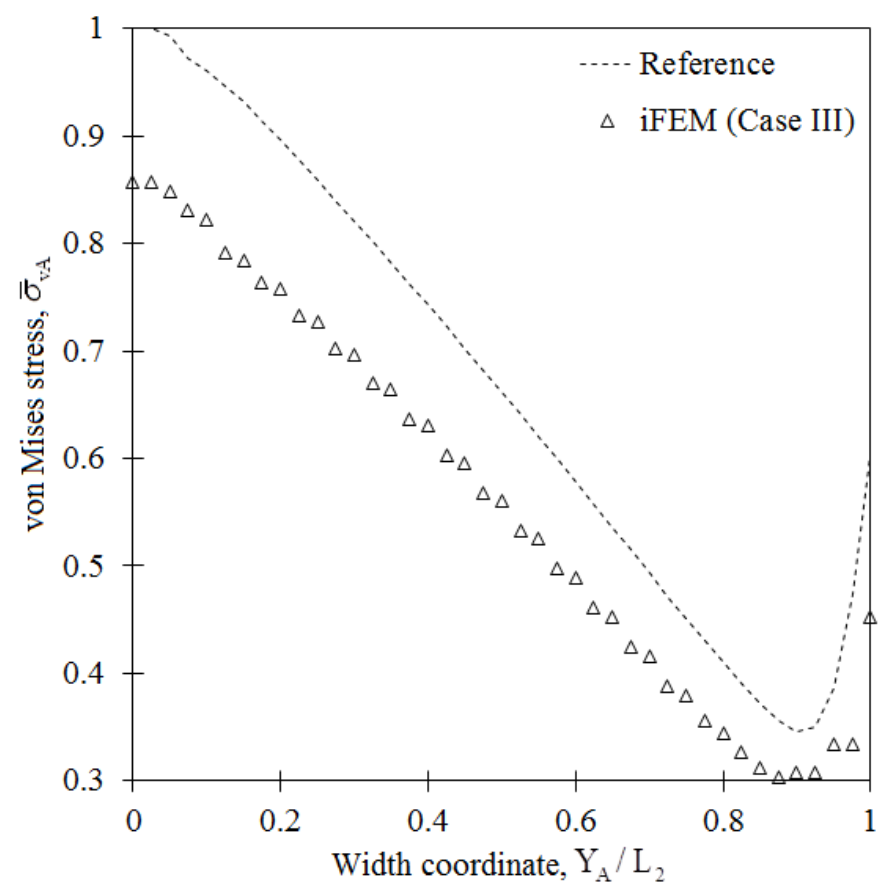

Figure 45. The von Mises stress $\bar{\sigma}_{\mathrm{vA}}$ variation along width $\mathrm{L}_{2}$ at $\mathrm{Z}_{\mathrm{A}} / \mathrm{h}=-1$ :

Comparison between high-fidelity FEM and iFEM (Case III) analyses.

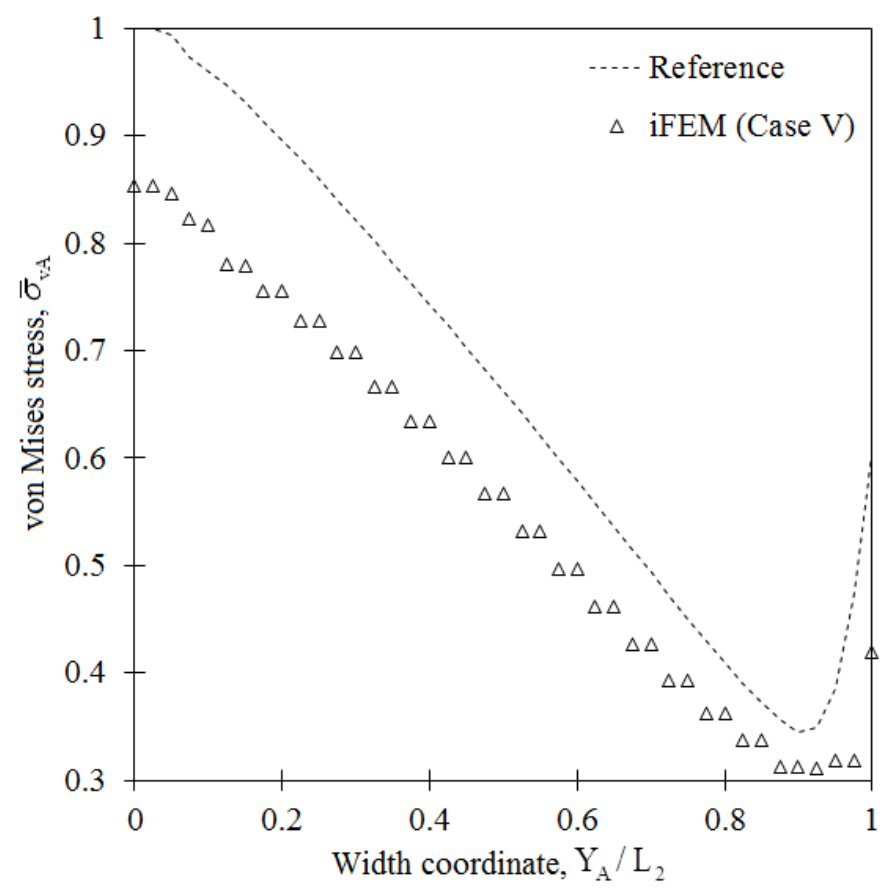

Figure 46. The von Mises stress $\bar{\sigma}_{\mathrm{vA}}$ variation along width $\mathrm{L}_{2}$ at $\mathrm{Z}_{\mathrm{A}} / \mathrm{h}=-1$ :

Comparison between high-fidelity FEM and iFEM (Case V) analyses. 


\section{Conclusion}

An improved iFEM formulation is presented to solve the inverse problem of shape and stress sensing of multilayered composite and sandwich plates/shells that have strain sensors at discrete locations. The plate/shell kinematics are described using RZT plate theory. The formulation is based on minimization of a weighted-least-squares functional that accounts for the complete set of strain measures consistent with RZT plate theory. Based on the present iFEM methodology, laminated composite and sandwich plate/shell structures instrumented with relatively few strain gauges can be analyzed by utilizing weighting constants. One advantage of the present iFEM methodology is that it can be used for the analysis of thin and moderately thick plate and shell structures because the variational principle accommodates for full transverseshear deformation of RZT. Moreover, the formulation is suitable for $\mathrm{C}^{0}$-continuous discretization, enabling the development of robust inverse-shell elements for performing shape and stress sensing of complex engineering structures. A new threenode triangular inverse-shell element (i3-RZT) was developed based on the improved iFEM formulation. The i3-RZT element kinematic field accommodates quadratic interpolations that permit a robust drilling DOF implementation that has the advantage of avoiding singular solutions when modeling complex shell structures. The formulation is free from the membrane and shear-locking phenomena. Several numerical studies were performed and demonstrated the computational efficiency, high accuracy, and robustness of i3-RZT discretization with respect to the membrane, bending, and membrane-bending coupled structural responses. The practical utility of the iFEM/i3-RZT technology for application to engineering structures has been assessed using relatively low- and high-fidelity discretization strategies. The effects of sensor locations and number of sensors were also explored. It was demonstrated that even in the presence of relatively sparse strain data, sufficiently accurate reconstruction of deformed structural shapes and stresses can be achieved. Finally, the new iFEM/i3-RZT technology can be readily implemented in any general-purpose finite element software, providing a highly desirable and viable computational tool for real-time structural health monitoring of laminated-composite and sandwich structures. 


\section{Appendix A}

The coordinates of i3-RZT element nodes referred to the global coordinate system $(X, Y, Z)$ are given as

$\mathbf{X}_{i}=\left[\begin{array}{lll}X_{i} & Y_{i} & Z_{i}\end{array}\right]^{T}(i=1-3)$

Firstly, unit vector along local $x$-axis, $\mathbf{1}$, can be defined as a unit vector pointing out from node- 1 to node- 2 , that is

$$
\mathbf{I}=\frac{\mathbf{X}_{2}-\mathbf{X}_{1}}{\left\|\mathbf{X}_{2}-\mathbf{X}_{1}\right\|}
$$

Secondly, a unit vector pointing out from node- 1 to node-3 can be defined as

$$
\mathbf{A}=\frac{\mathbf{X}_{3}-\mathbf{X}_{1}}{\left\|\mathbf{X}_{3}-\mathbf{X}_{1}\right\|}
$$

Then, the cross product of these vectors $\mathbf{I}$ and $\mathbf{A}$ can establish a unit normal vector to the mid-plane triangle, $\mathbf{n}$, can be defined by

$$
\mathbf{n}=\mathbf{l} \times \mathbf{A}
$$

Finally, the unit vector along local $y$-axis, $\mathbf{p}$, can readily be computed from the cross product of the vectors $\mathbf{n}$ and $\mathbf{l}$ as

$\mathbf{p}=\mathbf{n} \times \mathbf{l}$

Each edge length $d_{i}$ of the mid-plane triangle and global coordinates of each edge's mid-point $\mathbf{c}_{i}$ can be calculated as

$$
\left.\begin{array}{l}
d_{i}=\left\|\mathbf{X}_{j}-\mathbf{X}_{i}\right\| \\
\mathbf{c}_{i}=\frac{\mathbf{X}_{j}+\mathbf{X}_{i}}{2}
\end{array}\right\}(i=1,2,3 ; j=2,3,1)
$$

Then, global coordinates of centroid of the mid-plane triangle can be defined as

$$
\mathbf{C}=\frac{\mathbf{c}_{1} d_{1}+\mathbf{c}_{2} d_{2}+\mathbf{c}_{3} d_{3}}{d_{1}+d_{2}+d_{3}}
$$


Using Eqs. (A.1-2), (A.5), and (A.7), local coordinates of the i3-RZT element nodes can be determined as

$\left.\begin{array}{l}x_{i}=\left(\mathbf{X}_{i}-\mathbf{C}\right) \cdot \mathbf{l} \\ y_{i}=\left(\mathbf{X}_{i}-\mathbf{C}\right) \cdot \mathbf{p}\end{array}\right\}(i=1-3)$

With the unit vectors $\mathbf{I}, \mathbf{p}$, and $\mathbf{n}$, the transformation matrix, $\mathbf{T}^{e}$, can be defined as

$$
\mathbf{T}^{e}=\left[\begin{array}{lllllllll}
\mathbf{T} & 0 & 0 & 0 & 0 & 0 & 0 & 0 & 0 \\
0 & \mathbf{T} & 0 & 0 & 0 & 0 & 0 & 0 & 0 \\
0 & 0 & \mathbf{T} & 0 & 0 & 0 & 0 & 0 & 0 \\
0 & 0 & 0 & \mathbf{T} & 0 & 0 & 0 & 0 & 0 \\
0 & 0 & 0 & 0 & \mathbf{T} & 0 & 0 & 0 & 0 \\
0 & 0 & 0 & 0 & 0 & \mathbf{T} & 0 & 0 & 0 \\
0 & 0 & 0 & 0 & 0 & 0 & \mathbf{T} & 0 & 0 \\
0 & 0 & 0 & 0 & 0 & 0 & 0 & \mathbf{T} & 0 \\
0 & 0 & 0 & 0 & 0 & 0 & 0 & 0 & \mathbf{T}
\end{array}\right]
$$

with

$$
\mathbf{T}=\left[\begin{array}{lll}
\mathbf{l}^{T} & \mathbf{p}^{T} & \mathbf{n}^{T}
\end{array}\right]^{T}
$$

where $\mathbf{T}$ is the stress transformation matrix from the local to the global coordinate system. 


\section{Appendix B}

The matrices $\mathbf{B}_{\alpha}(\alpha=1-13)$ used in Eqs. (28a-e) contain derivatives of the shape functions and can be expressed as

$\mathbf{B}_{\alpha}=\left[\begin{array}{lll}\mathbf{B}_{\alpha}^{1} & \mathbf{B}_{\alpha}^{2} & \mathbf{B}_{\alpha}^{3}\end{array}\right] \quad(\alpha=1-13)$

with

$$
\begin{aligned}
& \mathbf{B}_{1}^{i}=\left[\begin{array}{lllllllll}
N_{i, x} & 0 & 0 & 0 & 0 & L_{i, x} & 0 & 0 & 0
\end{array}\right] \\
& \left.\begin{array}{rl}
\mathbf{B}_{2}^{i} & =\left[\begin{array}{lllllllll}
0 & N_{i, y} & 0 & 0 & 0 & M_{i, y} & 0 & 0 & 0
\end{array}\right] \\
\mathbf{B}_{3}^{i} & =\left[\begin{array}{lllllllll}
N_{i, y} & N_{i, x} & 0 & 0 & 0 & L_{i, y}+M_{i, x} & 0 & 0 & 0
\end{array}\right]
\end{array}\right\}(i=1-3) \\
& \mathbf{B}_{4}^{i}=\left[\begin{array}{lllllllll}
0 & 0 & 0 & 0 & N_{i, x} & 0 & 0 & 0 & 0
\end{array}\right] \\
& \mathbf{B}_{5}^{i}=\left[\begin{array}{lllllllll}
0 & 0 & 0 & -N_{i, y} & 0 & 0 & 0 & 0 & 0
\end{array}\right] \quad(i=1-3) \\
& \left.\mathbf{B}_{6}^{i}=\left[\begin{array}{lllllllll}
0 & 0 & 0 & -N_{i, x} & N_{i, y} & 0 & 0 & 0 & 0
\end{array}\right]\right)
\end{aligned}
$$

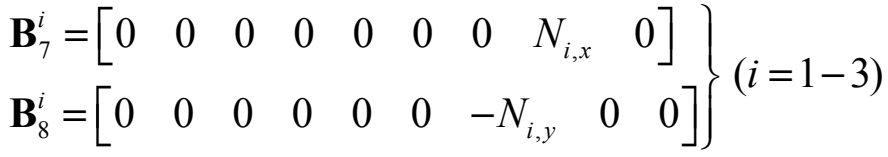

$$
\begin{aligned}
& \mathbf{B}_{9}^{i}=\left[\begin{array}{ccccccccc}
0 & 0 & 0 & 0 & 0 & 0 & 0 & N_{i, y} & 0 \\
0 & 0 & 0 & 0 & 0 & 0 & -N_{i, x} & 0 & 0
\end{array}\right](i=1-3)
\end{aligned}
$$

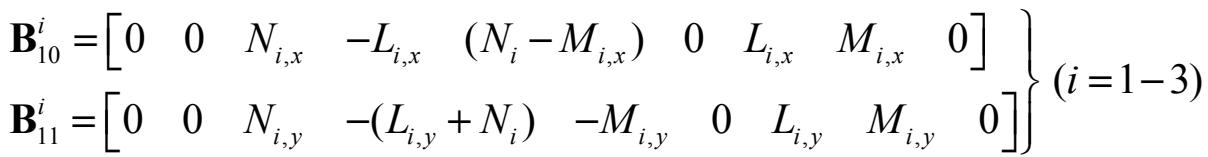

$$
\begin{aligned}
& \left.\begin{array}{l}
\mathbf{B}_{12}^{i}=\left[\begin{array}{lllllllll}
0 & 0 & N_{i, x} & -L_{i, x} & \left(N_{i}-M_{i, x}\right) & 0 & L_{i, x} & \left(M_{i, x}-N_{i}\right) & 0
\end{array}\right] \\
\mathbf{B}_{13}^{i}=\left[\begin{array}{lllllllll}
0 & 0 & N_{i, y} & -\left(L_{i, y}+N_{i}\right) & -M_{i, y} & 0 & \left(L_{i, y}+N_{i}\right) & M_{i, y} & 0
\end{array}\right]
\end{array}\right\}(i=1-3)
\end{aligned}
$$

where, ( $)_{, x} \equiv \frac{\partial()}{\partial x}$ and ( $)_{, y} \equiv \frac{\partial()}{\partial y}$ denote the partial derivative with respect to in-plane coordinates $x$ and $y$, respectively. Note that $N_{i}, L_{i}$, and $M_{i}(i=1-3)$ are the shape functions of the i3-RZT element, which have been explicitly given by Eqs. (27a-b). 


\section{Appendix C}

After the left-hand-side matrix $\boldsymbol{\Gamma}^{e}$ is constructed using the Eq. (31), the element equation, $\boldsymbol{\Gamma}^{e} \mathbf{u}^{e}=\boldsymbol{\varepsilon}^{e}$, can be arranged in the following form

$$
\left[\begin{array}{cc}
\boldsymbol{\Gamma}_{v} & \mathbf{0} \\
\mathbf{0} & \mathbf{0}
\end{array}\right]\left[\begin{array}{c}
\mathbf{v} \\
\boldsymbol{\psi}_{z}
\end{array}\right]=\left[\begin{array}{c}
\mathbf{f}_{v} \\
\mathbf{0}
\end{array}\right]
$$

with

$\mathbf{v}=\left[\begin{array}{lll}\mathbf{v}_{1} & \mathbf{v}_{2} & \mathbf{v}_{3}\end{array}\right]^{T}, \mathbf{v}_{i}=\left[\begin{array}{llllllll}u_{i} & v_{i} & w_{i} & \theta_{x i} & \theta_{y i} & \theta_{z i} & \psi_{x i} & \psi_{y i}\end{array}\right]^{T}(i=1-3)$

and

$\boldsymbol{\psi}_{z}=\left[\begin{array}{lll}\psi_{z 1} & \psi_{z 2} & \psi_{z 3}\end{array}\right]^{T}$

where the $\boldsymbol{\psi}_{z}$ and $\mathbf{v}$ vectors contain artificial zigzag rotations and all other DOF of the i3-RZT element, respectively. An artificial contribution matrix $\boldsymbol{\Gamma}_{\psi z}$ that corresponds to the $\boldsymbol{\psi}_{z}$ can be constructed as follows

$\boldsymbol{\Gamma}_{\psi z}=\left[\begin{array}{ccc}k_{\psi z}^{1} & 0 & 0 \\ 0 & k_{\psi z}^{2} & 0 \\ 0 & 0 & k_{\psi z}^{3}\end{array}\right]$

with

$k_{\psi z}^{i}=\lambda \times \min \left(k_{\psi x}^{i}, k_{\psi y}^{i}\right) \quad(i=1,2,3)$

where the constant $\lambda$ is a small number, e.g., $\lambda=10^{-5}$, and the coefficients $k_{\psi x}^{i}, k_{\psi y}^{i}(i=1,2,3)$ are diagonal terms of the $\boldsymbol{\Gamma}_{v}$ matrix corresponding to zigzagamplitude DOF, $\left(\psi_{x i}, \psi_{y i}\right)(i=1-3)$. To avoid singular solutions, $\boldsymbol{\Gamma}_{\psi z}$ must be added to the $\Gamma^{e}$ matrix, and the element equations defined in Eq. (C.1) can be rewritten in the following final form as

$$
\left[\begin{array}{cc}
\boldsymbol{\Gamma}_{v} & \mathbf{0} \\
\mathbf{0} & \boldsymbol{\Gamma}_{\psi z}
\end{array}\right]\left[\begin{array}{c}
\mathbf{v} \\
\boldsymbol{\psi}_{z}
\end{array}\right]=\left[\begin{array}{c}
\mathbf{f}_{v} \\
\mathbf{0}
\end{array}\right]
$$




\section{References}

[1] Glisic, B., and Inaudi, D. (2007). Fibre Optic Methods for Structural Health Monitoring. John Wiley and Sons, England.

[2] Herrmann, A.S., Zahlen, P.C., and Zuardy, I. (2005). Sandwich structures technology in commercial aviation. In O.T. Thomsen et al. (Eds.), Sandwich Structures 7: Advancing with Sandwich Structures and Materials, pp. 13-26. Springer, Netherlands.

[3] Lolive, E., Casari, P., and Davies, P. (2005). Loading rate effects on foam cores for marine sandwich structures. In O.T. Thomsen et al. (Eds.), Sandwich structures 7: Advancing with Sandwich Structures and Materials, pp. 895-903. Springer, Netherlands.

[4] Berggreen, C., Branner, K., Jensen, J.F., and Schultz, J.P. (2007). Application and analysis of sandwich elements in the primary structure of large wind turbine blades. Journal of Sandwich Structures and Materials, 9(6): 525-552.

[5] Zou, Y., Tong, L.P.S.G., and Steven, G.P. (2000). Vibration-based modeldependent damage (delamination) identification and health monitoring for composite structures - a review. Journal of Sound and Vibration, 230(2): 357378.

[6] McCartney, L.N. (1987). Mechanics of matrix cracking in brittle-matrix fibrereinforced composites. Proceedings of the Royal Society of London A: Mathematical, Physical and Engineering Sciences, 409(1837): 329-350.

[7] Vadakke, V., and Carlsson, L.A. (2004). Experimental investigation of compression failure of sandwich specimens with face/core debond. Composites Part B: Engineering, 35(6): 583-590.

[8] Bray, D.E., and McBride, D. (1992). Nondestructive Testing Techniques. John Wiley and Sons, New York.

[9] Yin, W., Fu, T., Liu, J., and Leng, J. (2009). Structural shape sensing for variable camber wing using FBG sensors. Proceedings of the International Society for Optical Engineering, 7292: 72921H. 
[10] Lee, J.R., Ryu, C.Y., Koo, B.Y., Kang, S.G., Hong, C.S., and Kim, C.G. (2003). In-flight health monitoring of a subscale wing using a fiber Bragg grating sensor system. Smart Materials and Structures, 12(1): 147-155.

[11] Dawood, T.A., Shenoi, R.A., and Sahin, M. (2007). A procedure to embed fibre Bragg grating strain sensors into GFRP sandwich structures. Composites Part A: Applied Science and Manufacturing, 38(1): 217-226.

[12] Gherlone, M., Cerracchio, P., Mattone, M., Di Sciuva, M., and Tessler, A. (2012). Shape sensing of 3D frame structures using an inverse finite element method. International Journal of Solids and Structures, 49(22): 3100-3112.

[13] Tikhonov, A.N., and Arsenin, V.Y. (1977). Solutions of Ill-Posed Problems. Winston and Sons, Washington, DC.

[14] Maniatty, A.M., and Zabaras, N.J. (1989). Method for solving inverse elastoviscoplastic problems. Journal of Engineering Mechanics, 115(10): 2216-2231

[15] Maniatty, A.M., and Zabaras, N.J. (1994). Investigation of regularization parameters and error estimating in inverse elasticity problems. International Journal for Numerical Methods in Engineering, 37(6): 1039-1052.

[16] Maniatty, A.M., Zabaras, N.J., and Stelson, K. (1989). Finite element analysis of some inverse elasticity problems. Journal of Engineering Mechanics, 115(6): 1303-1317.

[17] Schnur, D.S., and Zabaras, N. (1990). Finite element solution of twodimensional inverse elastic problems using spatial smoothing. International Journal for Numerical Methods in Engineering, 30(1): 57-75.

[18] Davis, M.A., Kersey, A.D., Sirkis, J., and Friebele, E.J. (1996). Shape and vibration mode sensing using a fiber optic Bragg grating array. Smart Materials and Structures, 5(6): 759-765.

[19] Ko, W.L., Richards, W.L., and Fleischer, V.T. (2009). Applications of Ko displacement theory to the deformed shape predictions of the doubly-tapered Ikhana Wing. NASA/TP-2009-214652. 
[20] Glaser, R., Caccese, V., and Shahinpoor, M. (2012). Shape monitoring of a beam structure from measured strain or curvature. Experimental Mechanics, 52(6): 591-606.

[21] Chierichetti, M. (2014). Load and response identification for a nonlinear flexible structure subject to harmonic loads. Journal of Computational and Nonlinear Dynamics, 9(1): 011009.

[22] Jones, R.T., Bellemore, D.G., Berkoff, T.A., Sirkis, J.S., Davis, M.A., Putnam, M.A., and Kersey, A.D. (1998). Determination of cantilever plate shapes using wavelength division multiplexed fiber Bragg grating sensors and a leastsquares strain-fitting algorithm. Smart Materials and Structures, 7(2): 178188.

[23] Bogert, P.B., Haugse, E.D., and Gehrki, R.E. (2003). Structural shape identification from experimental strains using a modal transformation technique. Proceedings of 44th AIAA/ASME/ASCE/AHS Structures, Structural Dynamics and Materials Conference. Norfolk, VA.

[24] Rapp, S., Kang, L.H., Han, J.H., Mueller, U.C., and Baier, H. (2009). Displacement field estimation for a two-dimensional structure using fibre Bragg grating sensors. Smart Materials and Structures, 18(2): 025006.

[25] Nishio, M., Mizutani, T., Takeda, N. (2010). Structural shape reconstruction with consideration of the reliability of distributed strain data from a Brillouinscattering-based optical fiber sensor. Smart Materials and Structures, 19(3): 035011.

[26] Tessler, A., and Spangler, J.L. (2003). A variational principal for reconstruction of elastic deformation of shear deformable plates and shells. NASA TM-2003-212445.

[27] Tessler, A., and Spangler, J.L. (2005). A least-squares variational method for full-field reconstruction of elastic deformations in shear-deformable plates and shells. Computer Methods in Applied Mechanics and Engineering, 194(2): 327-339. 
[28] Tessler, A., and Spangler, J.L. (2004). Inverse FEM for full-field reconstruction of elastic deformations in shear deformable plates and shells. Proceedings of 2nd European Workshop on Structural Health Monitoring. Munich, Germany.

[29] Kefal, A., Oterkus, E., Tessler, A., and Spangler, J.L. (2016). A quadrilateral inverse-shell element with drilling degrees of freedom for shape sensing and structural health monitoring. Engineering Science and Technology, an International Journal, 19: 1299-1313.

[30] Cerracchio, P., Gherlone, M., Mattone, M., Di Sciuva, M., and Tessler, A. (2010). Shape sensing of three-dimensional frame structures using the inverse finite element method. Proceedings of 5th European Workshop on Structural Health Monitoring. Sorrento, Italy.

[31] Gherlone, M., Cerracchio, P., Mattone, M., Di Sciuva, M., and Tessler, A. (2011). Dynamic shape reconstruction of three-dimensional frame structures using the inverse finite element method. NASA/TP-2011-217315.

[32] Gherlone, M., Cerracchio, P., Mattone, M., Di Sciuva, M., and Tessler, A. (2014). An inverse finite element method for beam shape sensing: theoretical framework and experimental validation. Smart Materials and Structures, 23(4), 045027.

[33] Tessler, A. (2007). Structural analysis methods for structural health management of future aerospace vehicles. Key Engineering Materials, 347: 57 66.

[34] Tessler, A., Spangler, J.L., Gherlone M., Mattone M., and Di Sciuva, M. (2012). Deformed shape and stress reconstruction in plate and shell structures undergoing large displacements: application of inverse finite element method using fiber Bragg grating strains. 10th World Congress on Computational Mechanics, Sao Paulo, Brazil. Retrieved from https://ifem.larc.nasa.gov/publi cations/2012-WCCM-Brazil-Tessler.pdf. 
[35] Cerracchio, P., Gherlone, M., and Tessler, A. (2015). Real-time displacement monitoring of a composite stiffened panel subjected to mechanical and thermal loads. Meccanica, 50(10): 2487-2496.

[36] Kefal, A., and Oterkus, E. (2015). Structural health monitoring of marine structures by using inverse finite element method. In C. Guedes Soares and R.A. Shenoi (Eds.), Analysis and Design of Marine Structures V, pp. 341-349, Taylor and Francis Group, London.

[37] Kefal, A., and Oterkus, E. (2016). Displacement and stress monitoring of a chemical tanker based on inverse finite element method. Ocean Engineering, 112: $33-46$.

[38] Kefal, A., and Oterkus, E. (2016). Displacement and stress monitoring of a Panamax containership using inverse finite element method. Ocean Engineering, 119: 16-29.

[39] Stoesz, C.W. (2013). Method for Analyzing Strain Data. US 8,515,675 B2.

[40] Reissner, E. (1945). The effect of transverse shear deformation on the bending of elastic plates. Journal of Applied Mechanics (American Society of Mechanical Engineers: ASME), 12: 69-77.

[41] Mindlin, R.D. (1951). Influence of rotatory inertia and shear deformation on flexural motions of isotropic elasticplates. Journal of Applied Mechanics (American Society of Mechanical Engineers: ASME), 18: 31-38.

[42] Tessler, A., Di Sciuva, M., and Gherlone, M. (2009). A refined zigzag beam theory for composite and sandwich beams. Journal of Composite Materials, 43: 1051-1081.

[43] Tessler, A., Di Sciuva, M., and Gherlone, M. (2010). A consistent refinement of first-order shear deformation theory for laminated composite and sandwich plates using improved zigzag kinematics. Journal of Mechanics of Materials and Structures, 5(2): 341-367. 
[44] Cerracchio, P., Gherlone, M., Di Sciuva, M., and Tessler, A. (2013). Shape and stress sensing of multilayered composite and sandwich structures using an inverse finite element method. Proceedings of $V$ International Conference on Computational Methods for Coupled Problems in Science and Engineering. Ibiza, Spain.

[45] Cerracchio, P., Gherlone, M., Di Sciuva, M., and Tessler, A. (2015). A novel approach for displacement and stress monitoring of sandwich structures based on the inverse finite element method. Composite Structures, 127: 69-76.

[46] Tessler, A., Spangler, J.L., Gherlone, M., Mattone, M., and Di Sciuva, M. (2011). Real-time characterization of aerospace structures using onboard strain measurement technologies and inverse finite element method. Proceedings of the 8th International Workshop on Structural Health Monitoring. Stanford, CA.

[47] Lancaster, P., and Salkauskas, K. (1986). Curve and Surface Fitting: An Introduction. Academic Press, London.

[48] Tessler, A., Riggs, H.R., Freese, C.E., and Cook, G.M. (1998). An improved variational method for finite element stress recovery and a posteriori error estimation. Computer Methods in Applied Mechanics and Engineering, 155(1): 15-30.

[49] Tessler, A., Riggs, H.R., and Dambach, M. (1999). A novel four-node quadrilateral smoothing element for stress enhancement and error estimation. International Journal for Numerical Methods in Engineering, 44(10): 15271541.

[50] Tessler, A., and Dong, S.B. (1981). On a hierarchy of conforming Timoshenko beam elements. Computers and Structures, 14(3): 335-344.

[51] Tessler, A. (2000). Comparison of interdependent interpolations for membrane and bending kinematics in shear-deformable shell elements. Proceedings of International Conference on Computational Engineering and Sciences. Los Angeles, CA. 
[52] Tessler, A., and Hughes, T.J.R. (1985). A three-node Mindlin plate element with improved transverse shear. Computer Methods in Applied Mechanics and Engineering, 50(1): 71-101.

[53] Versino, D., Gherlone, M., Mattone, M., Di Sciuva, M., and Tessler, A. (2013). $\mathrm{C}^{0}$ triangular elements based on the Refined Zigzag Theory for multilayer composite and sandwich plates. Composites Part B: Engineering, 44(1): 218230.

[54] Pagano, N.J. (1969). Exact Solutions for Composite Laminates in Cylindrical Bending. Journal of Composite Materials, 3(3): 398-411.

[55] Di Sciuva, M. (1984). A refinement of the transverse shear deformation theory for multilayered orthotropic plates. L'aerotecnica Missili e Spazio, 62: 84-92.

[56] Kefal, A., and Oterkus, E. (2017). Shape sensing of aerospace structures by coupling of isogeometric analysis and inverse finite element method. Proceedings of the 58th AIAA/ASCE/AHS/ASC Structures, Structural Dynamics and Materials Conference. Grapevine, TX. 